\title{
BOREHOLE P- AND S-WAVE VELOCITY AT THIRTEEN STATIONS IN SOUTHERN CALIFORNIA
}

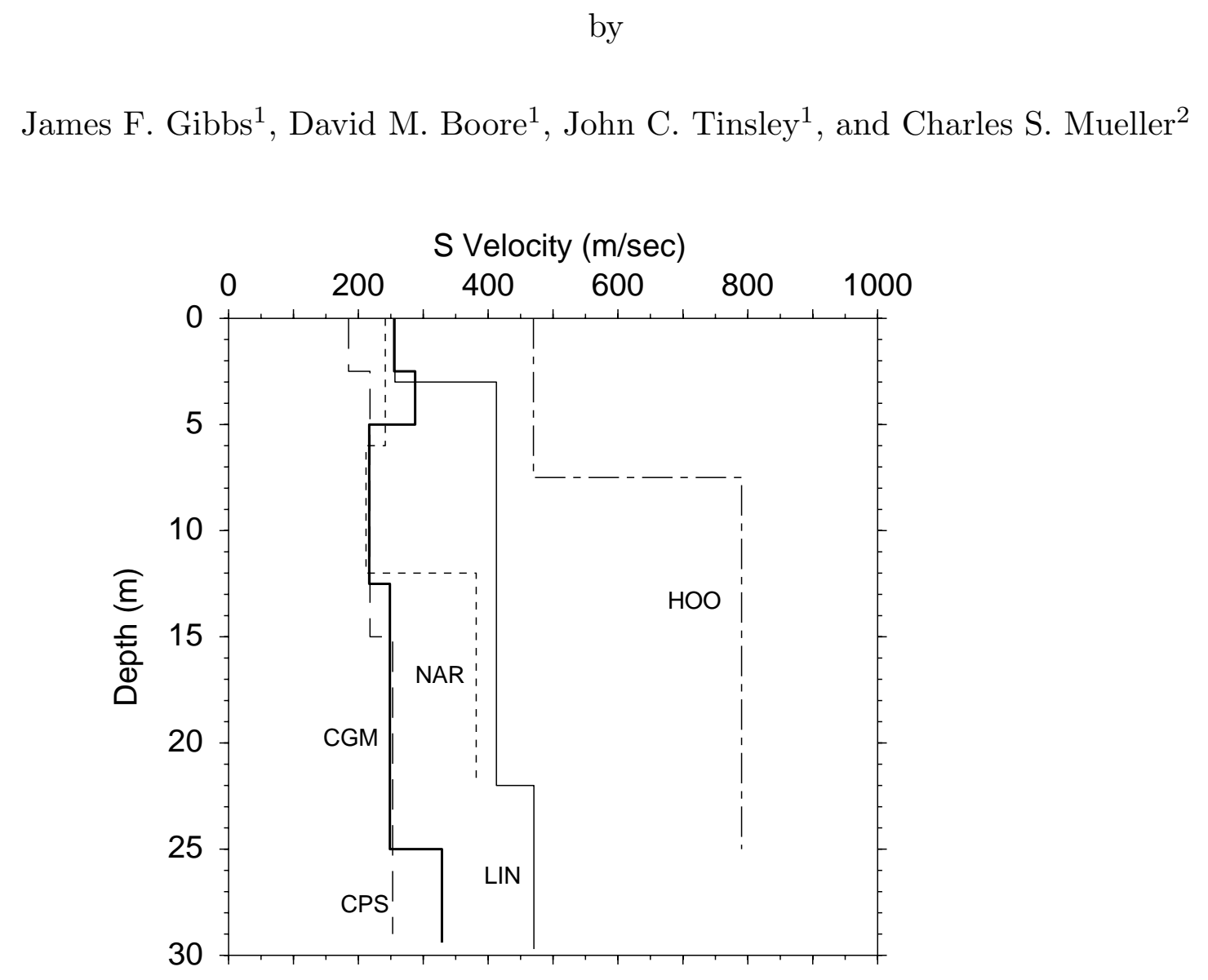

\section{U.S. Geological Survey Open-File Report OF 01 - 506}

This report is preliminary and has not been reviewed for conformity with U.S. Geological Survey editorial standards or with the North American Stratigraphic Code. Any use of trade, product, or firm names is for descriptive purposes only and does not imply endorsement by the U.S. Government.

\footnotetext{
${ }^{1}$ Menlo Park, CA 94025

${ }^{2}$ Denver, CO 80225
} 


\section{TABLE OF CONTENTS}

Introduction . . . . . . . . . . . . . . . . . . . . . . . . . . . . 1

$P$ - and $S$-Wave Travel-Time Data . . . . . . . . . . . . . . . . . . . . . 4

Velocity Profiles . . . . . . . . . . . . . . . . . . . . . . . . . . . . . . 4

Summary Velocity Profiles . . . . . . . . . . . . . . . . . . . . . . . . 6

Acknowledgments . . . . . . . . . . . . . . . . . . . . . . . . . . . . . . . . . 13

References . . . . . . . . . . . . . . . . . . . . . 13

Appendix A-Detailed Results:

Cerritos College Gymnasium . . . . . . . . . . . . . . . . . . . . . 14

Cerritos College Physical Science Bldg. . . . . . . . . . . . . . . . . . 21

Cerritos College Police Bldg. . . . . . . . . . . . . . . . . . . . . . 28

Corps of Engineer Station . . . . . . . . . . . . . . . . . . . . . . . 35

Hoover School . . . . . . . . . . . . . . . . . . . . . . . . . . . . 42

Lincoln School . . . . . . . . . . . . . . . . . . . . . . . . . . . . 49

Lincoln School Whittier . . . . . . . . . . . . . . . . . . 56

Los Alisos Adult School . . . . . . . . . . . . . . . . . . . . . . . . 63

Olive Junior High School . . . . . . . . . . . . . . . . . . . . . . . 70

San Bernadino Fire Station . . . . . . . . . . . . . . . . . . . . . . 77

Santa Anita Golf Course . . . . . . . . . . . . . . . . . . . . . . . 84

South Western Academy … . . . . . . . . . . . . . . . . . . . . 91

St. Paul High School . . . . . . . . . . . . . . . . . . . . . . . . . 98

Appendix B-Poisson's Ratios:

Cerritos College Gymnasium . . . . . . . . . . . . . . . . . . . . 105

Cerritos College Physical Science Bldg. . . . . . . . . . . . . . . . . . 106

Cerritos College Police Bldg. . . . . . . . . . . . . . . . . . . . . . 107

Corps of Engineer Station . . . . . . . . . . . . . . . . . . . . . 108

Hoover School . . . . . . . . . . . . . . . . . . . . . . . . . . . 109

Lincoln School . . . . . . . . . . . . . . . . . . . . . . . . . . . . 110

Lincoln School Whittier . . . . . . . . . . . . . . . . . . . . 111

Los Alisos Adult School . . . . . . . . . . . . . . . . . . . . . . . . 112

Olive Junior High School . . . . . . . . . . . . . . . . . . . . . . . 113

San Bernadino Fire Station . . . . . . . . . . . . . . . . . . . . . . 114

Santa Anita Golf Course . . . . . . . . . . . . . . . . . . . . . . . 115

South Western Academy . . . . . . . . . . . . . . . . . . . . . . . 116

St. Paul High School . . . . . . . . . . . . . . . . . . . . . . . . . 117 


\section{BOREHOLE P- AND S-WAVE VELOCITY AT THIRTEEN SITES IN SOUTHERN CALIFORNIA}

by

James F. Gibbs, David M. Boore, John C. Tinsley, and Charles S. Mueller

\section{INTRODUCTION}

The U.S. Geological Survey (USGS), as part of a program to aquire seismic velocity data at locations of strong-ground motion in earthquakes (e.g. Gibbs, et al., 2000), has investigated thirteen additional sites in the Southern California region. Of the thirteen sites, twelve are in the vicinity of Whittier, California and one is located in San Bernardino, California.

Several deployments of temporary seismographs were made after the Whittier Narrows, California earthquake of 1 October 1987 (Mueller et al., 1988). A deployment, between 2 October and 9 November 1987, was the motivation for selection of six of the drill sites. Temporary portable seismographs at Hoover School (HOO), Lincoln School (LIN), Corps of Engineers Station (NAR), Olive Junior High School (OLV), Santa Anita Golf Course (SAG) and Southwestern Academy (SWA), recorded significant aftershock data. These portable sites with the exception of Santa Anita Golf Course were co-sited with strong-motion recorders.

Stations at HOO, Lincoln School Whittier (WLB), Saint Paul High School (STP), Alisos Adult School (EXC), Cerritos College Gymnasium (CGM), Cerritos College Physical Science Building (CPS), and Cerritos College Police Building (CPB) were part of an array of digital strong-motion stations deployed from "bedrock" in Whittier to near the deepest part of the Los Angeles basin in Norwalk. Although development and siting of this new array (patially installed at the time of this writing) was generally motivated by the Whittier Narrows earthquake, these new sites (with the exception of HOO) were not part of any Whittier Narrows aftershock deployments. A similar new digital strong-motion site was installed at the San Bernardino Fire Station during the same time frame.

Velocity data were obtained to depths of about 90 meters at two sites, 30 meters at seven sites, and 18 to 25 meters at four sites. Lithology data from the analysis of cuttings and samples, was obtained from the two 90-meter deep holes and from five of the shallower holes to supplement the velocity interpretation. The two 90 -meter boreholes (SB1, CPB) have been instrumented with borehole seismometers for continuous monitoring of earthquake activity (Rogers, et al., 1998). No drill samples or cuttings were obtained from the other six sites but driller's logs were scanned for major changes noted there. The velocity models at those sites were interpreted using only the measured data and major changes in the driller's log as noted above.

The sites are shown in Figure 1 and listed in Table 1, which gives references to information regarding the strong-motion data. Several hundred strong-motion records of the main-shock were written by this moderate size earthquake $\left(M_{L}=5.9\right)$ making it important from a scientific and engineering prospective (Brady et al., 1988, Shakal et al., 1988). 


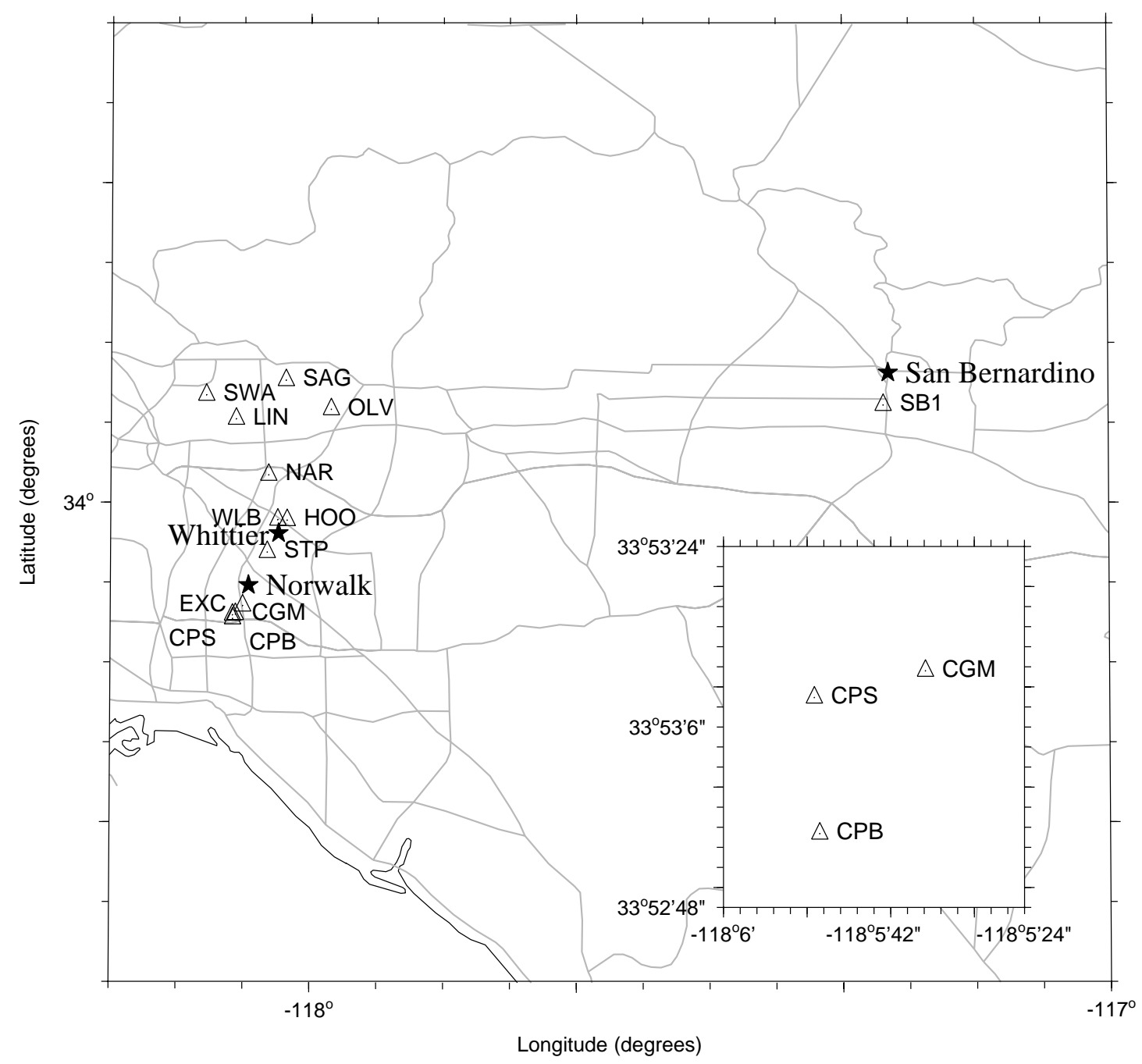

Figure 1. Regional map showing the locations of boreholes (triangles) included in this report. Inset shows the locations of the Cerritos College boreholes at an expanded scale. Locations of roads and cities are approximate. 
Table 1. Site names, three letter codes, and coordinates using the North American Datums of 1927 (NAD27) and 1983 (NAD83).

\begin{tabular}{llcccc}
\hline \multicolumn{1}{c}{ Station } & StaCode & Lat:NAD27 & Long:NAD27 & Lat:NAD83 & Long:NAD83 \\
\hline Cerritos College Gymnasium & CGM & 33.88663 & -118.09329 & 33.88664 & -118.09419 \\
Cerritos College Physical Sci. Bldg. & CPS & 33.88589 & -118.09698 & 33.88590 & -118.09788 \\
Cerritos College Police Bldg. & CPB & 33.88212 & -118.09680 & 33.88213 & -118.09770 \\
Corps of Engineers Station * & NAR & 34.03219 & -118.05225 & 34.03220 & -118.05315 \\
Hoover School * & HOO & 33.98491 & -118.02889 & 33.98492 & -118.02979 \\
Lincoln School * & LIN & 34.09043 & -118.09305 & 34.09044 & -118.09395 \\
Lincoln School Whittier & WLB & 33.98535 & -118.04061 & 33.98536 & -118.04151 \\
Los Alisos Adult School & EXC & 33.89559 & -118.08428 & 33.89560 & -118.08518 \\
Olive Junior High School * & OLV & 34.10073 & -117.97409 & 34.10074 & -117.97499 \\
San Bernardino Fire Station & SB1 & 34.10534 & -117.28201 & 34.10535 & -117.28289 \\
Santa Anita Golf Course & SAG & 34.13096 & -118.03074 & 34.13097 & -118.03164 \\
South Western Academy * & SWA & 34.11533 & -118.13046 & 34.11534 & -118.13136 \\
St. Paul High School & STP & 33.95158 & -118.05369 & 33.95159 & -118.05459 \\
\hline
\end{tabular}

* Strong-motion accelerograph located near borehole (see location maps in Appendix A). 


\section{$P$ - AND $S$-WAVE TRAVEL-TIME DATA}

Shear waves were generated at the ground surface by an air-powered horizontal ram (Liu, et al., 1988) striking an anvil at either end of an aluminum channel 2.3 meters long. The ram was driven first in one direction and then in the other to generate pulses of opposite polarity. A switch attached to the shear source triggered the recorder and established the reference for the timing of arrivals. $P$-waves were generated by striking a steel plate with a sledge hammer. The recorder was triggered by a switch attached to the handle of the sledge hammer. $P$ - and $S$-wave sources were offset from the borehole (same horizontal distance but different locations) to minimize the effect of waves traveling down the grout surrounding the casing. The source offsets varied from 2 to 4 meters depending on available space and depth of the borehole. Shallow holes (30 meters or less) were offset 2 or 3 meters.

Downhole measurements were made at 2.5-meter intervals at ten locations and at 2-meter spacing at three of the shallower boreholes. The measurements were made by moving a three-component geophone to each depth and clamping it to the casing by an electrically-activated lever arm. A second three-component geophone was placed on the surface near the shear source used to verify timing of the triggered recorder. The data were recorded on diskettes using a 12-channel recording system.

\section{VELOCITY PROFILES}

The procedure for determining velocities is summarized in Figure 2. Because the orientation of the downhole geophone could not be controlled when moving from one depth to the next, the azimuth of the horizontal geophones relative to the source was unknown and changed with depth. To minimize the effects of those changes, the horizontal components were rotated to the direction that maximized the integral square amplitude within a time interval containing the shear wave (Boatwright et al., 1986). $P$ - and $S$-wave first-arrival times were determined from the time series displayed at each depth on a 20inch computer screen. The $P$-wave arrival-time was obtained from the vertical trace, and the $S$-wave arrival-times were obtained from the average of the rotated horizontal traces for ram strikes in opposite directions. The arrivals were timed to the nearest millisecond, probably a realistic precision for clear arrivals uncontaminated by noise.

A trial set of layer boundaries was chosen for the S-wave model, based on the lithologic descriptions and geophysical logs at the two sites (CBP, SB1) where geologic information was available. At five sites (CGM, CPS, EXC, STP, WLB) simplified lithology, determined from drill cuttings, was used to supplement the velocity determinations. At the remaining six sites (NAR, HOO, LIN, OLV, SAG, SWA) the velocity models were determined without the benefit of lithology or electric logs. The travel-time data were fit in a least-squares sense by a model made up of constant velocity layers, taking into account refraction across the interfaces between layers. The travel times were weighted by the inverse of an assigned normalized variance. A normalized standard deviation of 1 was assigned to the clear arrivals and values up to 5 were assigned to the others. The residuals were examined, and layer boundaries were added, if necessary, to reduce large residuals or to remove systematic trends in the residuals. The $P$-wave travel time data were analyzed initially with the set of layer boundaries finally determined for the $S$-wave data. Layer boundaries were then added if needed to fit the data and deleted if not needed. Commonly, an additional layer 

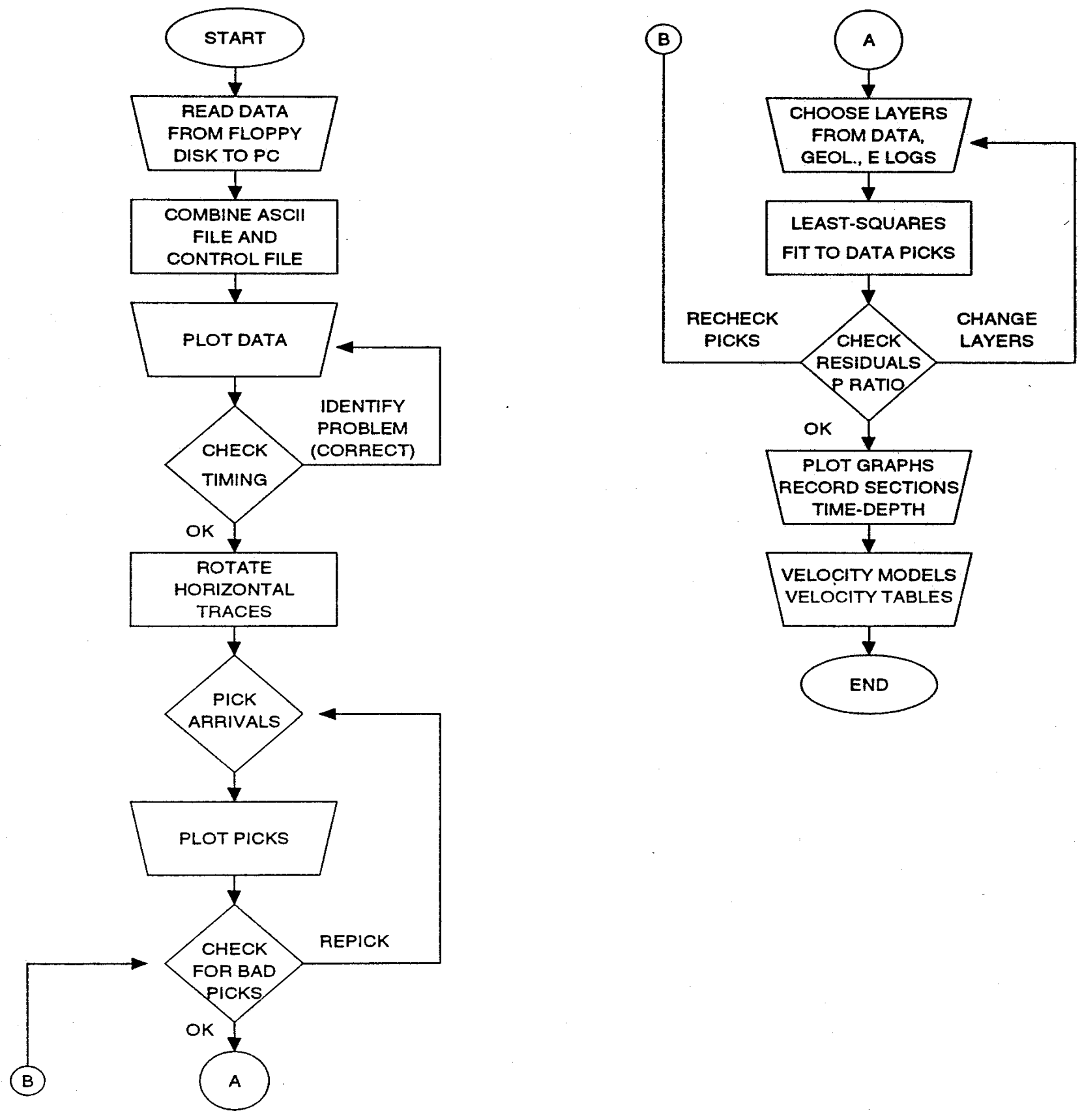

Figure 2. Flow-chart outlining the data processing and steps in the interpretation. 
boundary corresponding to the top of the zone of water saturation was needed to fit the $P$-wave data.

Some of the dynamic Poisson's ratios $\sigma$, calculated with initial velocity models, resulted in ratios that were out of the accepted range of values (0.0-0.5). To obtain a value in the acceptable range we made minor adjustments to the velocities using one or more of the following procedures: repicking shallow arrivals (usually $\mathrm{P}$ arrivals because small changes in $\mathrm{P}$ travel-times have greater effect on $\sigma$ ), adding a shallow layer, and/or adjusting layer thickness to ensure that Poisson's ratio was in the range 0.0-0.5. In most cases the small changes were made in the $\mathrm{P}$-wave velocities at shallow depths (for more details see, Gibbs, et al., 1999). Overall, the changes in velocity required to produce acceptable values of $\sigma$ were small and were only in a few layers.

For example, at San Bernardino Fire Station several velocity models were tried to get Poisson's ratio into the accepted range. We were forced to average the P-wave velocity over the top 8.5 meters to get the ratio from a negative value to a value of 0.04 . The preferred model in which the S-velocity follows the lithology (in general, the S-wave velocity is a better indicator of lithology than P-wave velocity) is included in Appendix A.

\section{SUMMARY VELOCITY PROFILES}

Figures 3-5 show the $S$-wave velocity profiles determined from the borehole measurements at the thirteen sites. The velocity profiles are plotted at the same scale for ease of comparison. Figures $6-8$ show the $P$-wave velocity profiles for the same sites as Figures $3-5$, respectively.

\section{DESCRIPTION OF APPENDICES}

Appendix A contains for each site: a location map, $S$ - and $P$-wave time-series records, a time-depth plot, and tables giving arrival times and velocity values. The upper and lower bounds on the velocity plots show approximate 68 percent confidence limits. The bounds are not symmetrical because they are based on the inverse velocities in the layers. Appendix B contains tables of P- and S-wave velocity models and the Poisson's ratios obtained from those models. 


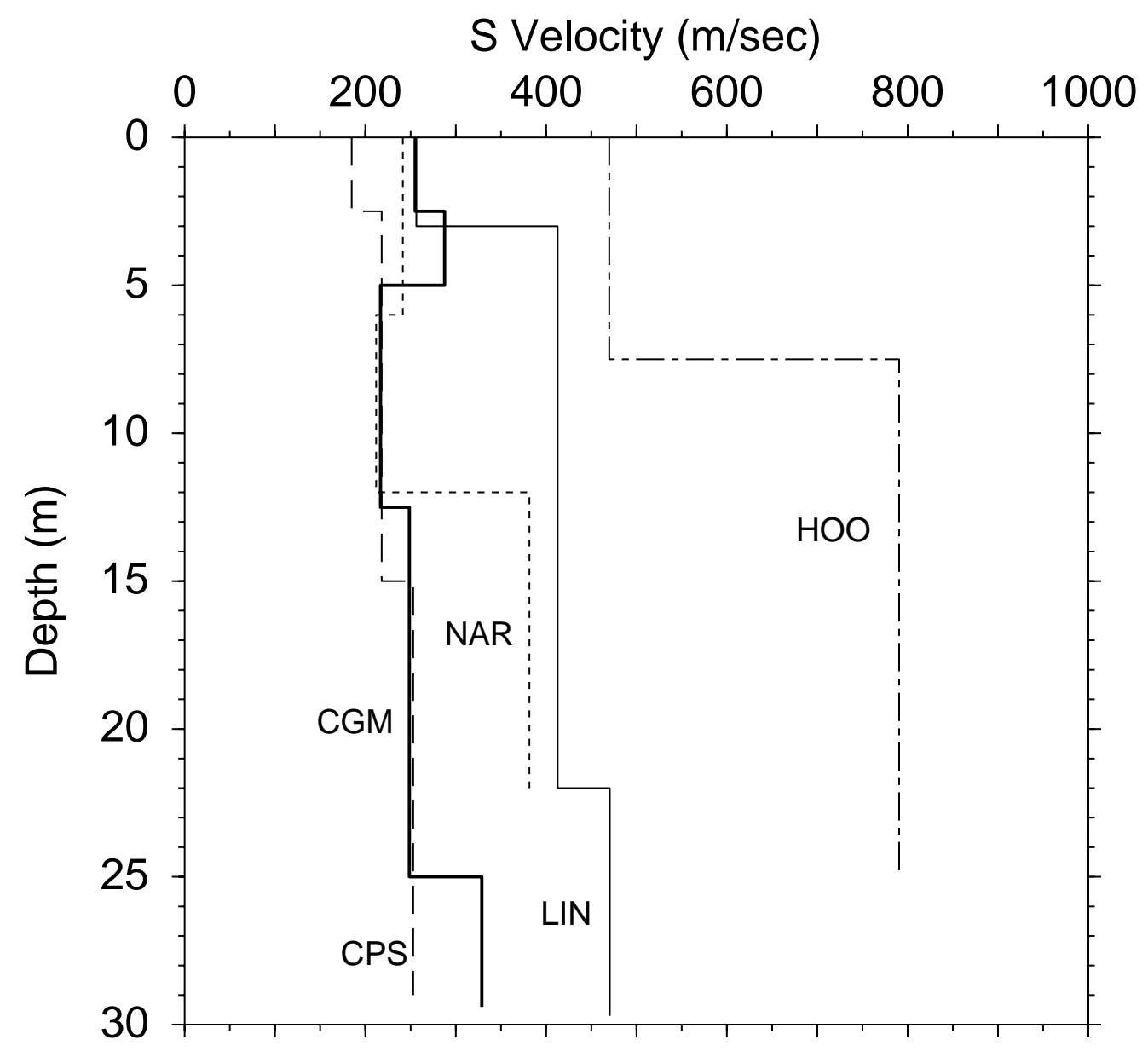

Figure 3. S-wave velocity models shown on the same figure for comparison. 


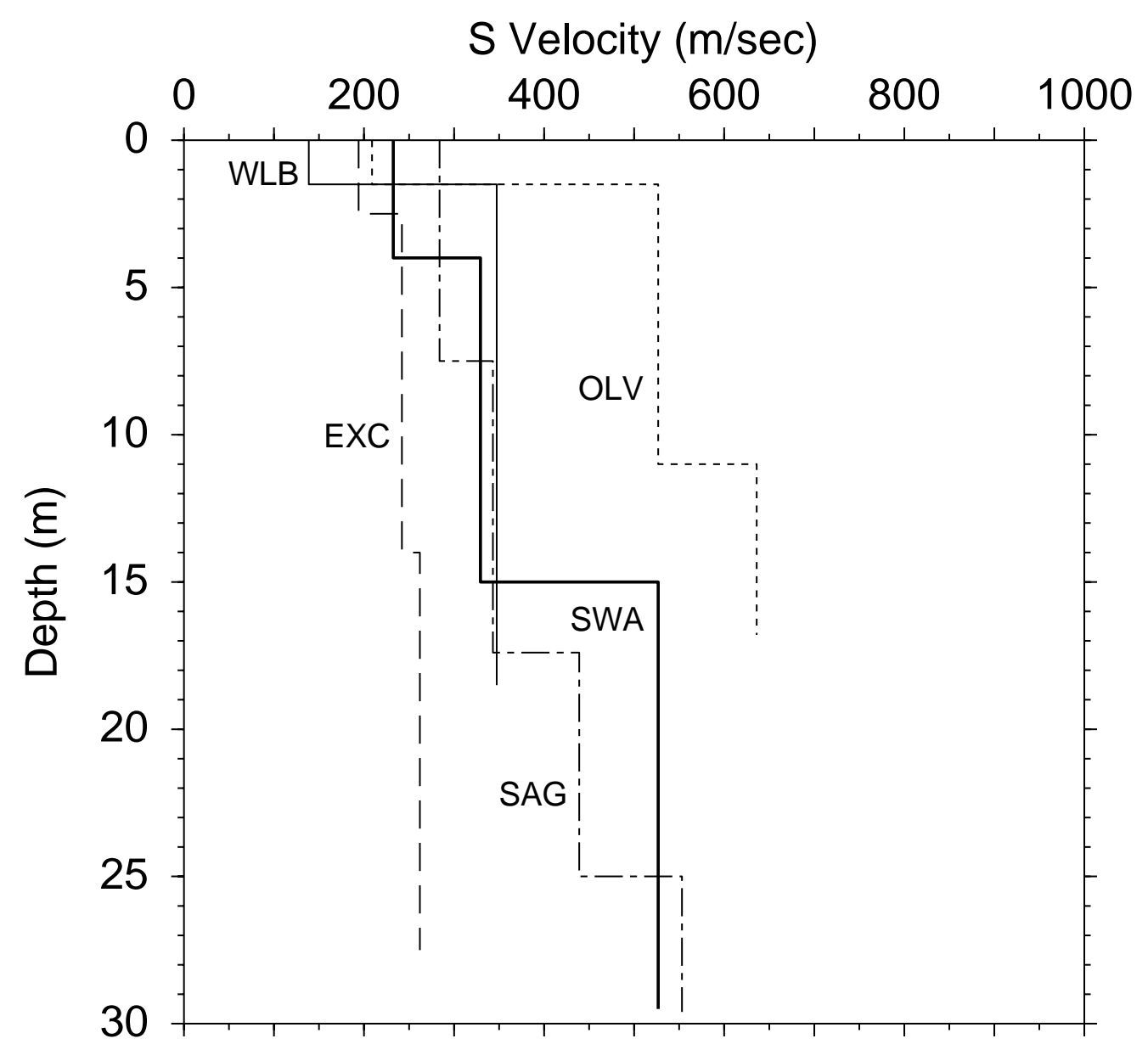

Figure 4. S-wave velocity models shown on the same figure for comparison. 


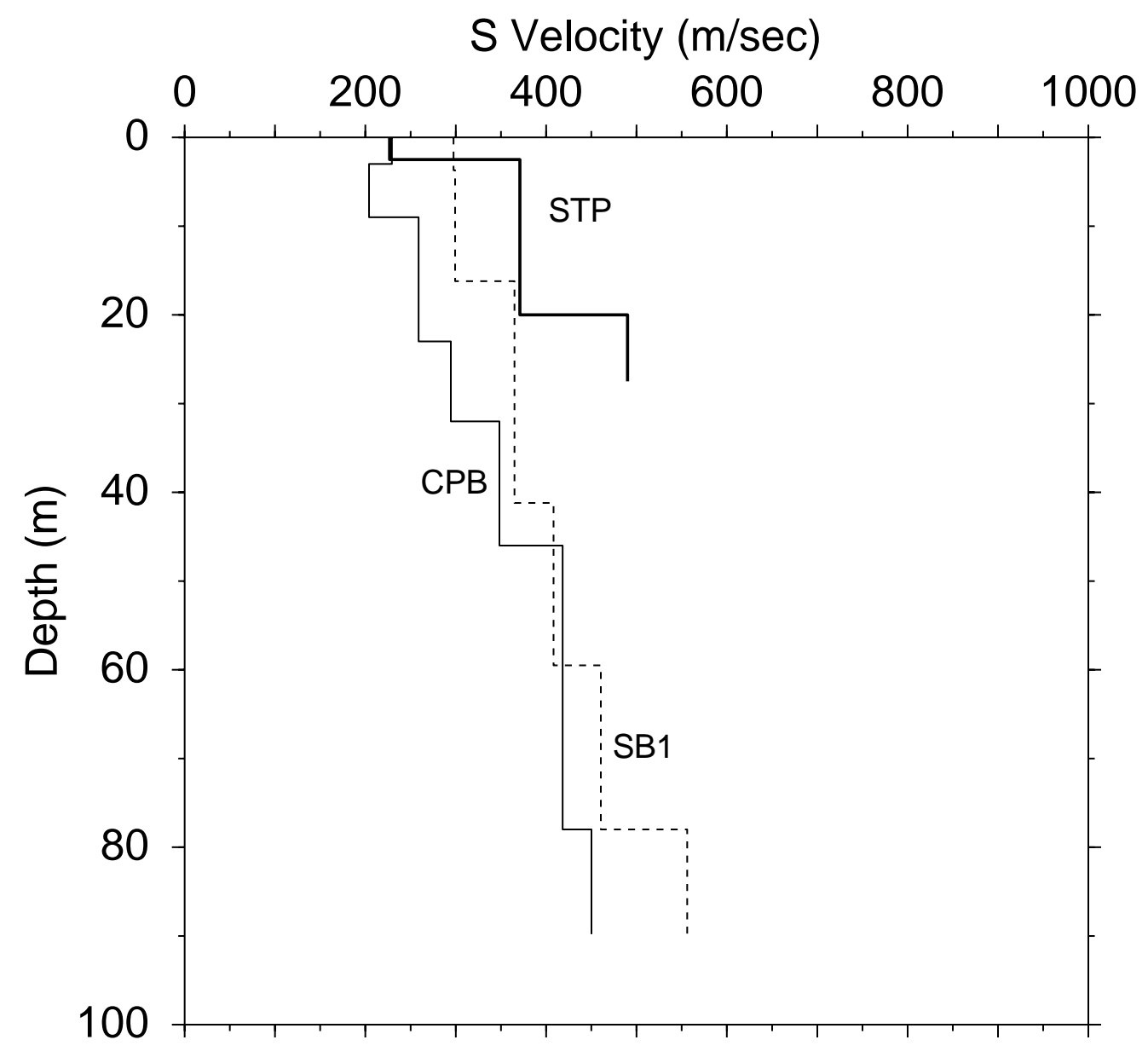

Figure 5. S-wave velocity models shown on same figure for comparison. 


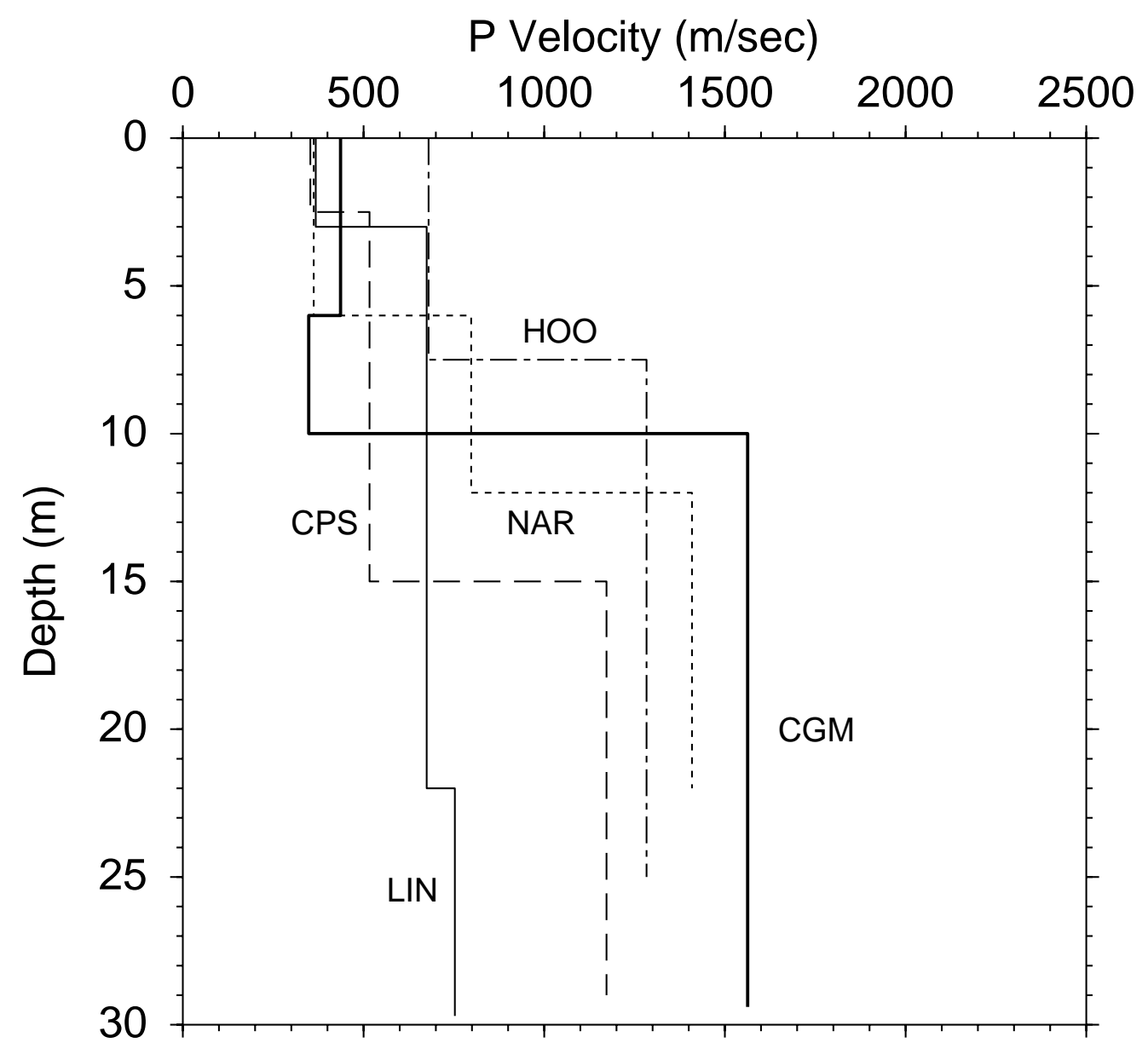

Figure 6. P-wave velocity models shown on the same figure for comparison. 


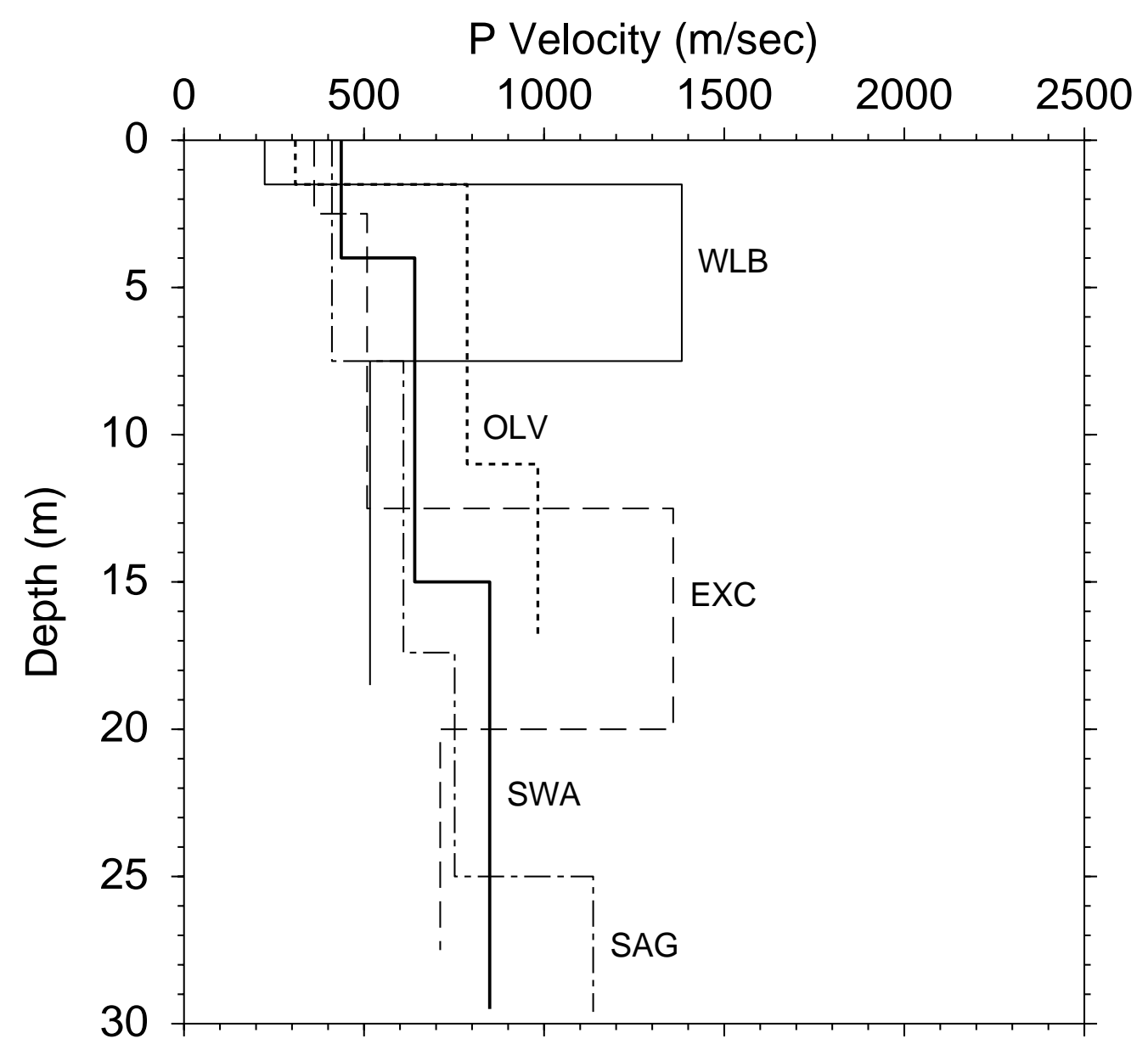

Figure 7. P-wave velocity models shown on the same figure for comparison. 


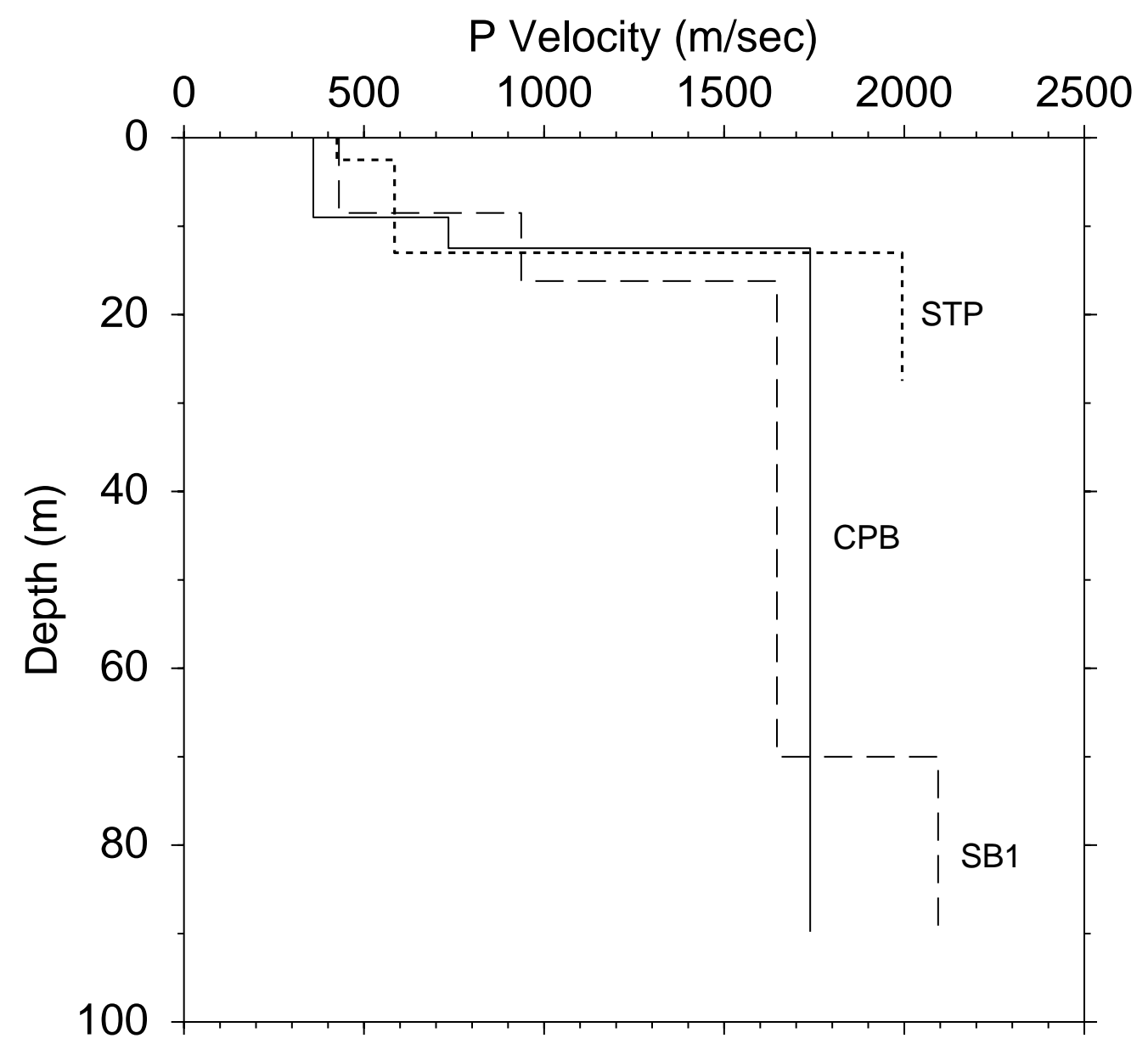

Figure 8. P-wave velocity models shown on the same figure for comparison. 


\section{ACKNOWLEDGMENTS}

We could not have completed these studies without the assistance of many individuals who helped us to gain access to the sites, assisted with utilities clearances and granted permission to conduct the studies. These people include Michael Sebak at Cerritos College; Warren Thomas at Corps of Engineers Station; Margie Leon and Ray Rodriguez at Hoover School; Jack Feldman at Lincoln School; Stephen Finkle at Lincoln School Whittier; Mr. Hengler at Los Alisos Adult School; Daniel at Olive Junior High School; Richard McGreevy at San Bernardino Fire Station; Dave Cuellar, Terry Moeller, and Tom Dittmar at Santa Anita Golf Course; Charles Craig at South Western Academy; Father Robert Gallagher at St. Paul High School. We also thank Allen Foss of the U.S. Geological Survey for his help with the P- and S-wave logging.

\section{REFERENCES}

Boatwright, John, Porcella, R., Fumal, T., Liu, Hsi-Ping, (1986), Direct estimates of shear wave amplification from a borehole near Coalinga, California: Earthquake Notes, v. 57, p. 8.

Brady, A. G., Etheredge, E. C., and Porcella, R. L. (1988), The Whittier Narrows, California Earthquake of October 1, 1987, Prelininary assessment of strong ground motion records: Earthquake Spectra, v.4, no.1, p. 55-74.

Gibbs, James F., Tinsley, John C., Boore, David M., and Joyner, William B., 1999, Seismic velocities and geological conditions at twelve sites subjected to strong ground motion in the 1994 Northridge, California, earthquake: a revision of OFR 96-740, U.S. Geological Survey, Open-File Report 99-446, 142p.

Gibbs, James F., Tinsley, John C., Boore, David M., and Joyner, William B., 2000, Borehole velocity measuremnets and geological conditions at thirteen sites in the Los Angeles, California region: U.S. Geological Survey, Open-File Report OF 00470, 118p.

Liu, Hsi-Ping, Warrick, Richard E., Westerlund, Robert E., Fletcher, Jon B. and Maxwell, Gary L., 1988, An air-powered impulsive shear-wave source with repeatable signals: Bull. Seism. Soc. Am. v. 78, p. 355-369.

Mueller, C., Dietel, C., Glassmoyer, G., Noce, T., Sembera, E., Spudich, P., and Watson, J., Digital Recordings of Aftershocks of the 1 October 1987 Whittier Narrows, California, Earthquake: U.S. Geological Survey, Open-File Report 88-688, 40p.

Rogers, A., Mueller, C., Tinsley, J., and Koesterer, C., (1998), Instrumentation and geotechnical characteristics at two vertical strong motion arrays at Cerritos College, Norwalk, and downtown San Bernardino, California: U.S. Geological Survey, OpenFile Report 98-346, 58p.

Shakal, A., Huang, M. J., and Cao, T. Q. (1988), The Whittier Narrows, California Earthquake of October 1, 1987, CSMIP strong motion data: Earthquake spectra, v.4, no.1, p. 75-100. 
APPENDIX - A

Detailed Results 


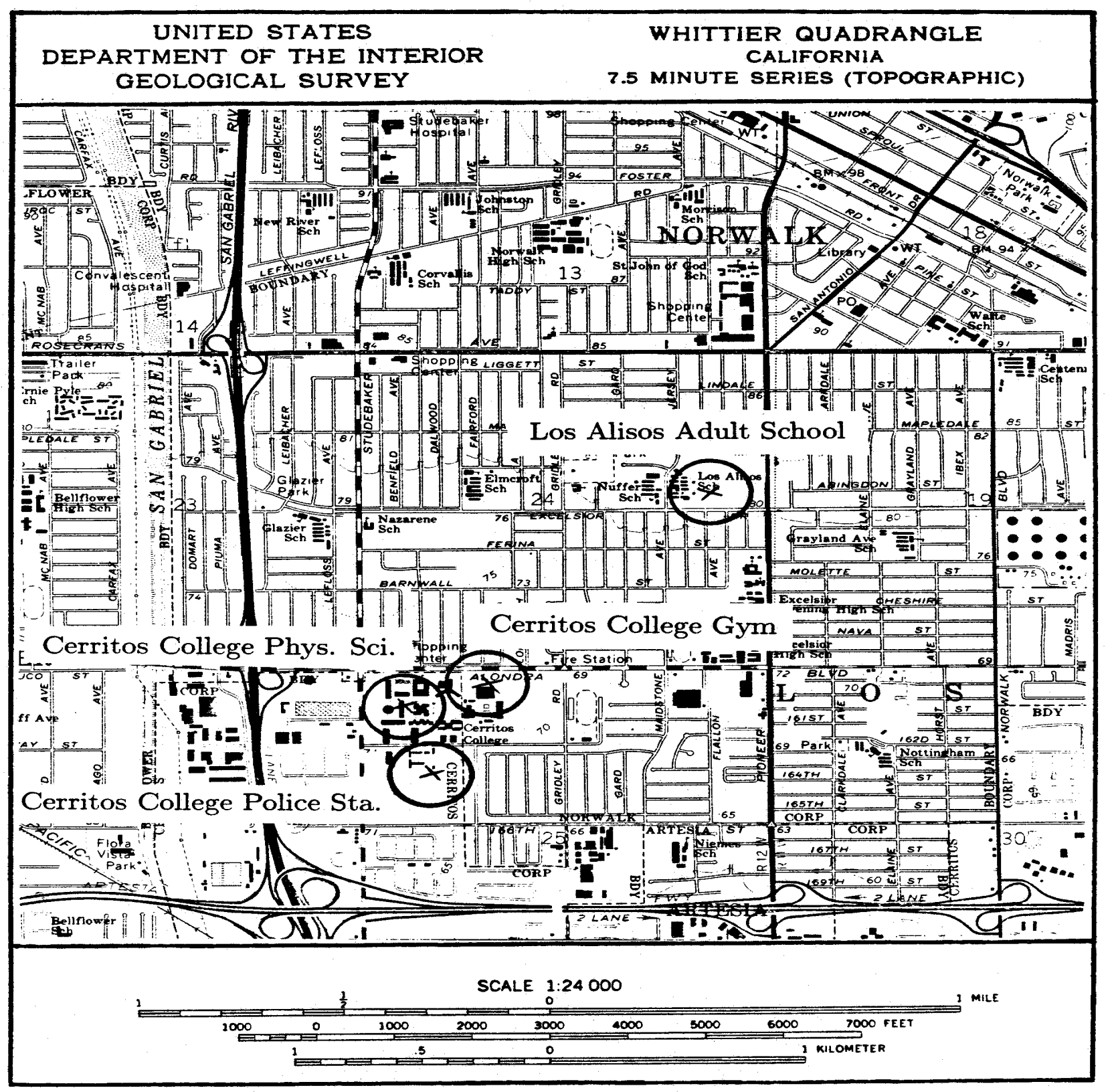

Figure A-1. Site location map for the borehole at Cerritos College Gymnasium. 


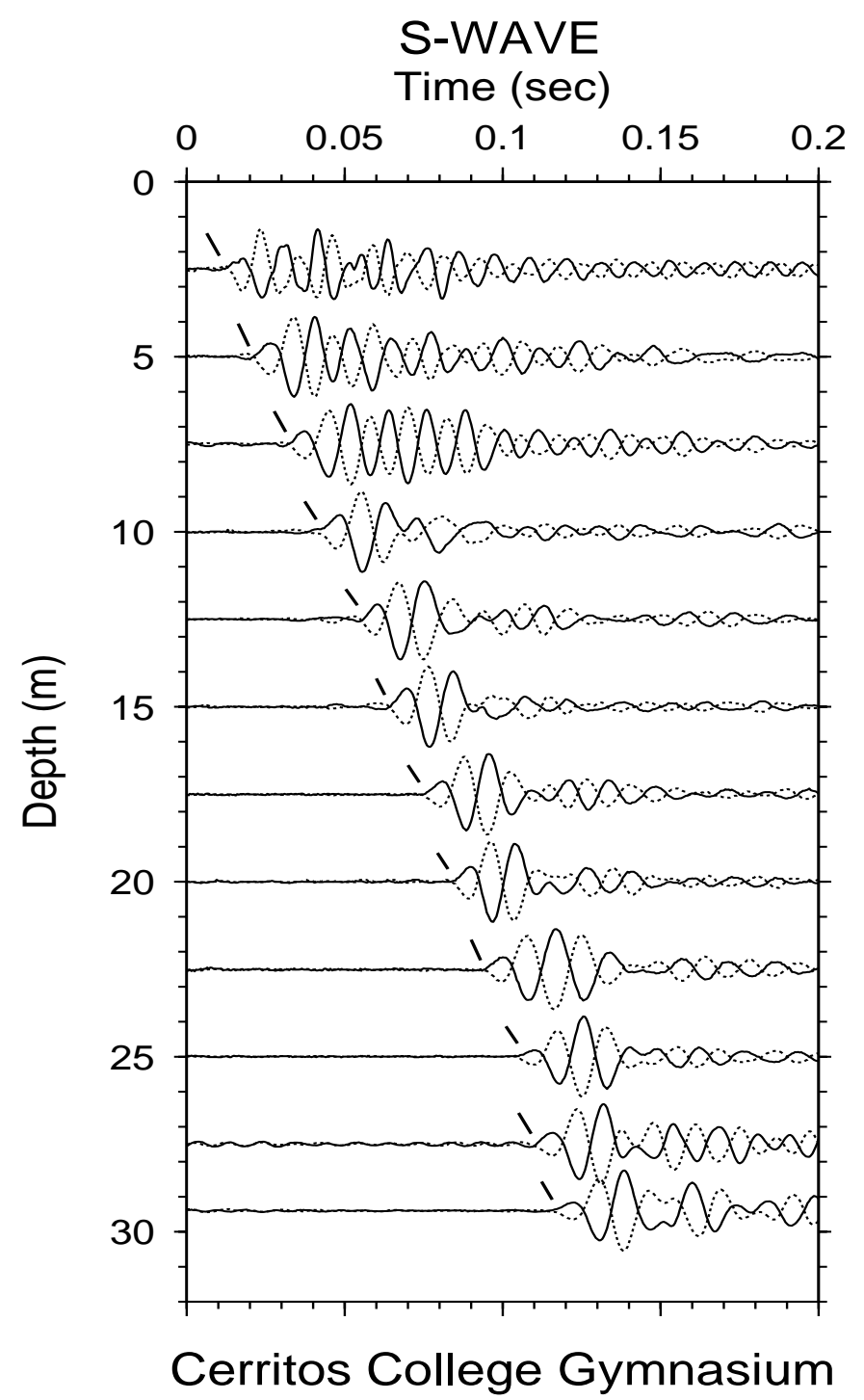

Oct 23, 2001

Figure A-2. Horizontal component record section (from impacts in opposite directions) superimposed for identification of S-wave onset. Approximate S-wave time picks are indicated by the hatch marks. 


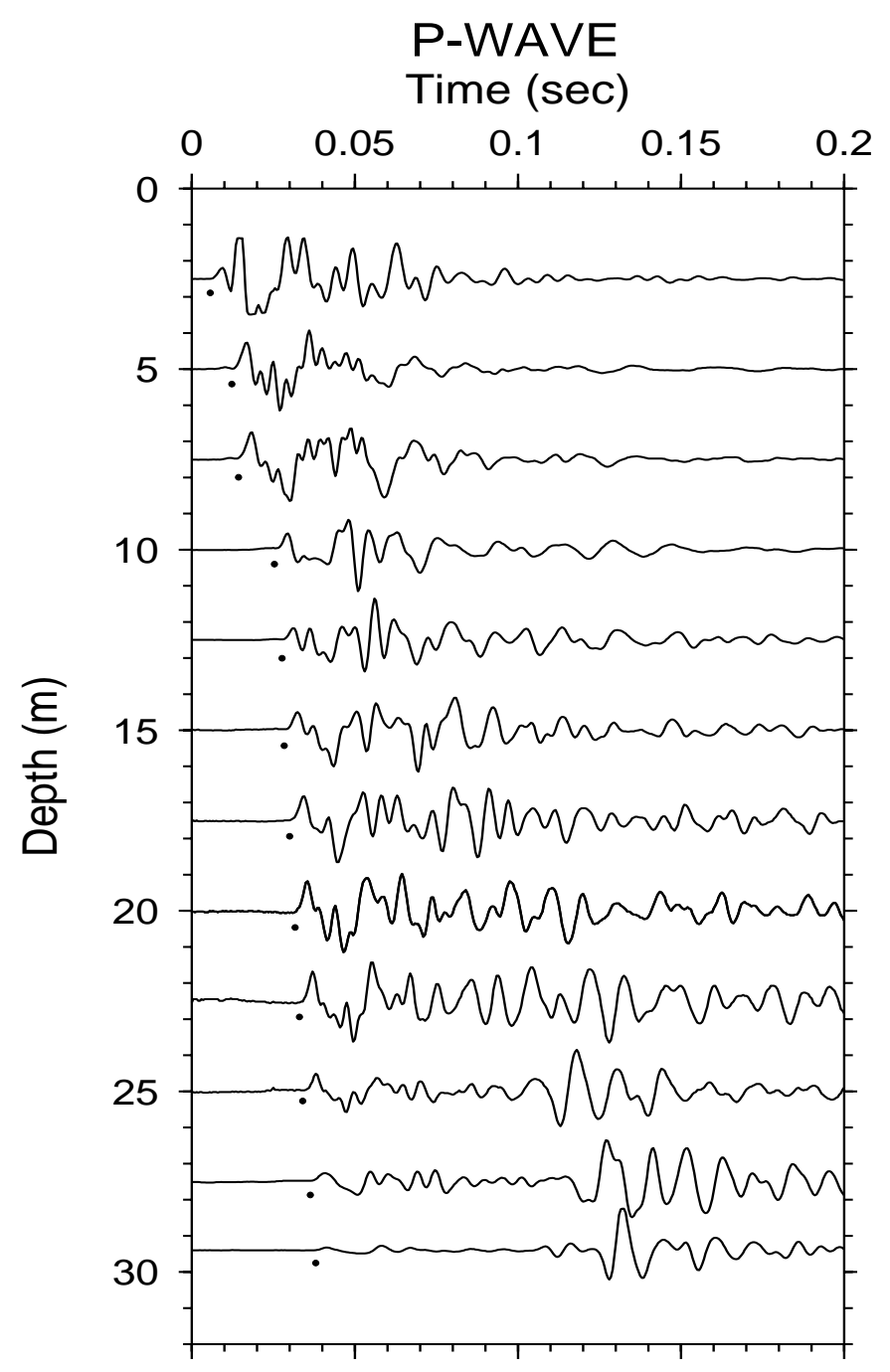

Cerritos College Gymnasium

Oct 23, 2001

F:ICGMICGMPWAVE.DT F:ICGMICGMPWAVE.GRA

Figure A-3. Vertical component record section. Approximate P-wave arrivals are indicated by the dots. 


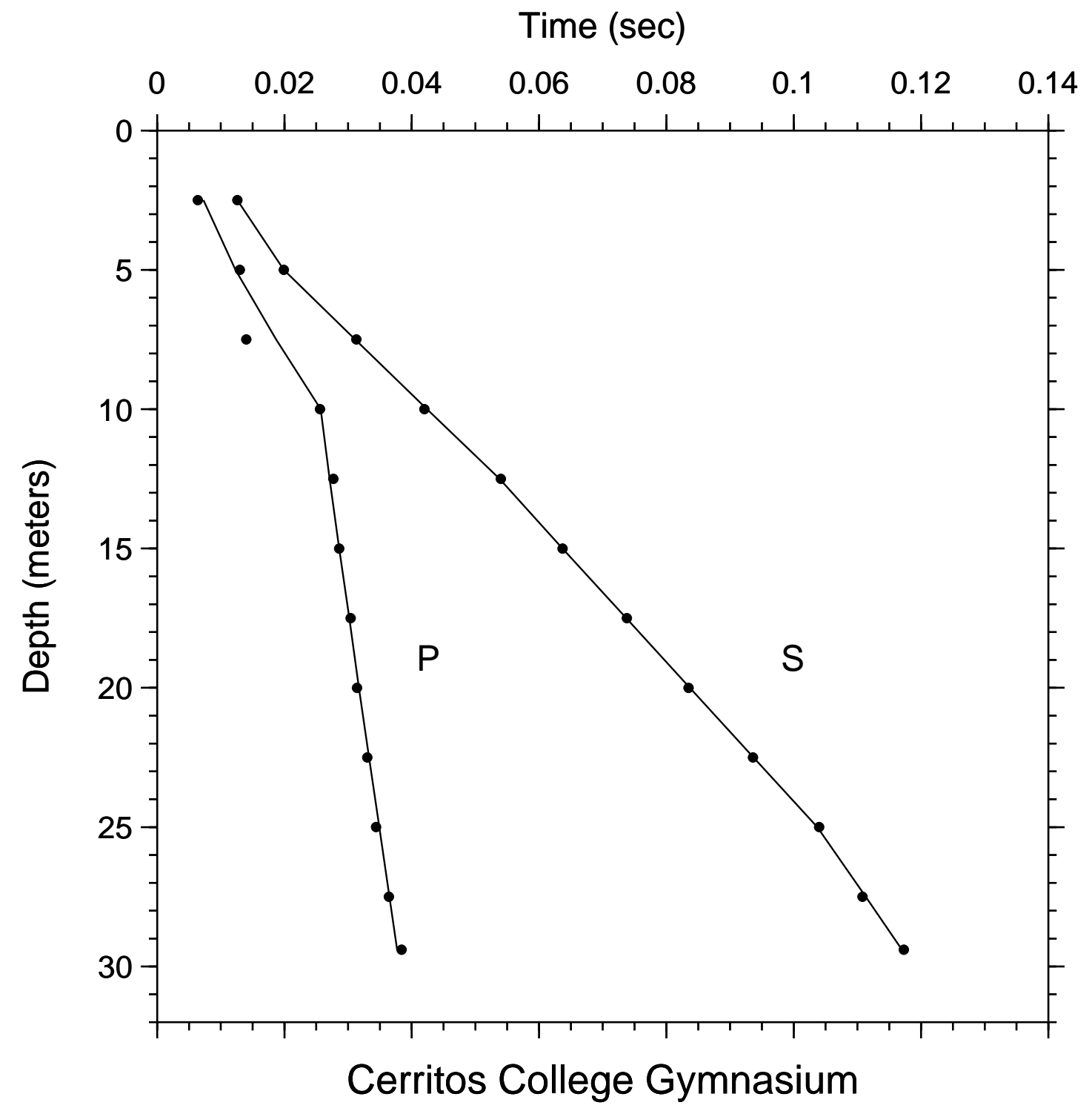

Figure A-4. Time-depth graph of P-wave and S-wave picks. Line segments are straightline interpolations of model predictions at the observation depths. The times for zero depth, not shown, are given by hoffset divided by the velocity in the uppermost layer (see accompanying tables of velocities for specific values). 


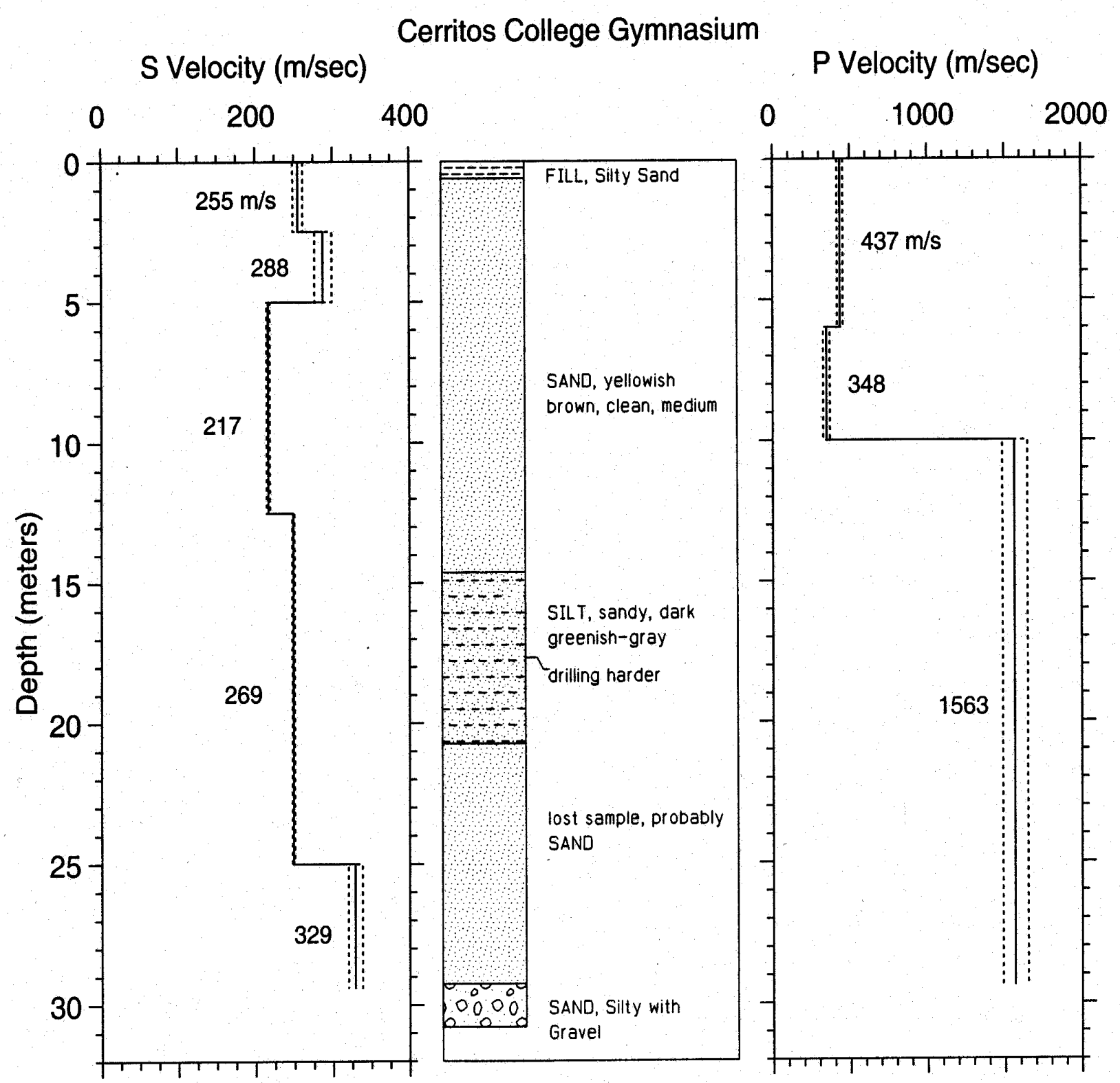

Figure A-5. S- and P-wave velocity profiles with dashed lines representing plus and minus one standard deviation. Generalized geologic log is shown for correlation with velocities. 
ABLE A-1. S-wave arrival times and velocity sumaries.

Location: Cerritos College Gyunasium: S Coordinates: hoffset $=2.00$ travel-time file: $\mathrm{P}: \backslash \mathrm{CGM} \backslash \mathrm{CGMS} . \mathrm{TT}$

$\begin{array}{rrrrcrr}d(\mathrm{I}) & \mathrm{d}(\mathrm{ft}) & \mathrm{tsl}(s) & \mathrm{twrt}(s) & \text { vavg }(\mathrm{m} / \mathrm{s}) & \text { sig } & \mathrm{rsdl}(\mathrm{sec}) \\ 2.5 & 8.2 & 0.0126 & 0.0098 & 255 & 1 & 0.0000 \\ 5.0 & 16.4 & 0.0199 & 0.0185 & 270 & 1 & 0.0000 \\ 7.5 & 24.6 & 0.0313 & 0.0300 & 250 & 1 & 0.0002 \\ 10.0 & 32.8 & 0.0420 & 0.0415 & 241 & 1 & -0.0004 \\ 12.5 & 41.0 & 0.0540 & 0.0530 & 236 & 1 & 0.0002 \\ 15.0 & 49.2 & 0.0637 & 0.0631 & 238 & 1 & 0.0000 \\ 17.5 & 57.4 & 0.0738 & 0.0731 & 239 & 1 & 0.0001 \\ 20.0 & 65.6 & 0.0835 & 0.0832 & 240 & 1 & -0.0002 \\ 22.5 & 73.8 & 0.0936 & 0.0932 & 241 & 1 & -0.0001 \\ 25.0 & 82.0 & 0.1040 & 0.1032 & 242 & 1 & 0.0003 \\ 27.5 & 90.2 & 0.1108 & 0.1108 & 248 & 1 & -0.0005 \\ 29.4 & 96.5 & 0.1173 & 0.1166 & 252 & 1 & 0.0003\end{array}$

$33.88663-118.09329$ Hole_Code: 296 nlayers $=5$

dtb (II) thk(II) $\mathrm{v}(\mathrm{II} / \mathrm{s}) \mathrm{vl}(\mathrm{II} / \mathrm{s}) \quad \mathrm{vu}(\mathrm{II} / \mathrm{s})$

$\begin{array}{lllll}2.5 & 2.5 & 255 & 249 & 262\end{array}$

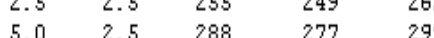

$\begin{array}{rrrrr}12.5 & 7.5 & 217 & 217 & 299 \\ 219 & 219\end{array}$

$25.0 \quad 12.5 \quad 249 \quad 247 \quad 250$

$\begin{array}{lrrrr}29.4 & 4.4 & 329 & 320 & 338\end{array}$

dtb (ft) thk (ft) $\mathrm{v}(\mathrm{ft} / \mathrm{s}) \quad \mathrm{vl}(\mathrm{ft} / \mathrm{s}) \quad \mathrm{vu}(\mathrm{ft} / \mathrm{s})$ $\begin{array}{lllll}8.2 & 8.2 & 837 & 817 & 858\end{array}$ $\begin{array}{lrrrr}16.4 & 8.2 & 943 & 908 & 981\end{array}$ $1.0-24.6-711$ $\begin{array}{rrrrr}82.0 & 41.0 & 816 & 811 & 822 \\ 96.5 & 14.4 & 1079 & 1050 & 1109\end{array}$

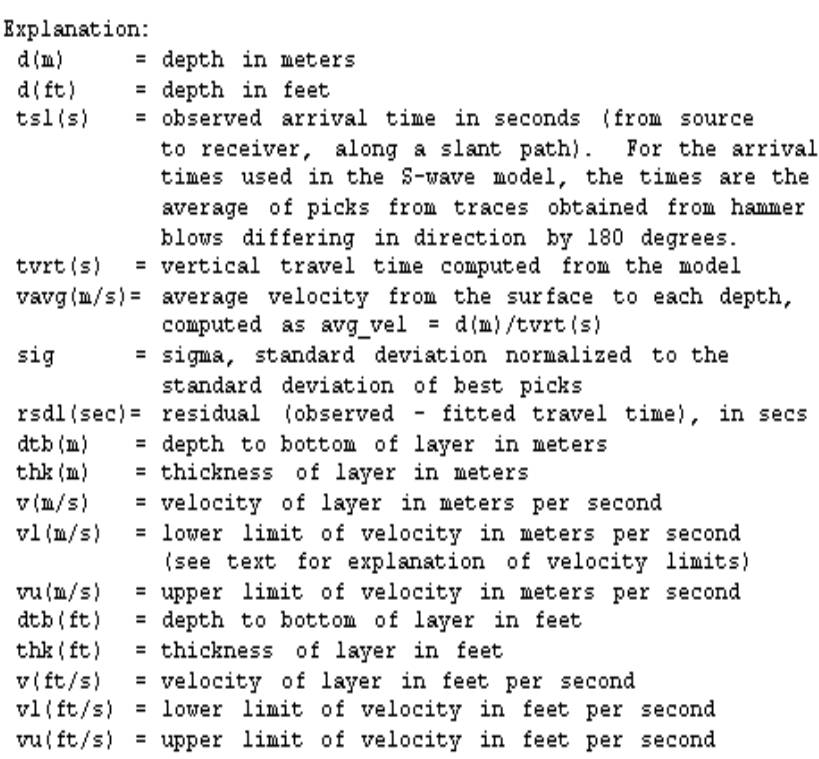


TABELE A-2. P-wave arrival times and velocity summaries.

Location: Cerritos College Gyunasium: p Coordinates: hoffset $=2.00$ travel-time file: $\mathrm{F}: \backslash$ CGM $\backslash$ CGMP.TT

$\begin{array}{rrrrcrr}d(\mathbb{I}) & d(\mathrm{ft}) & t s 1(s) & t v r t(s) & \text { vavg }(\mathbb{m} / s) & s i g & \mathrm{rsdl}(\mathrm{sec}) \\ 2.5 & 8.2 & 0.0064 & 0.0057 & 437 & 1 & -0.0009 \\ 5.0 & 16.4 & 0.0130 & 0.0114 & 437 & 1 & 0.0007 \\ 7.5 & 24.6 & 0.0140 & 0.0180 & 416 & 5 & -0.0047 \\ 10.0 & 32.8 & 0.0256 & 0.0252 & 396 & 1 & -0.0001 \\ 12.5 & 41.0 & 0.0277 & 0.0268 & 466 & 1 & 0.0006 \\ 15.0 & 49.2 & 0.0286 & 0.0284 & 528 & 1 & -0.0001 \\ 17.5 & 57.4 & 0.0304 & 0.0300 & 583 & 1 & 0.0002 \\ 20.0 & 65.6 & 0.0314 & 0.0316 & 632 & 1 & -0.0004 \\ 22.5 & 73.8 & 0.0330 & 0.0332 & 677 & 1 & -0.0003 \\ 25.0 & 82.0 & 0.0344 & 0.0348 & 718 & 1 & -0.0005 \\ 27.5 & 90.2 & 0.0364 & 0.0364 & 755 & 1 & -0.0001 \\ 29.4 & 96.5 & 0.0384 & 0.0376 & 781 & 1 & 0.0007\end{array}$

$33.88663-118.09329$ Hole_Code: 296 nlayers $=3$

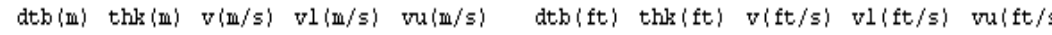
$\begin{array}{rlllllllll}6.0 & 6.0 & 437 & 418 & 456 & 19.7 & 19.7 & 1432 & 1372 & 1497 \\ 10.0 & 4.0 & 348 & 328 & 371 & 32.8 & 13.1 & 1142 & 1075 & 1219\end{array}$

$\begin{array}{rrrrrrrrrr}10.0 & 4.0 & 348 & 328 & 371 & 32.8 & 13.1 & 1142 & 1075 & 1219 \\ 29.4 & 19.4 & 1563 & 1486 & 1648 & 96.5 & 63.6 & 5128 & 4877 & 5407\end{array}$




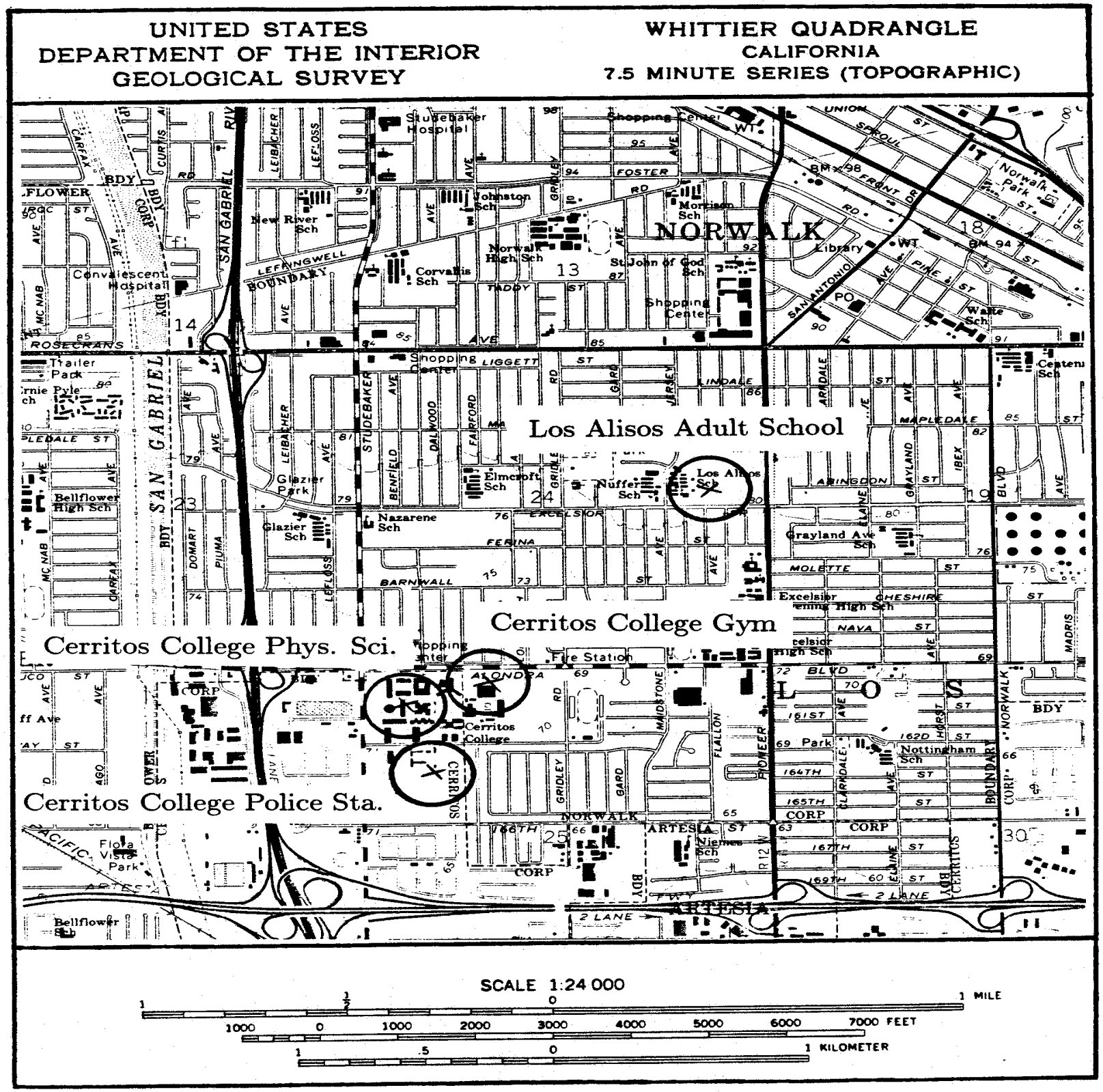

Figure A-6. Site location map for the borehole at Cerritos College Physical Science Building. 


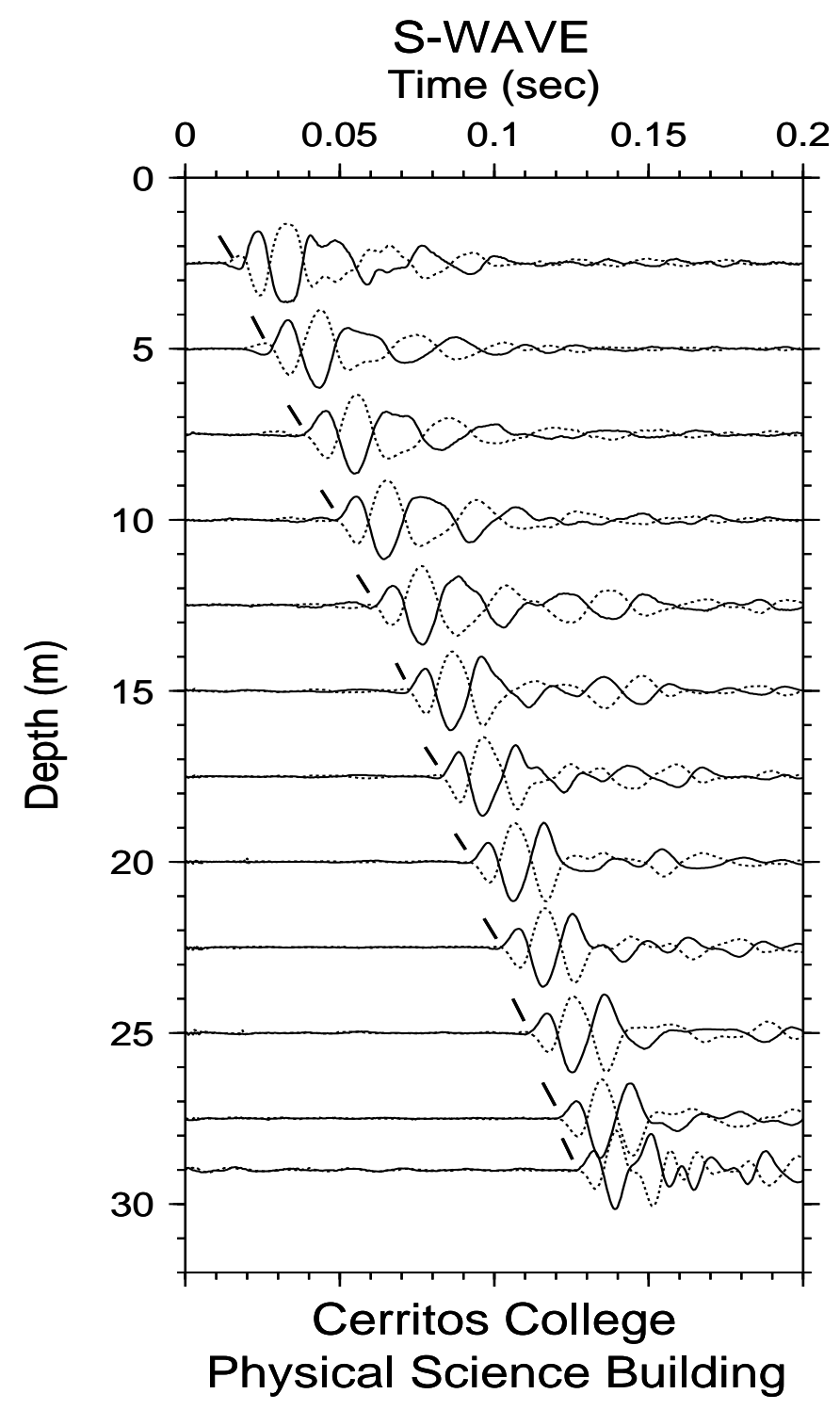

Figure A-7. Horizontal component record section (from impacts in opposite directions) superimposed for identification of S-wave onset. Approximate S-wave time picks are indicated by the hatch marks. 


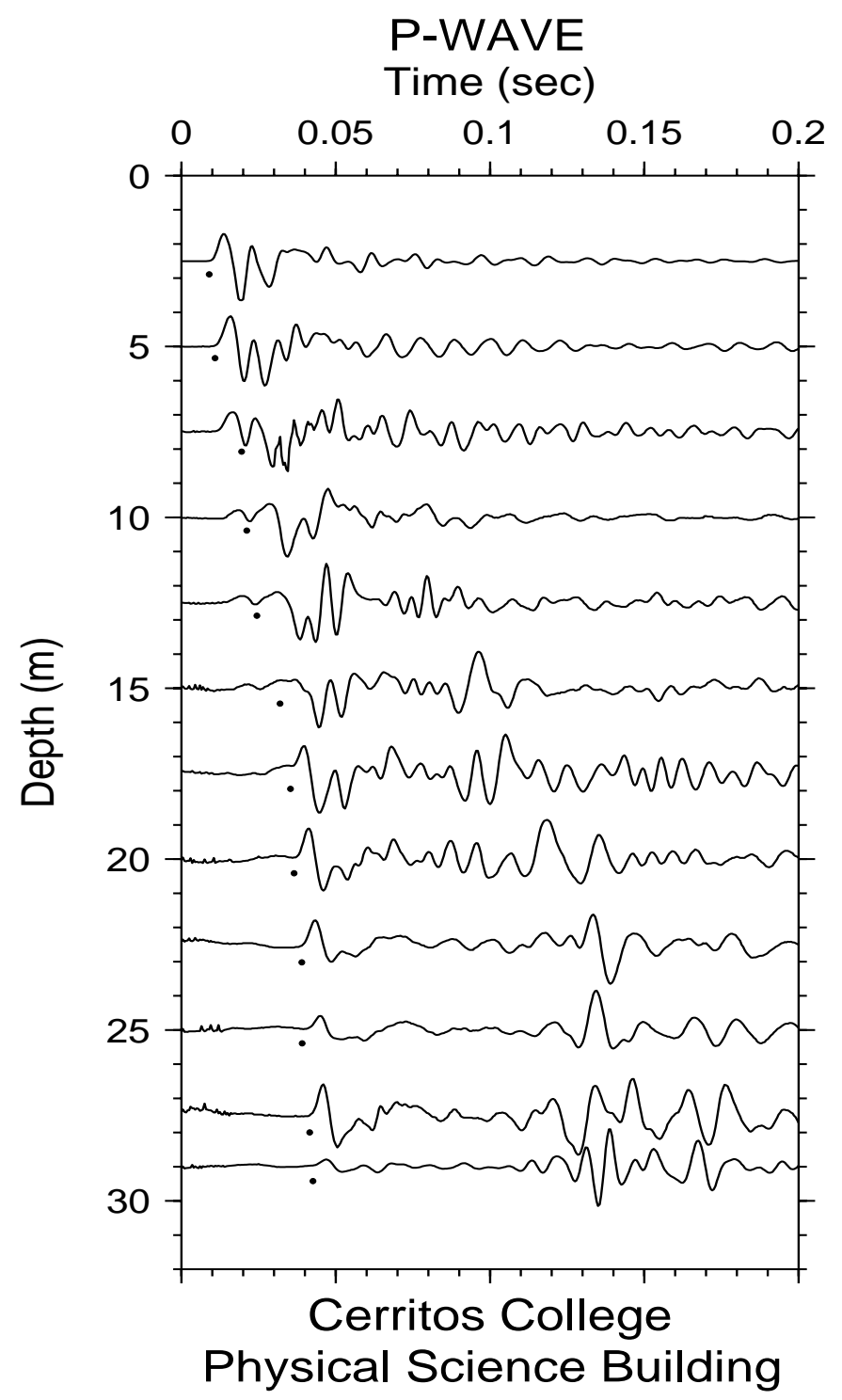

Figure A-8. Vertical component record section. Approximate $\mathrm{P}$-wave arrivals are indicated by the dots. 


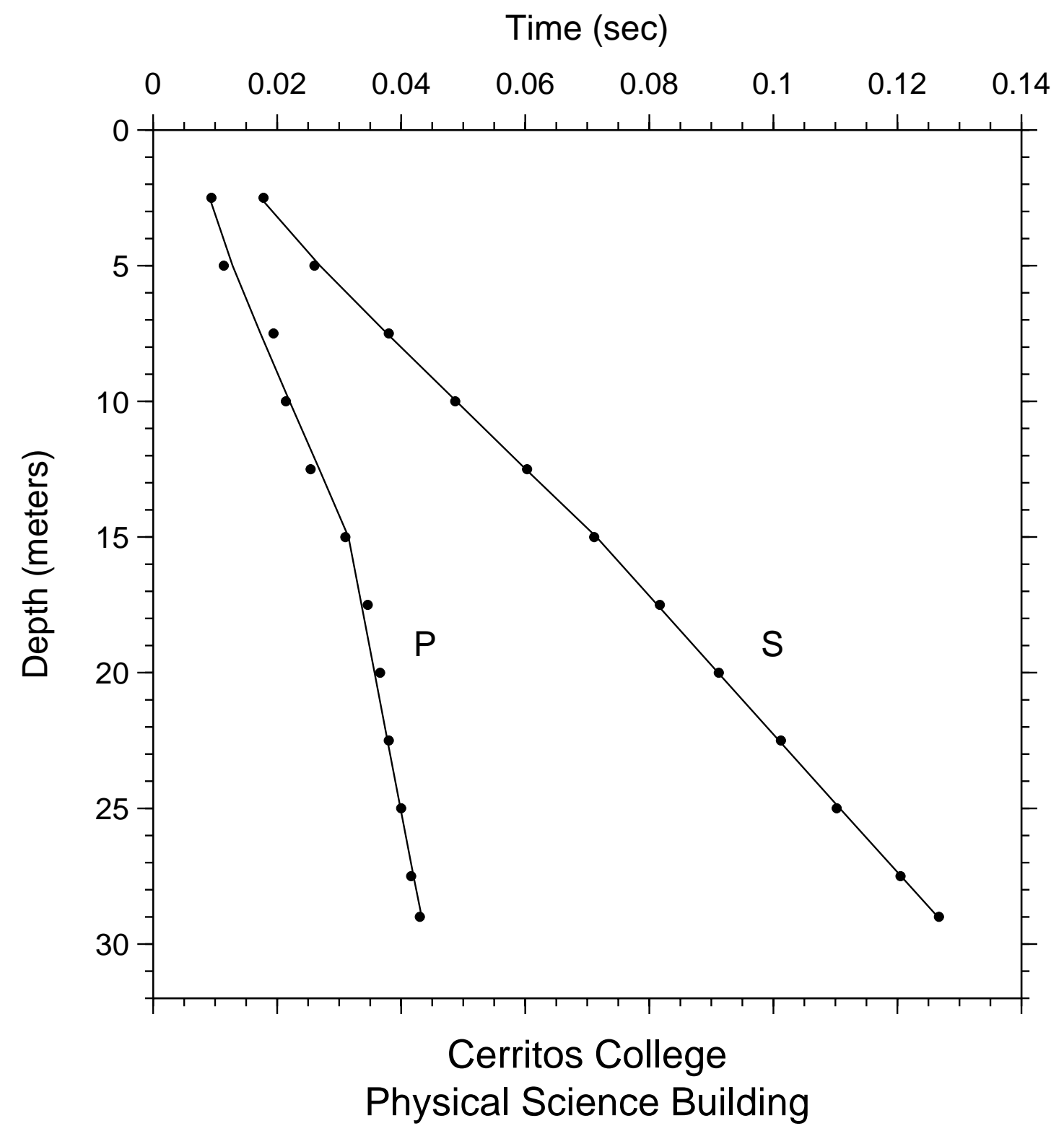

Figure A-9. Time-depth graph of P-wave and S-wave picks. Line segments are straightline interpolations of model predictions at the observation depths. The times for zero depth, not shown, are given by hoffset divided by the velocity in the uppermost layer (see accompanying tables of velocities for specific values). 


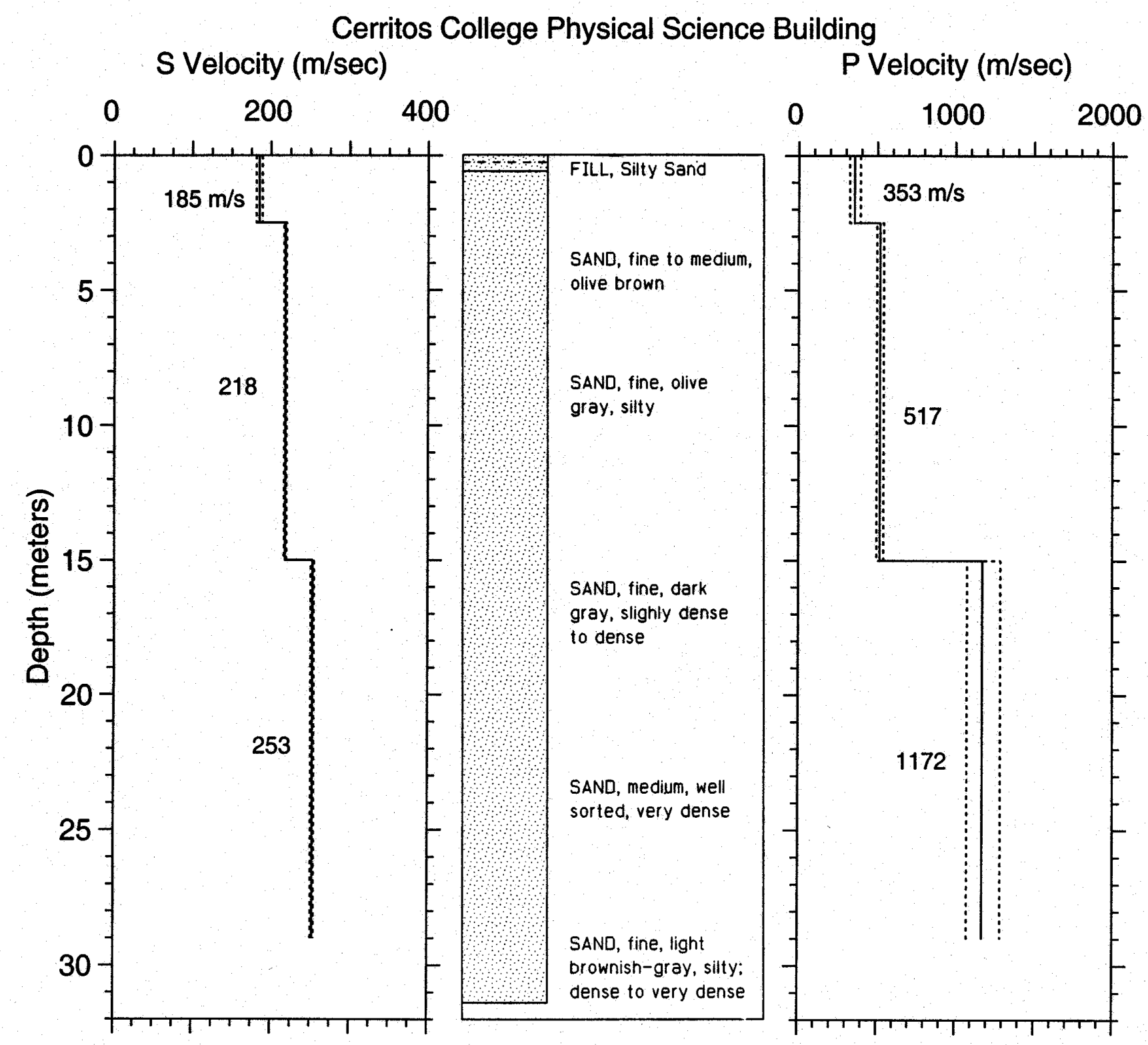

Figure A-10. S- and P-wave velocity profiles with generalized geologic log. Dashed lines represent plus and minus one standard deviation. 
ABLE A-3. S-wave arrival times and velocity sumaries.

Location: Physical Sciences Building: \& Coordinates: hoffset $=2.00$ travel-time file: $\mathrm{P}: \backslash$ CPS $\backslash$ CPSS.TT

$33.88589-118.09700$ Hole_Code: 297 nlayers $=3$

$\begin{array}{rrrrrrr}\mathrm{d}(\mathrm{m}) & \mathrm{d}(\mathrm{ft}) & \mathrm{tsl}(s) & \text { tvrt }(s) & \text { vavg }(\mathrm{m} / \mathrm{s}) & \text { sig } & \mathrm{rsdl}(\mathrm{sec}) \\ 2.5 & 8.2 & 0.0178 & 0.0135 & 185 & 1 & 0.0005 \\ 5.0 & 16.4 & 0.0260 & 0.0250 & 200 & 1 & -0.0009 \\ 7.5 & 24.6 & 0.0380 & 0.0364 & 206 & 1 & 0.0003 \\ 10.0 & 32.8 & 0.0487 & 0.0479 & 209 & 1 & -0.0002 \\ 12.5 & 41.0 & 0.0603 & 0.0594 & 210 & 1 & 0.0002 \\ 15.0 & 49.2 & 0.0711 & 0.0709 & 212 & 1 & -0.0004 \\ 17.5 & 57.4 & 0.0817 & 0.0807 & 217 & 1 & 0.0004 \\ 20.0 & 65.6 & 0.0912 & 0.0906 & 221 & 1 & 0.0001 \\ 22.5 & 73.8 & 0.1012 & 0.1005 & 224 & 1 & 0.0003 \\ 25.0 & 82.0 & 0.1102 & 0.1104 & 226 & 1 & -0.0005 \\ 27.5 & 90.2 & 0.1205 & 0.1203 & 229 & 1 & -0.0001 \\ 29.0 & 95.1 & 0.1267 & 0.1262 & 230 & 1 & 0.0002\end{array}$

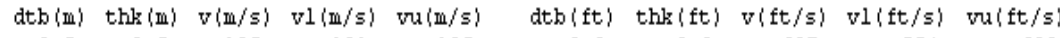

$\begin{array}{rrrrrrrrrr}2.5 & 2.5 & 185 & 181 & 189 & 8.2 & 8.2 & 607 & 594 & 620 \\ 15.0 & 12.5 & 218 & 216 & 220 & 49.2 & 41.0 & 715 & 710 & 721 \\ 29.0 & 14.0 & 253 & 251 & 255 & 95.1 & 45.9 & 830 & 823 & 836\end{array}$

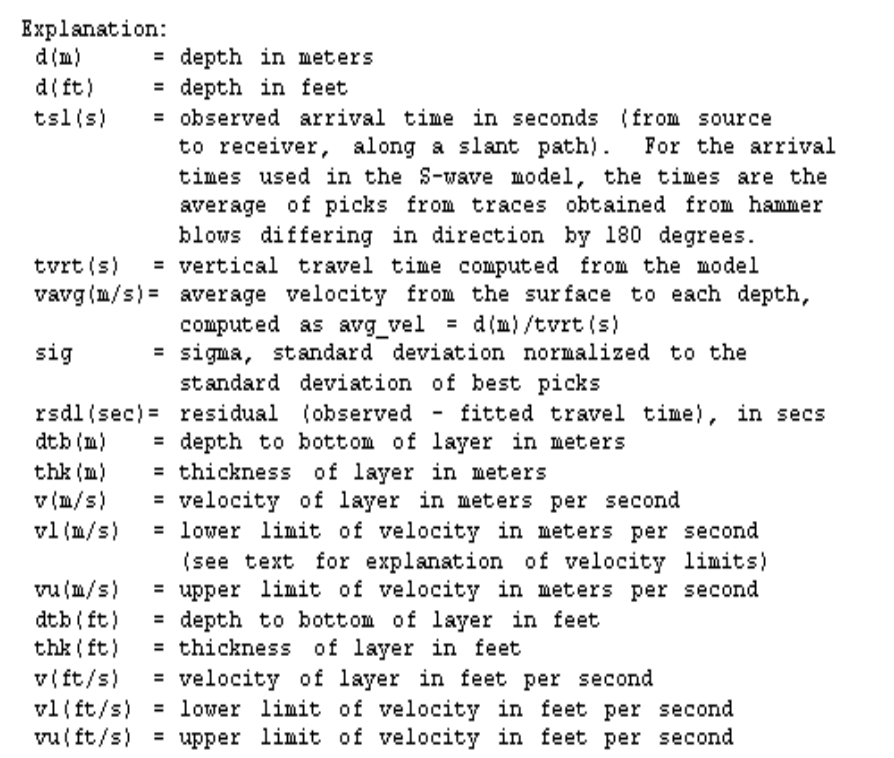


ABLE A-4. P-wave arrival times and velocity summaries.

Location: Physical Sciences Building: P Coordinates: hoffset $=2.00$ travel-time file: $\mathrm{P}: \backslash \mathrm{CPS} \backslash \mathrm{CPSP} . \mathrm{TT}$

$33.88589-118.09700$ Hole_Code: 297 nlayers $=3$

$\begin{array}{rrrrrrr}\mathrm{d}(\mathrm{I}) & \mathrm{d}(\mathrm{ft}) & \mathrm{tsl}(s) & \text { tvrt }(s) & \operatorname{vavg}(\mathrm{m} / \mathrm{s}) & \text { sig } & \mathrm{rsdl}(\mathrm{sec}) \\ 2.5 & 8.2 & 0.0094 & 0.0071 & 353 & 1 & 0.0003 \\ 5.0 & 16.4 & 0.0114 & 0.0119 & 420 & 1 & -0.0014 \\ 7.5 & 24.6 & 0.0194 & 0.0168 & 448 & 1 & 0.0021 \\ 10.0 & 32.8 & 0.0214 & 0.0216 & 463 & 1 & -0.0006 \\ 12.5 & 41.0 & 0.0254 & 0.0264 & 473 & 1 & -0.0014 \\ 15.0 & 49.2 & 0.0310 & 0.0313 & 480 & 1 & -0.0005 \\ 17.5 & 57.4 & 0.0346 & 0.0334 & 524 & 1 & 0.0010 \\ 20.0 & 65.6 & 0.0366 & 0.0355 & 563 & 1 & 0.0009 \\ 22.5 & 73.8 & 0.0380 & 0.0377 & 597 & 1 & 0.0002 \\ 25.0 & 82.0 & 0.0400 & 0.0398 & 628 & 1 & 0.0001 \\ 27.5 & 90.2 & 0.0416 & 0.0419 & 656 & 1 & -0.0004 \\ 29.0 & 95.1 & 0.0430 & 0.0432 & 671 & 1 & -0.0003\end{array}$

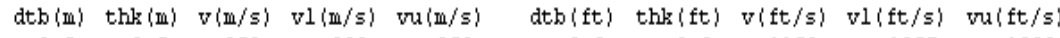

$\begin{array}{rrrrrrrrrr}2.5 & 2.5 & 353 & 322 & 390 & 8.2 & 8.2 & 1158 & 1057 & 1280 \\ 15.0 & 12.5 & 517 & 495 & 540 & 49.2 & 41.0 & 1695 & 1626 & 1771\end{array}$

$\begin{array}{rrrrrrrrrr}15.0 & 12.5 & 517 & 495 & 540 & 49.2 & 41.0 & 1695 & 1626 & 1771 \\ 29.0 & 14.0 & 1172 & 1076 & 1287 & 95.1 & 45.9 & 3845 & 3530 & 4223\end{array}$

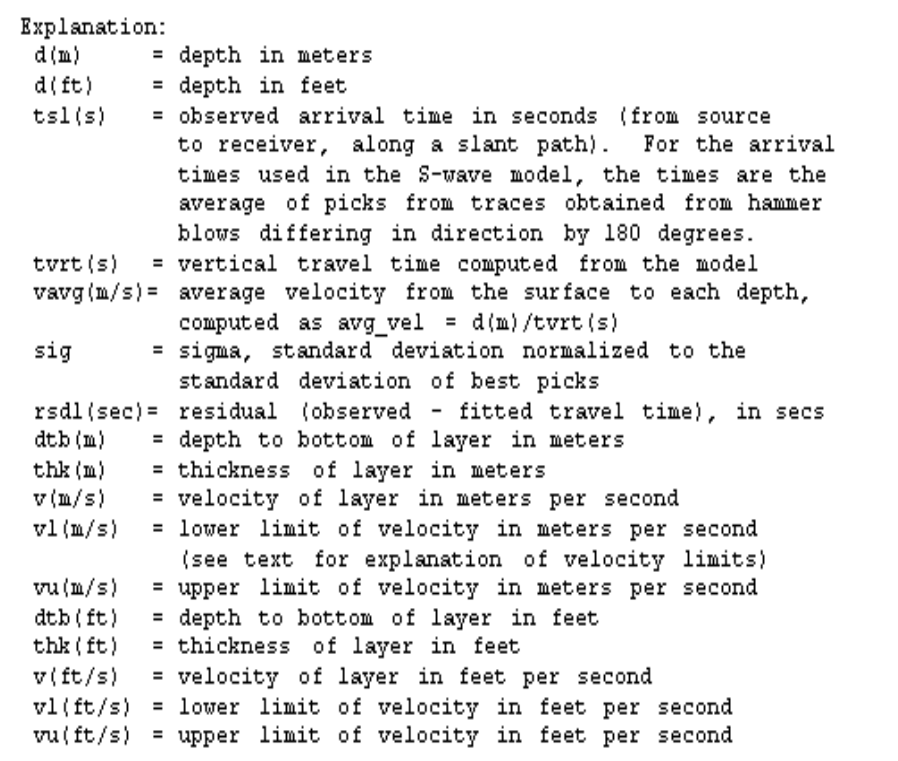




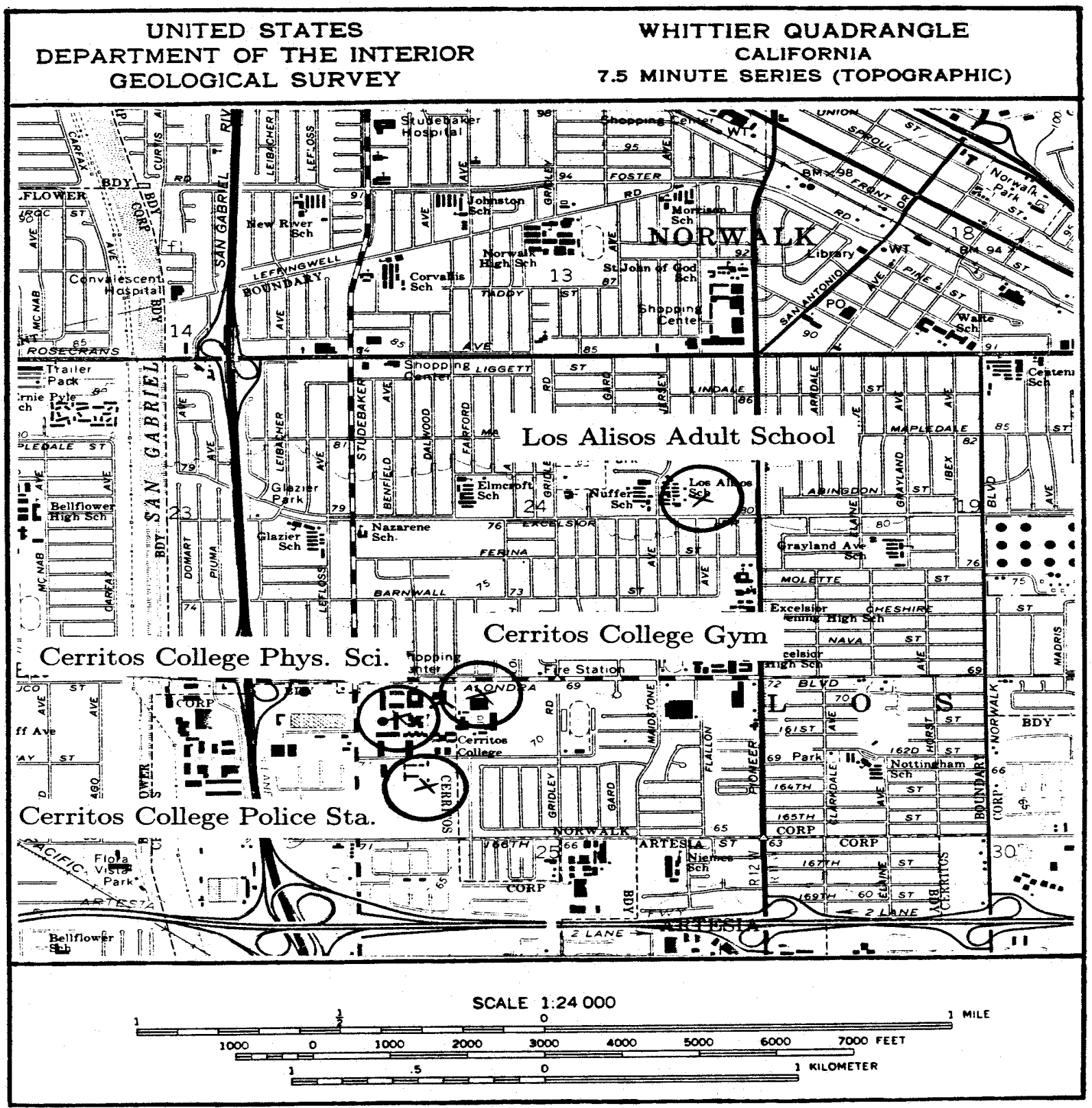

Figure A-11. Site location map for the borehole at Cerritos College Police Building. 


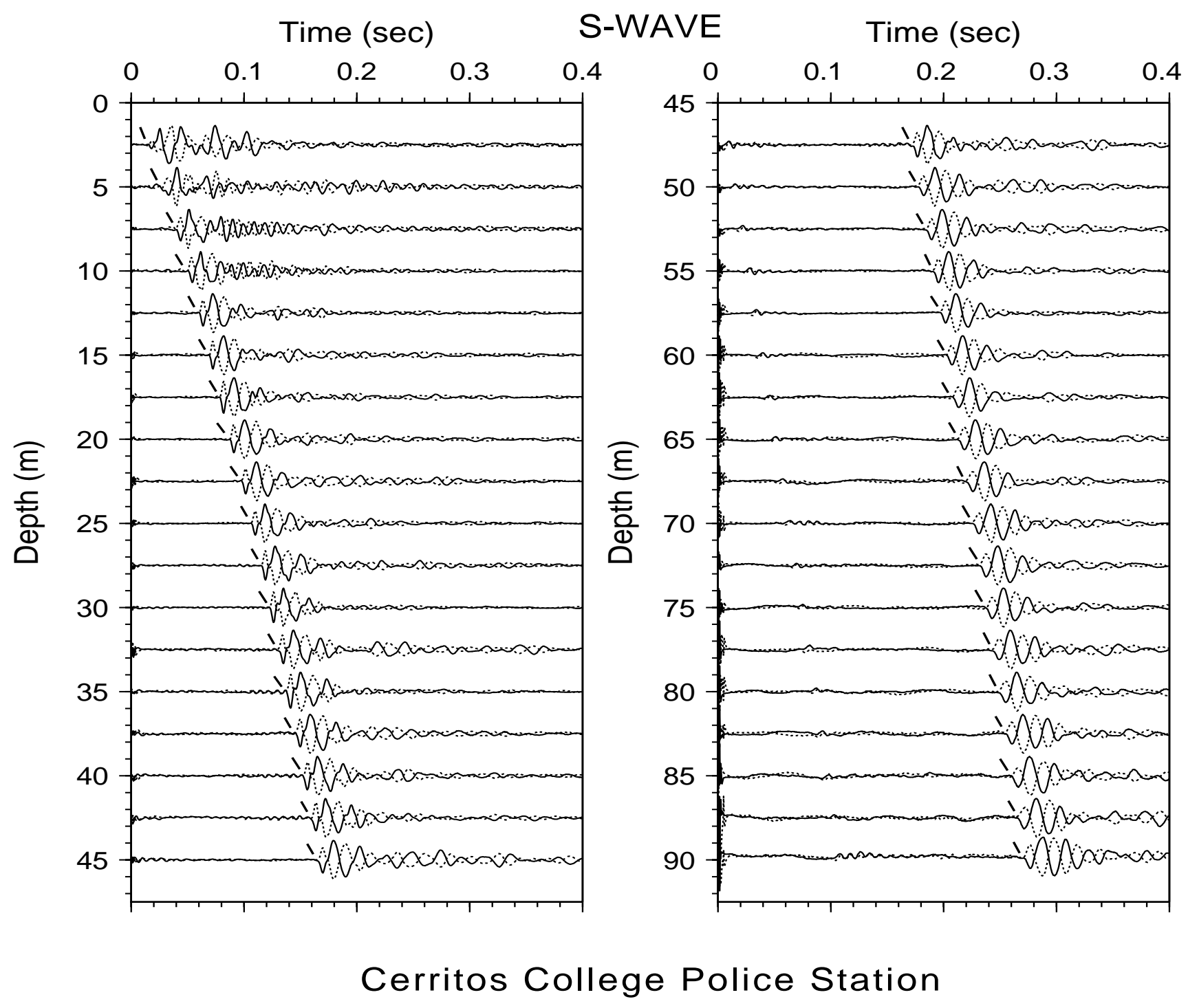

Figure A-12. Horizontal component record section (from impacts in opposite directions) superimposed for identification of S-wave onset. Approximate S-wave time picks are indicated by the hatch marks. 


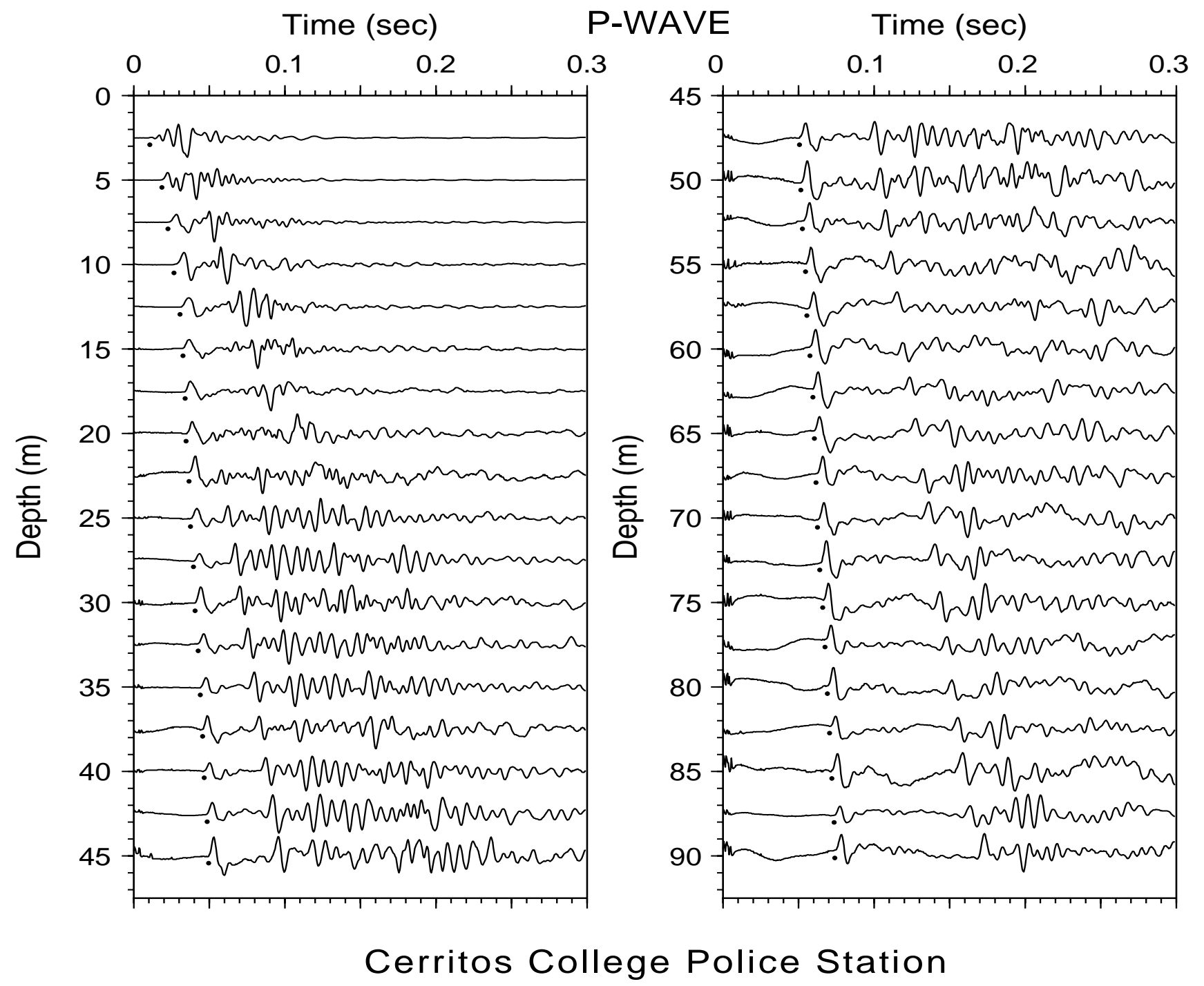

Figure A-13. Vertical component record section. Approximate P-wave arrivals are indicated by the dots. 


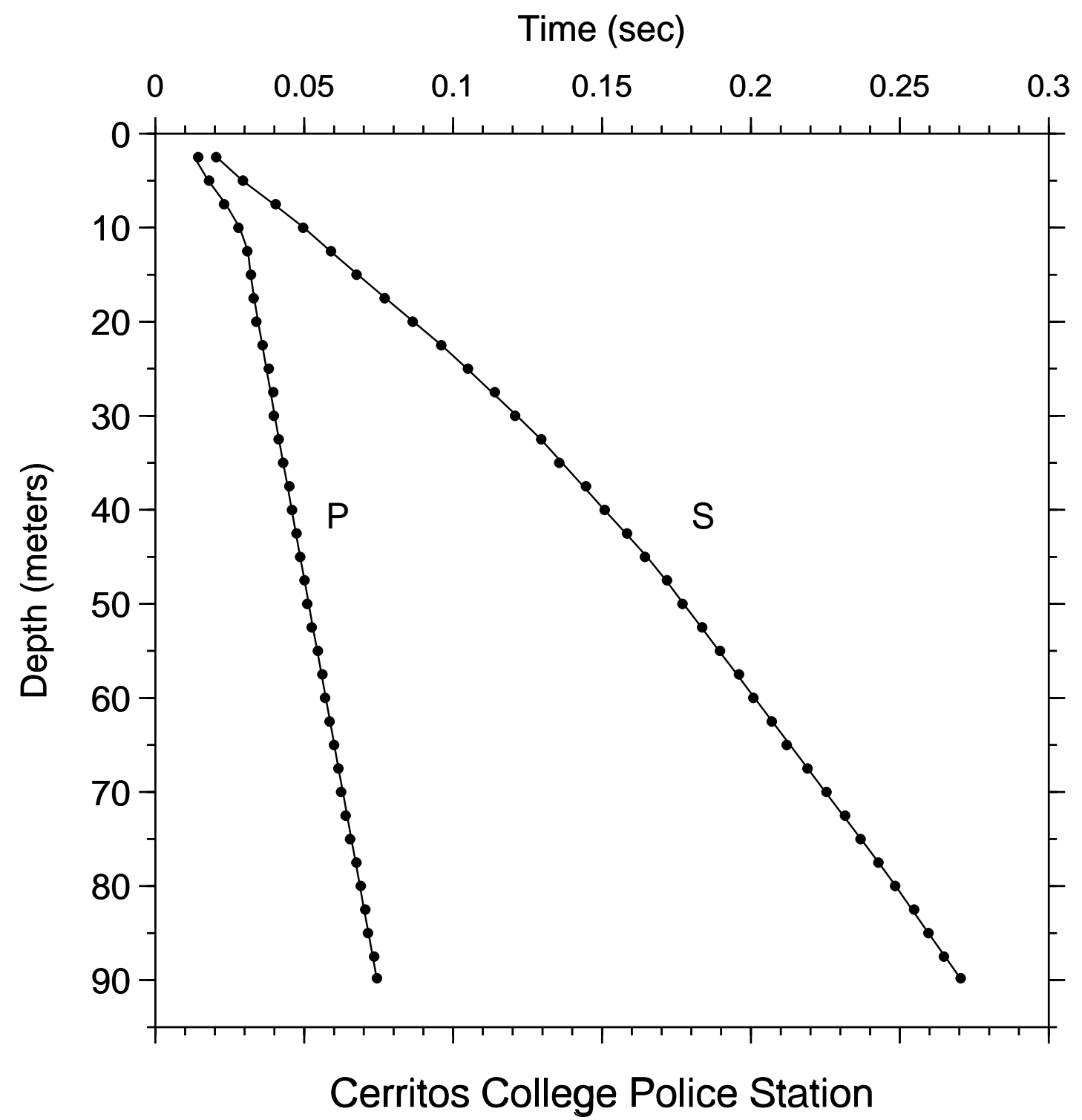

Figure A-14. Time-depth graph of P-wave and S-wave picks. Line segments are straightline interpolations of model predictions at the observation depths. The times for zero depth, not shown, are given by hoffset divided by the velocity in the uppermost layer (see accompanying tables of velocities for specific values). 


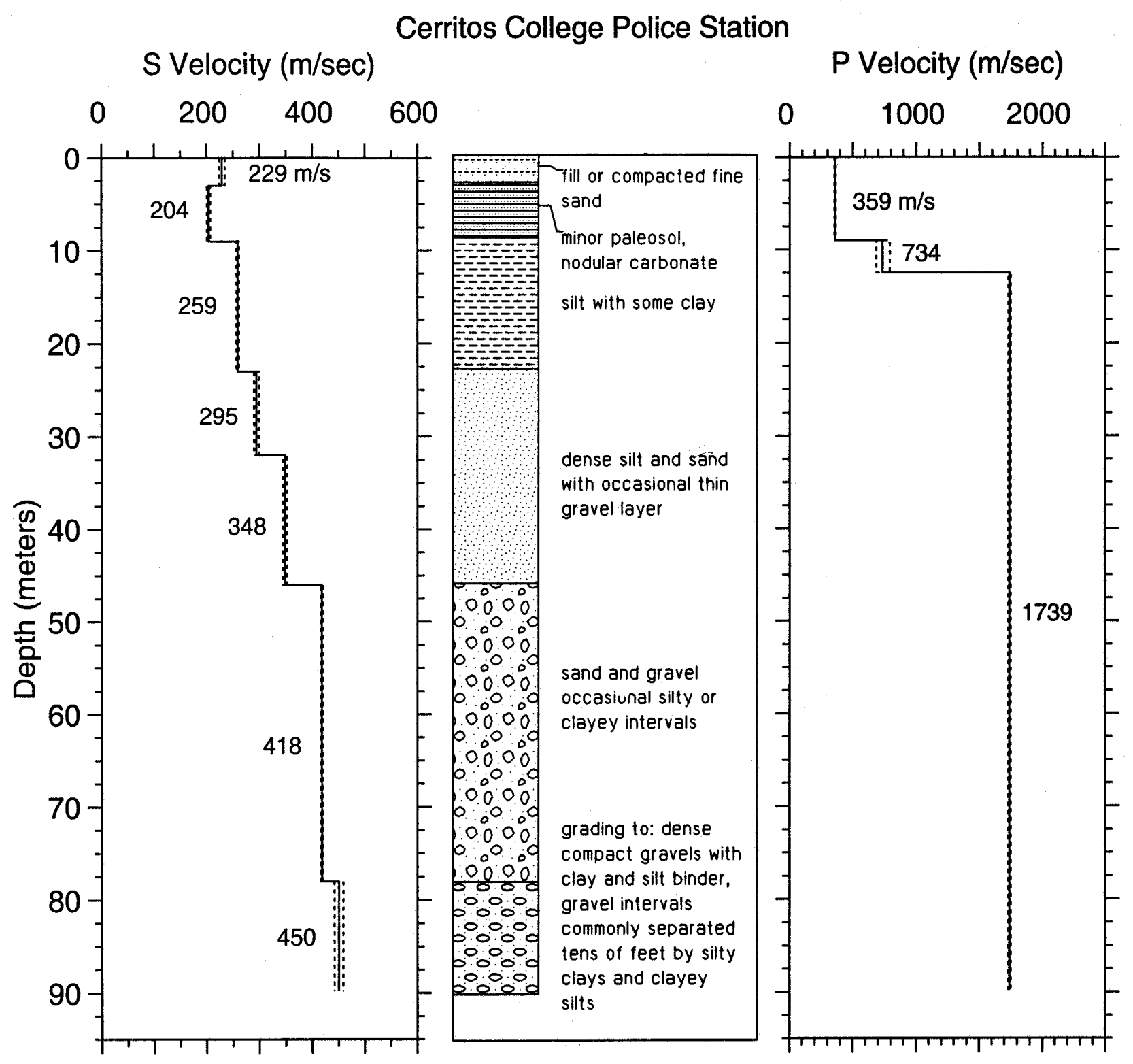

Figure A-15. Velocity profiles for the borehole at Cerritos College Police Building. Generalized geology is shown for correlation with velocities. Dashed lines are one standard deviation. 
ABLB A-5. S-wave arrival times and velocity sumaries.

Location: Cerritos police Building: \& Coordinates: hoffset $=4.00$ travel-time file: $\mathrm{F}: \backslash \mathrm{CPB} \backslash \mathrm{CPBS} . \mathrm{TT}$

\begin{tabular}{|c|c|c|c|c|c|c|}
\hline in) & $d(f t)$ & $t \leq 1(s)$ & Evrt (s) & & sig & \\
\hline & 8.2 & 0.0204 & 0.0109 & 229 & 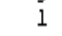 & -0.0002 \\
\hline 5.0 & 16.4 & 0.0294 & 0.0229 & 218 & 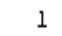 & 0.0001 \\
\hline 7.5 & 24.6 & 0.0404 & 0.0352 & 213 & 1 & 0.0006 \\
\hline 10.0 & 32.8 & 0.0496 & 0.0464 & 216 & 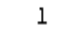 & -0.0003 \\
\hline 12.5 & 41.0 & 0.0590 & 0.0560 & 223 & 1 & 0.0002 \\
\hline 15.0 & 49.2 & 0.0676 & 0.0657 & 228 & 1 & -0.0004 \\
\hline 17.5 & 57.4 & 0.0770 & 0.0753 & 232 & 1 & -0.0003 \\
\hline 0.0 & 65.6 & 0.0864 & 0.0850 & 335 & 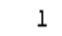 & -0.0003 \\
\hline .5 & 73.8 & 0.0960 & 0.0946 & 238 & 1 & -0.0001 \\
\hline .0 & 82.0 & 0.1050 & 0.1033 & 243 & , & 0.0003 \\
\hline .5 & 90.2 & 0.1140 & 0.1118 & 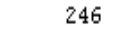 & 1 & 0010 \\
\hline 30.0 & 98.4 & 0.1208 & 0.1203 & 249 & 1 & -0.0006 \\
\hline 32.5 & 106.6 & 0.1296 & 0.1285 & 253 & 1 & 0.0001 \\
\hline 5.0 & 114.8 & 0.1356 & 0.1357 & 258 & 1 & -0.0010 \\
\hline 37.5 & 123.0 & 0.1446 & 0.1429 & 262 & 1 & 0.0009 \\
\hline 40.0 & 131.2 & 0.1509 & 0.1501 & 267 & 1 & 0.0001 \\
\hline 42.5 & 139.4 & 0.1584 & 0.1572 & 270 & 1 & 0.0004 \\
\hline .0 & 147.6 & 0.1644 & 0.1 & 274 & 1 & -0.0007 \\
\hline 7.5 & 155.8 & 0.1718 & 0.1709 & 278 & 1 & 0.0003 \\
\hline 50.0 & 164.0 & 0.1770 & & 283 & 1 & -0.0004 \\
\hline 2.5 & 172.2 & 0.1836 & 0.1829 & 287 & 1 & 0.0002 \\
\hline 55.0 & 180.4 & 0.1896 & 0.1888 & 291 & 1 & 0.0003 \\
\hline 57.5 & 188.6 & 0.1960 & 0.1948 & 295 & 1 & 0.0007 \\
\hline 0.0 & 196.9 & 0.2008 & 0. & 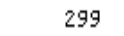 & 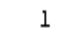 & -0.0004 \\
\hline 52.5 & 205.1 & 0.2070 & 0.2 & 302 & 1 & -0.0002 \\
\hline 65.0 & 213.3 & 0.2120 & 0.2128 & 306 & 1 & -0.0011 \\
\hline 67.5 & 221.5 & 0.2190 & 0.2187 & 309 & 1 & -0.0001 \\
\hline 70.0 & 229.7 & 0.23 & 0.2 & 31. & 1 & 0.0004 \\
\hline 2.5 & 237.9 & 0.23 & 0.2 & & 1 & \\
\hline 5.0 & 246.1 & 0.2368 & 0.2367 & 317 & 1 & -0.0002 \\
\hline 7.5 & 254.3 & & & & & \\
\hline 80.0 & 262.5 & 0.2484 & 0.2483 & 322 & 1 & -0.0002 \\
\hline 82.5 & 270.7 & & & 325 & & \\
\hline & & & & & 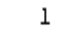 & \\
\hline & 287.1 & 0.2 & 0.2650 & 330 & 1 & \\
\hline & & & & & & 000 \\
\hline
\end{tabular}

$33.88212-118.09680$ Hole_Code: 283

nlayers $=7$

dtb $(\mathrm{II})$ thk $(\mathrm{II}) \mathrm{v}(\mathrm{II} / \mathrm{s}) \mathrm{vl}(\mathrm{II} / \mathrm{s}) \mathrm{vu}(\mathrm{II} / \mathrm{s})$ $\begin{array}{lllll}3.0 & 3.0 & 229 & 224 & 235\end{array}$ $\begin{array}{rrr}9.0 & 6.0 & 204\end{array}$ $295-290-262$ $\begin{array}{lllll}46.0 & 14.0 & 348 & 345 & 352\end{array}$

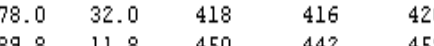
$23.0 \quad 14.0 \quad 259$

$\mathrm{dtb}(\mathrm{ft}) \quad \mathrm{thl}(\mathrm{ft}) \quad \mathrm{v}(\mathrm{ft} / \mathrm{s}) \quad \mathrm{vl}(\mathrm{ft} / \mathrm{s}) \quad \mathrm{vu}(\mathrm{ft} / \mathrm{s})$ $\begin{array}{rrrrr}9.8 & 9.8 & 752 & 735 & 770\end{array}$ $\begin{array}{lllll}29.5 & 19.7 & 669 & 658 & 680\end{array}$ $\begin{array}{rrrrr}75.5 & 45.9 & 849 & 841 & 858 \\ 105.0 & 29.5 & 966 & 951 & 982\end{array}$

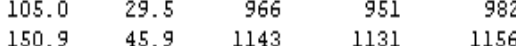
$\begin{array}{rrrrr}150.9 & 45.9 & 1143 & 1131 & 1156 \\ 255.9 & 105.0 & 1372 & 1365 & 1380\end{array}$ $\begin{array}{rrrrr}255.9 & 105.0 & 1372 & 1365 & 1380 \\ 294.6 & 38.7 & 1477 & 1450 & 1505\end{array}$

\author{
Bxplanation: \\ $d(i \mathrm{i})=$ depth in meters \\ $\mathrm{d}(\mathrm{ft})=$ depth in feet \\ tsl(s) = observed arrival time in seconds (from source \\ to receiver, along a slant path). For the arrival \\ times used in the $\$$-wave model, the times are the \\ average of picks from traces obtained from hamer \\ blows differing in direction by 180 degrees. \\ tvrt $(s)=$ vertical travel time computed from the model \\ computed as avg vel $=d(i$ i $) /$ tvrt $\{s\rangle$ \\ sig = sigma, standard deviation normalized to the \\ $\begin{aligned}= & \text { sigma, standard deviation normaliz } \\ & \text { standard deviation of best picks }\end{aligned}$ \\ rsdl (sec) = residual (observed - fitted travel time), in secs \\ $d t b(\mathbb{i})=$ depth to bottom of layer in meters \\ thl $(\mathrm{II}) \quad=$ thickness of layer in meters \\ $\mathrm{v}(\mathrm{i} / \mathrm{s})=$ velocity of layer in meters per second \\ $\mathrm{vl}(\mathrm{m} / \mathrm{s})=$ lower limit of velocity in meters per second \\ (see text for explanation of velocity limits) \\ $\mathrm{vu}(\mathrm{i} / \mathrm{s})=$ upper limit of velocity in meters per second \\ $\mathrm{dtb}(\mathrm{ft})=$ depth to bottom of layer in feet \\ thl $(\mathrm{ft})=$ thickness of layer in feet \\ $\mathrm{v}(\mathrm{ft} / \mathrm{s})=$ velocity of layer in feet per second \\ $\mathrm{vl}(\mathrm{ft} / \mathrm{s})=$ lower limit of velocity in feet per second \\ $\mathrm{vu}(\mathrm{ft} / \mathrm{s})=$ upper limit of velocity in feet per second
}


ABLB $4-6$. P-wave arrival times and velocity sumaries.

Location: Cerritos police Building: P Coordinates: hoffset $=4.00$ travel-time file: $\mathrm{F}: \backslash \mathrm{CPB} \backslash \mathrm{CPBP} . \mathrm{TT}$

$\begin{array}{rrrrrrr}d(\mathrm{~m}) & d(\mathrm{ft}) & \mathrm{tsl}(s) & \text { tvrt }(s) & \text { vavg }(\mathrm{II} / s) & \text { sig } & \mathrm{rsdl}(\mathrm{sec}) \\ 2.5 & 8.2 & 0.0144 & 0.0070 & 359 & 1 & 0.0013 \\ 5.0 & 16.4 & 0.0180 & 0.0139 & 359 & 1 & 0.0002 \\ 7.5 & 24.6 & 0.0231 & 0.0209 & 359 & 1 & -0.0006 \\ 10.0 & 32.8 & 0.0279 & 0.0264 & 378 & 1 & -0.0004 \\ 12.5 & 41.0 & 0.0309 & 0.0298 & 419 & 1 & -0.0002 \\ 15.0 & 49.2 & 0.0321 & 0.0313 & 480 & 1 & 0.0001 \\ 17.5 & 57.4 & 0.0330 & 0.0327 & 535 & 1 & -0.0002 \\ 20.0 & 65.6 & 0.0339 & 0.0342 & 586 & 1 & -0.0006 \\ 22.5 & 73.8 & 0.0360 & 0.0356 & 632 & 1 & 0.0001 \\ 25.0 & 82.0 & 0.0381 & 0.0370 & 675 & 1 & 0.0008 \\ 27.5 & 90.2 & 0.0396 & 0.0385 & 715 & 1 & 0.0009 \\ 30.0 & 98.4 & 0.0398 & 0.0399 & 752 & 1 & -0.0003 \\ 32.5 & 106.6 & 0.0414 & 0.0413 & 786 & 1 & -0.0001 \\ 35.0 & 114.8 & 0.0429 & 0.0428 & 818 & 1 & 0.0000 \\ 37.5 & 123.0 & 0.0450 & 0.0442 & 848 & 1 & 0.0006 \\ 40.0 & 131.2 & 0.0459 & 0.0457 & 876 & 1 & 0.0001 \\ 42.5 & 139.4 & 0.0474 & 0.0471 & 903 & 1 & 0.0002 \\ 45.0 & 147.6 & 0.0486 & 0.0485 & 927 & 1 & 0.0000 \\ 47.5 & 155.8 & 0.0501 & 0.0500 & 951 & 1 & 0.0000 \\ 50.0 & 164.0 & 0.0510 & 0.0514 & 973 & 1 & -0.0005 \\ 52.5 & 172.2 & 0.0525 & 0.0528 & 994 & 1 & -0.0004 \\ 55.0 & 180.4 & 0.0546 & 0.0543 & 1013 & 1 & 0.0002 \\ 57.5 & 188.6 & 0.0561 & 0.0557 & 1032 & 1 & 0.0003 \\ 60.0 & 196.9 & 0.0570 & 0.0572 & 1050 & 1 & -0.0002 \\ 62.5 & 205.1 & 0.0585 & 0.0586 & 1067 & 1 & -0.0002 \\ 65.0 & 213.3 & 0.0600 & 0.0600 & 1083 & 1 & -0.0001 \\ 67.5 & 221.5 & 0.0615 & 0.0615 & 1098 & 1 & 0.0000 \\ 70.0 & 229.7 & 0.0624 & 0.0629 & 1113 & 1 & -0.0006 \\ 72.5 & 237.9 & 0.0639 & 0.0643 & 1127 & 1 & -0.0005 \\ 75.0 & 246.1 & 0.0654 & 0.0658 & 1140 & 1 & -0.0004 \\ 77.5 & 254.3 & 0.0675 & 0.0672 & 1153 & 1 & 0.0002 \\ 80.0 & 262.5 & 0.0690 & 0.0687 & 1165 & 1 & 0.0003 \\ 82.5 & 270.7 & 0.0705 & 0.0701 & 1177 & 1 & 0.0004 \\ 85.0 & 278.9 & 0.0714 & 0.0715 & 1188 & 1 & -0.0002 \\ 87.5 & 287.1 & 0.0735 & 0.0730 & 1199 & 1 & 0.0005 \\ 89.8 & 294.6 & 0.0744 & 0.0743 & 1209 & 1 & 0.0001\end{array}$

$33.88212-118.09680$ Hole_Code: 283 nlayers $=3$

dtb (II) $\operatorname{thl}(\mathrm{II}) \quad \mathrm{v}(\mathrm{II} / \mathrm{s}) \quad \mathrm{vl}(\mathrm{II} / \mathrm{s}) \quad \mathrm{vu}(\mathrm{II} / \mathrm{s})$ $\begin{array}{lllll}9.0 & 9.0 & 359 & 355 & 364\end{array}$ $\begin{array}{rrrrr}12.5 & 3.5 & 734 & 685 & 792\end{array}$ $\begin{array}{lll}89.8 & 77.3 & 1739\end{array}$ dtb (ft) thk $(\mathrm{ft}) \quad \mathrm{v}(\mathrm{ft} / \mathrm{s}) \quad \mathrm{vl}(\mathrm{ft} / \mathrm{s}) \quad \mathrm{vu}(\mathrm{ft} / \mathrm{s})$ $\begin{array}{lllll}29.5 & 29.5 & 1179 & 1164 & 1193 \\ 41.0 & 11.5 & 2410 & 2247 & 2598\end{array}$ $294.6-253.6 \quad 5705 \quad 5671-5738$

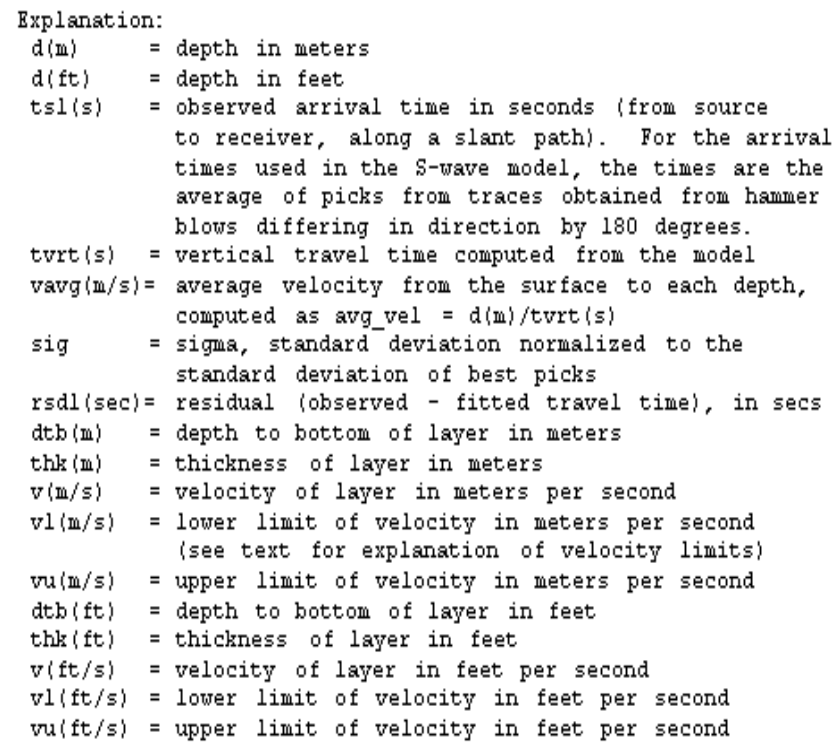




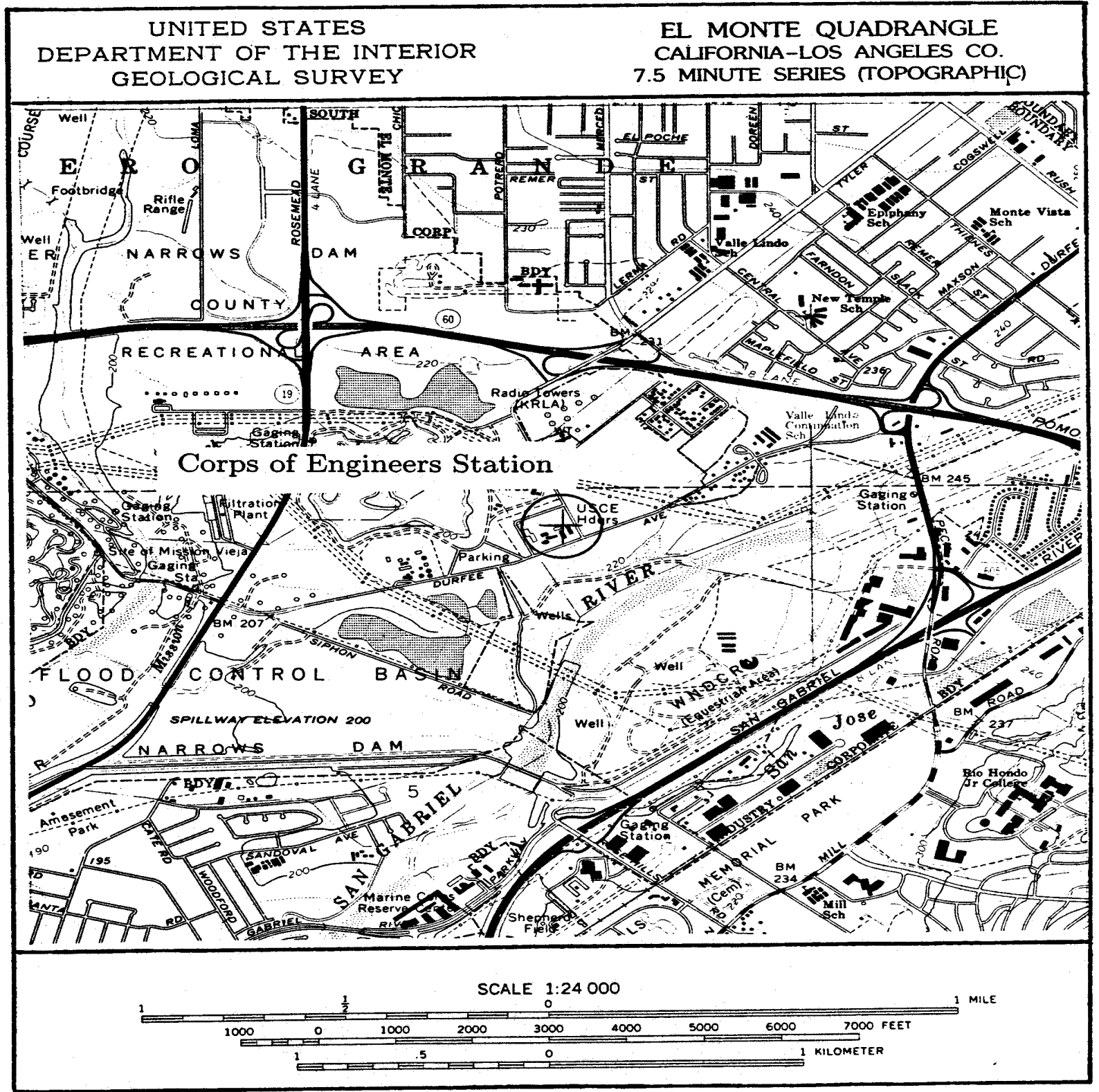

Figure A-16. Site location map for the borehole at Corps of Engineers Station. The accelerograph is located approximately 45 meters from the borehole. 


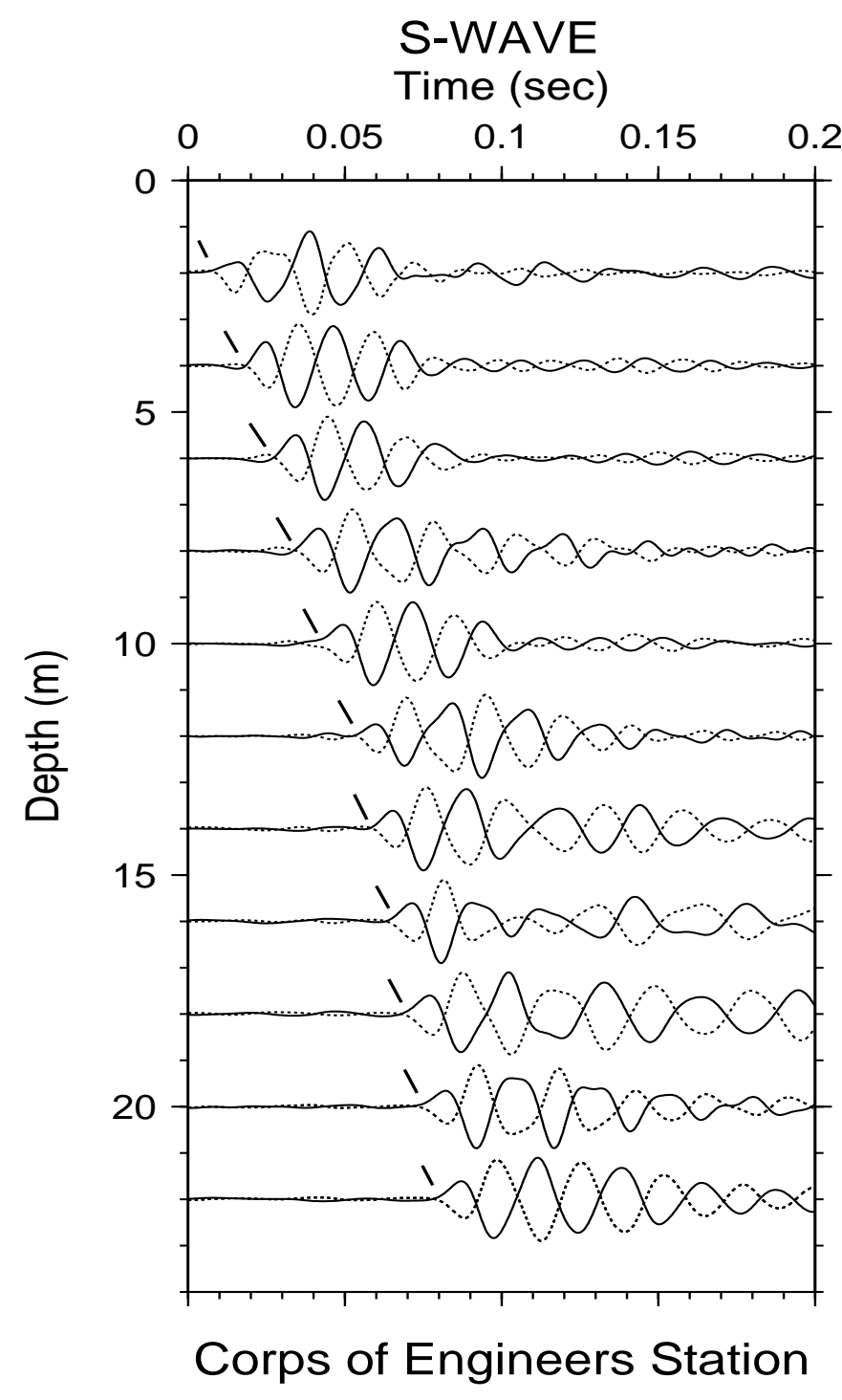

Figure A-17. Horizontal component record section (from impacts in opposite directions) superimposed for identification of S-wave onset. Approximate S-wave time picks are indicated by the hatch marks. 


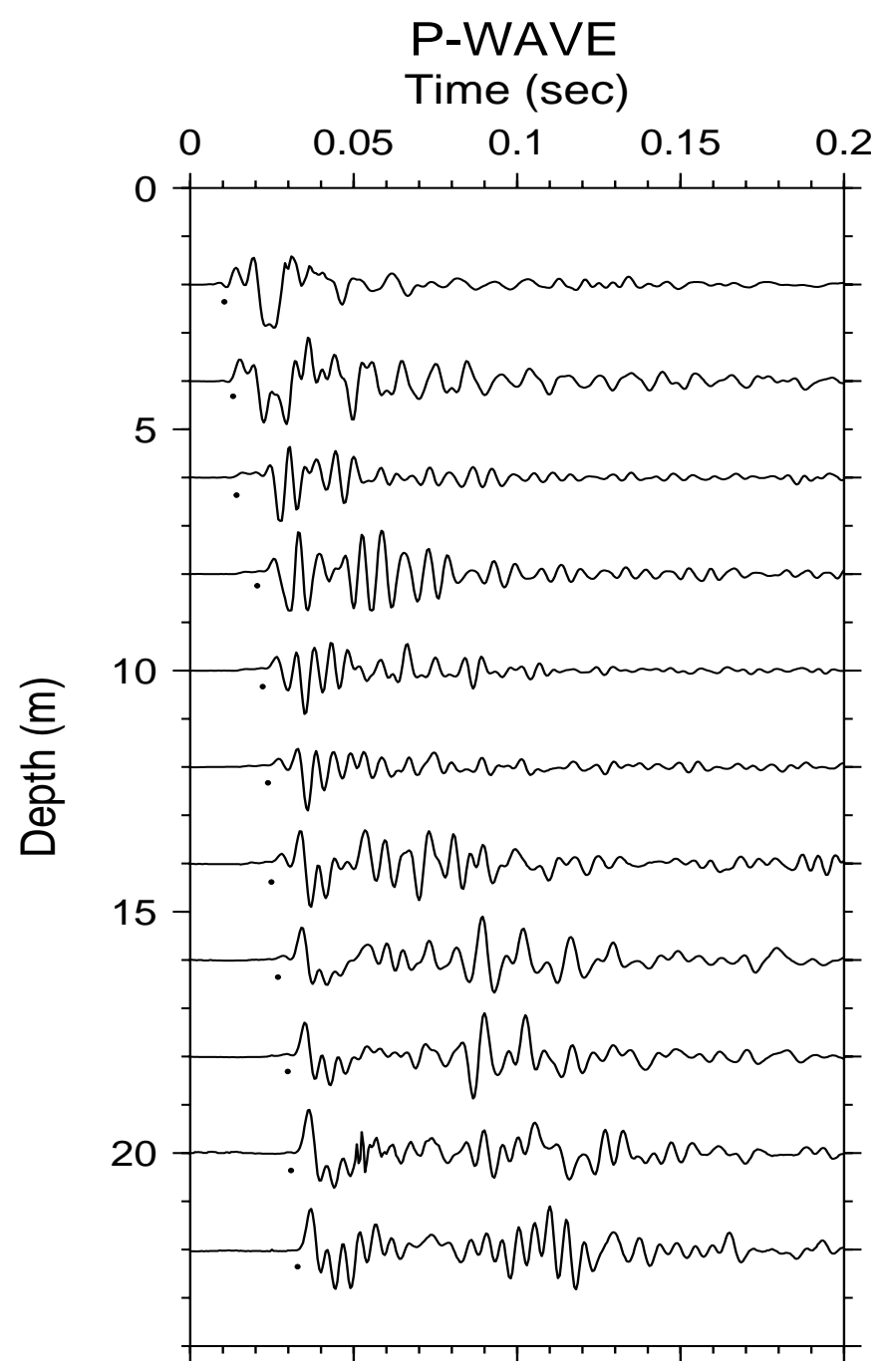

Corps of Engineers Station

Figure A-18. Vertical component record section. Approximate P-wave arrivals are indicated by the dots. 


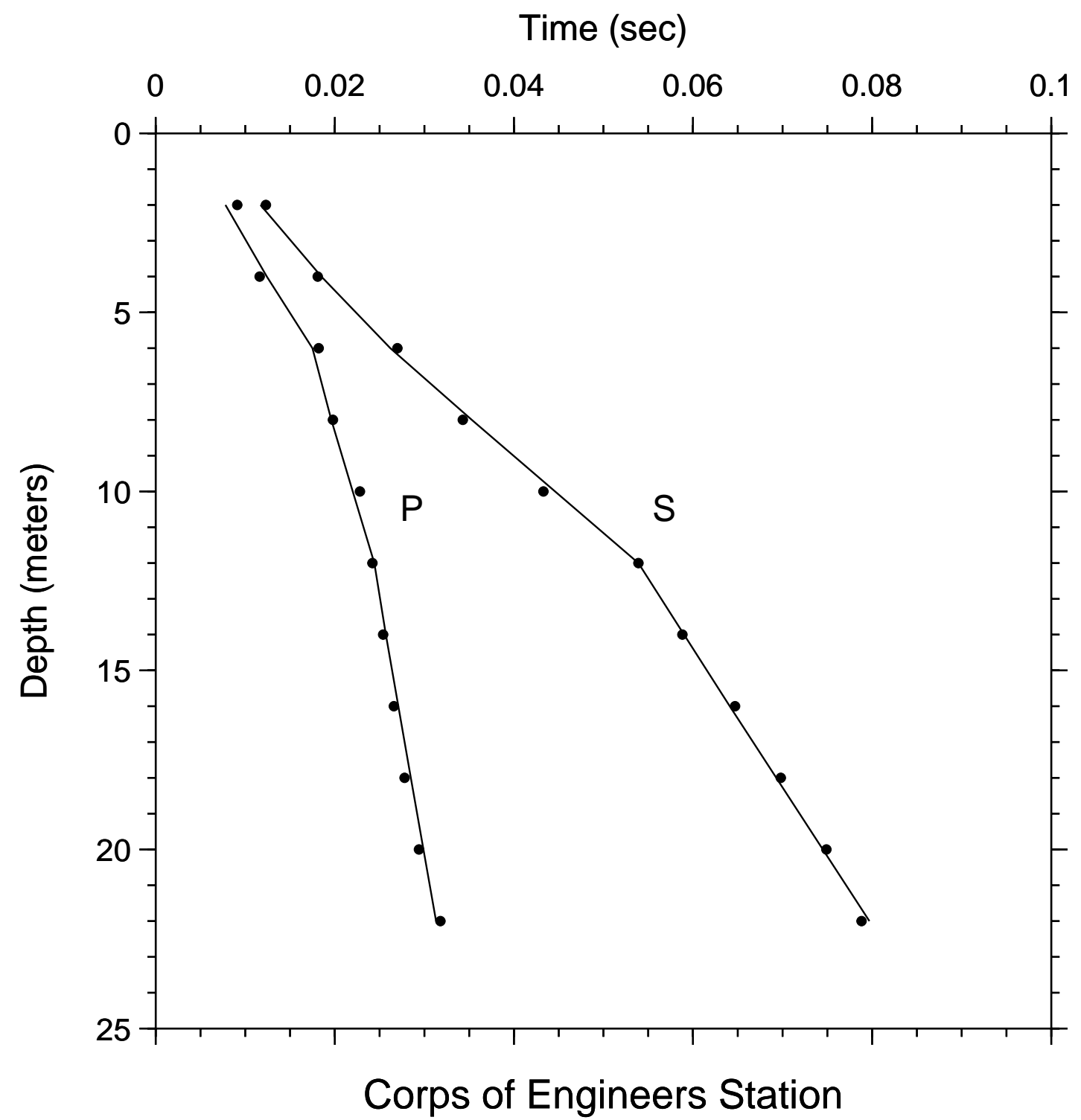

Figure A-19. Time-depth graph of P-wave and S-wave picks. Line segments are straightline interpolations of model predictions at the observation depths. The times for zero depth, not shown, are given by hoffset divided by the velocity in the uppermost layer (see accompanying tables of velocities for specific values). 


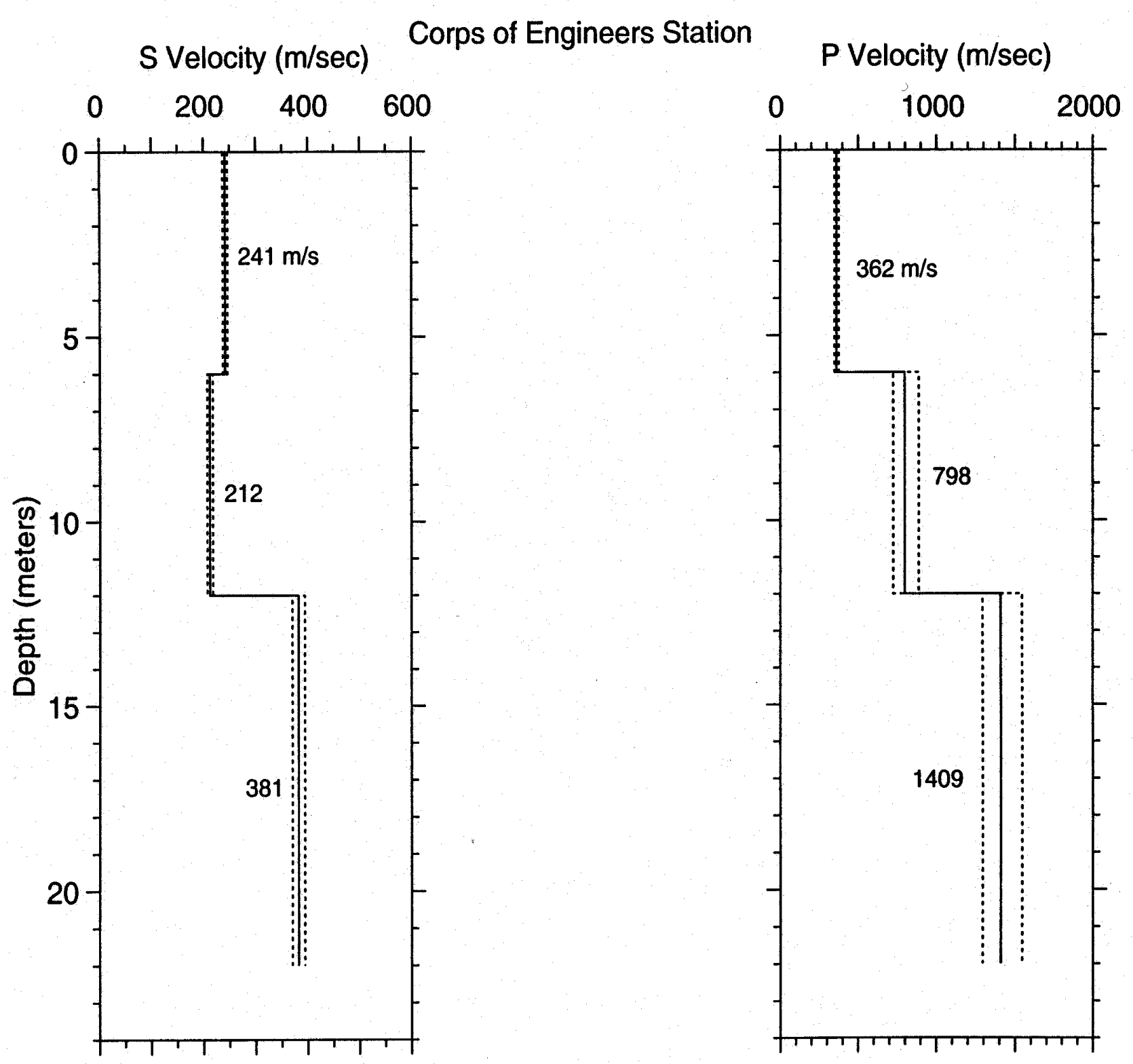

Figure A-20. S- and P-wave velocity profiles with dashed lines representing one standard deviation. Lithology is not available from this borehole. 
ABLE A-7. S-wave arrival times and velocity sumaries.

Location: Corps of Bngineers Station: $S$ Coordinates: hoffset $=2.00$ travel-time file: $\mathrm{F}: \backslash$ MAR $\backslash$ NARS.TT

$34.03219-118.05225$ Hole_Code: 298

nlayers $=3$

$\begin{array}{rrrrcrr}d(\mathbb{I}) & d(f t) & t s l(s) & t v r t(s) & \text { vavg }(\mathrm{m} / s) & \text { sig } & r s d l(s e c) \\ 2.0 & 6.6 & 0.0123 & 0.0083 & 241 & 1 & 0.0006 \\ 4.0 & 13.1 & 0.0181 & 0.0166 & 241 & 1 & -0.0004 \\ 6.0 & 19.7 & 0.0270 & 0.0249 & 241 & 1 & 0.0008 \\ 8.0 & 26.2 & 0.0343 & 0.0343 & 233 & 1 & -0.0010 \\ 10.0 & 32.8 & 0.0433 & 0.0438 & 228 & 2 & -0.0012 \\ 12.0 & 39.4 & 0.0539 & 0.0532 & 226 & 1 & 0.0000 \\ 14.0 & 45.9 & 0.0588 & 0.0584 & 240 & 1 & -0.0002 \\ 16.0 & 52.5 & 0.0647 & 0.0637 & 251 & 1 & 0.0006 \\ 18.0 & 59.1 & 0.0698 & 0.0689 & 261 & 1 & 0.0005 \\ 20.0 & 65.6 & 0.0749 & 0.0742 & 270 & 1 & 0.0004 \\ 22.0 & 72.2 & 0.0788 & 0.0794 & 277 & 1 & -0.0009\end{array}$

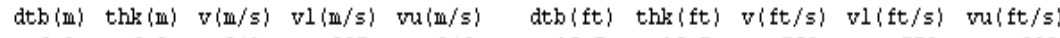
$\begin{array}{rrrrrrrrrr}6.0 & 6.0 & 241 & 237 & 246 & 19.7 & 19.7 & 792 & 778 & 808\end{array}$

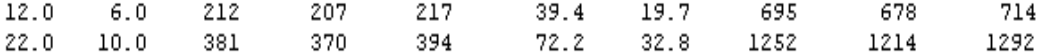


ABLE A-8. p-wave arrival times and velocity sumaries.

Location: Corps of Engineers Station: $p$ Coordinates: hoffset $=2.00$ travel-time file: $\mathrm{P}:\lfloor$ NMR $\backslash$ NMRP.TT

$34.03219-118.05225$ Hole_Code: 298 nlayers $=3$

\begin{tabular}{|c|c|c|c|c|c|c|}
\hline Ii)\} & $\mathrm{d}(\mathrm{ft})$ & $1(s)$ & $\operatorname{tvrt}(s)$ & $v$ & sig & $5 \mathrm{dl} / \mathrm{s}$ \\
\hline 2.0 & 6.6 & 0.0091 & 0.0055 & 362 & 2 & 0.0012 \\
\hline 4.0 & 13.1 & 0.0116 & 0.0110 & 362 & 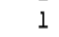 & -0.0007 \\
\hline 6.0 & 19.7 & .0182 & 0.0166 & 362 & & 0.0008 \\
\hline 8.0 & 26.2 & 0. & 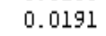 & 415 & & 0.0002 \\
\hline .0 & 32.8 & 0228 & 0.02 & 463 & & 0.0009 \\
\hline & 39 & 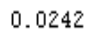 & & 498 & & \\
\hline .0 & 45.9 & 02 & 0.02 & 54 & & -0 . \\
\hline 5.0 & 52.5 & 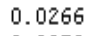 & & 0 & & \\
\hline .0 & 591 & 8 & 4 & 60 & & -0 . \\
\hline 20.0 & 65.6 & 0.0294 & 0.0298 & 672 & & -0.0004 \\
\hline 2.0 & 72.2 & 0.0318 & 0.0312 & 705 & & 0.0005 \\
\hline
\end{tabular}

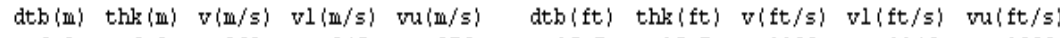

$\begin{array}{rrrrrrrrrr}6.0 & 6.0 & 362 & 349 & 376 & 19.7 & 19.7 & 1188 & 1146 & 1233 \\ 12.0 & 6.0 & 798 & 724 & 889 & 39.4 & 19.7 & 2618 & 2375 & 2915 \\ 22.0 & 10.0 & 1409 & 1294 & 1545 & 72.2 & 32.8 & 4622 & 4246 & 5070\end{array}$

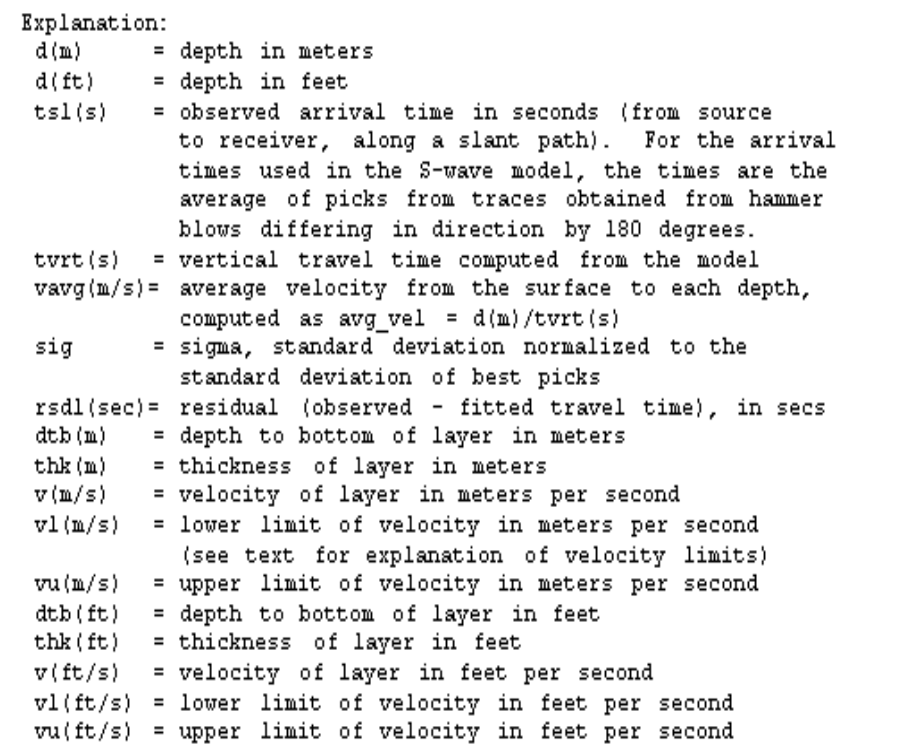






Figure A-21. Site location map for the borehole at Hoover School. The accelerograph is located approximately 30 meters from the borehole. 


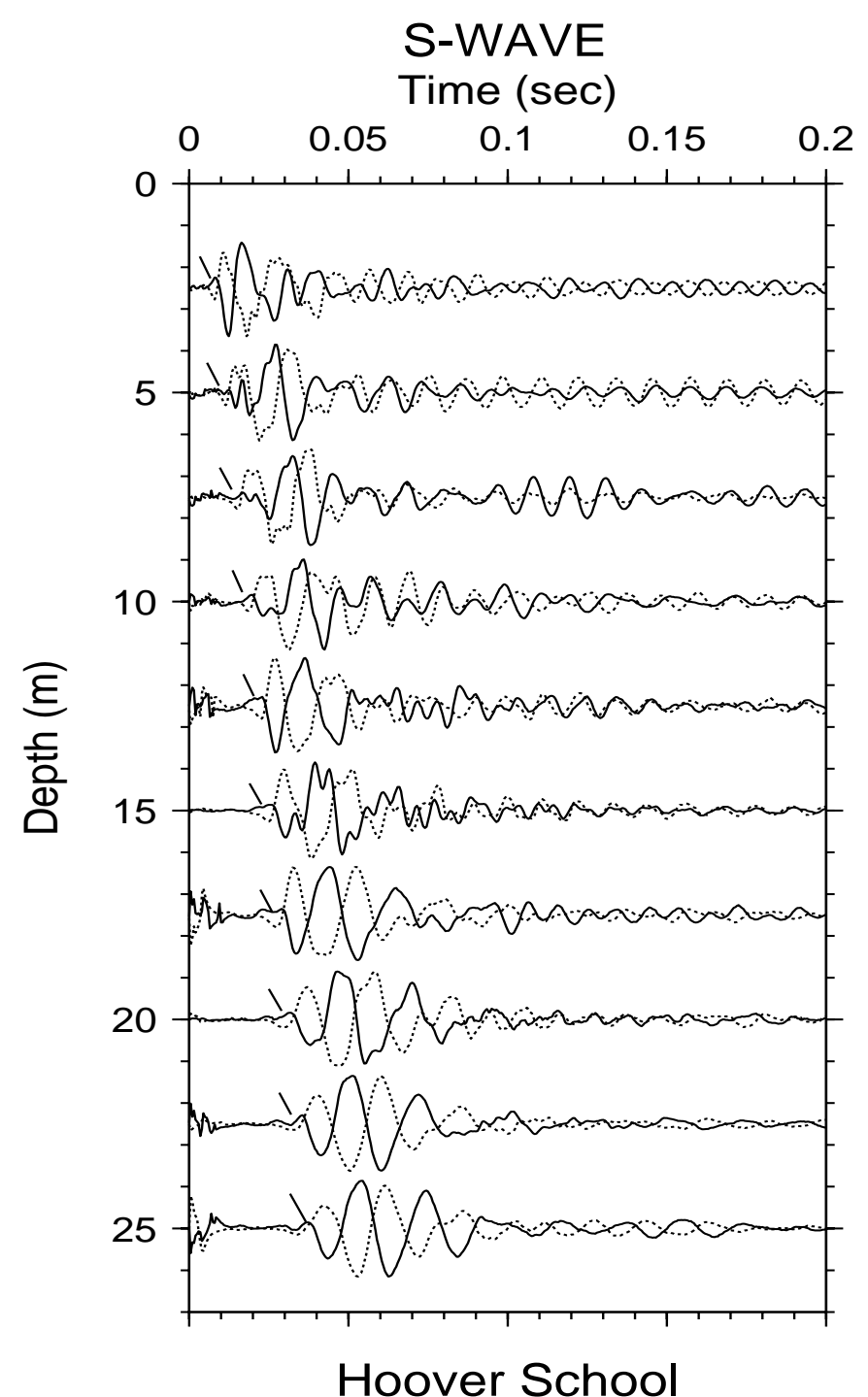

Figure A-22. Horizontal component record section (from impacts in opposite directions) superimposed for identification of S-wave onset. Approximate S-wave time picks are indicated by the hatch marks. 


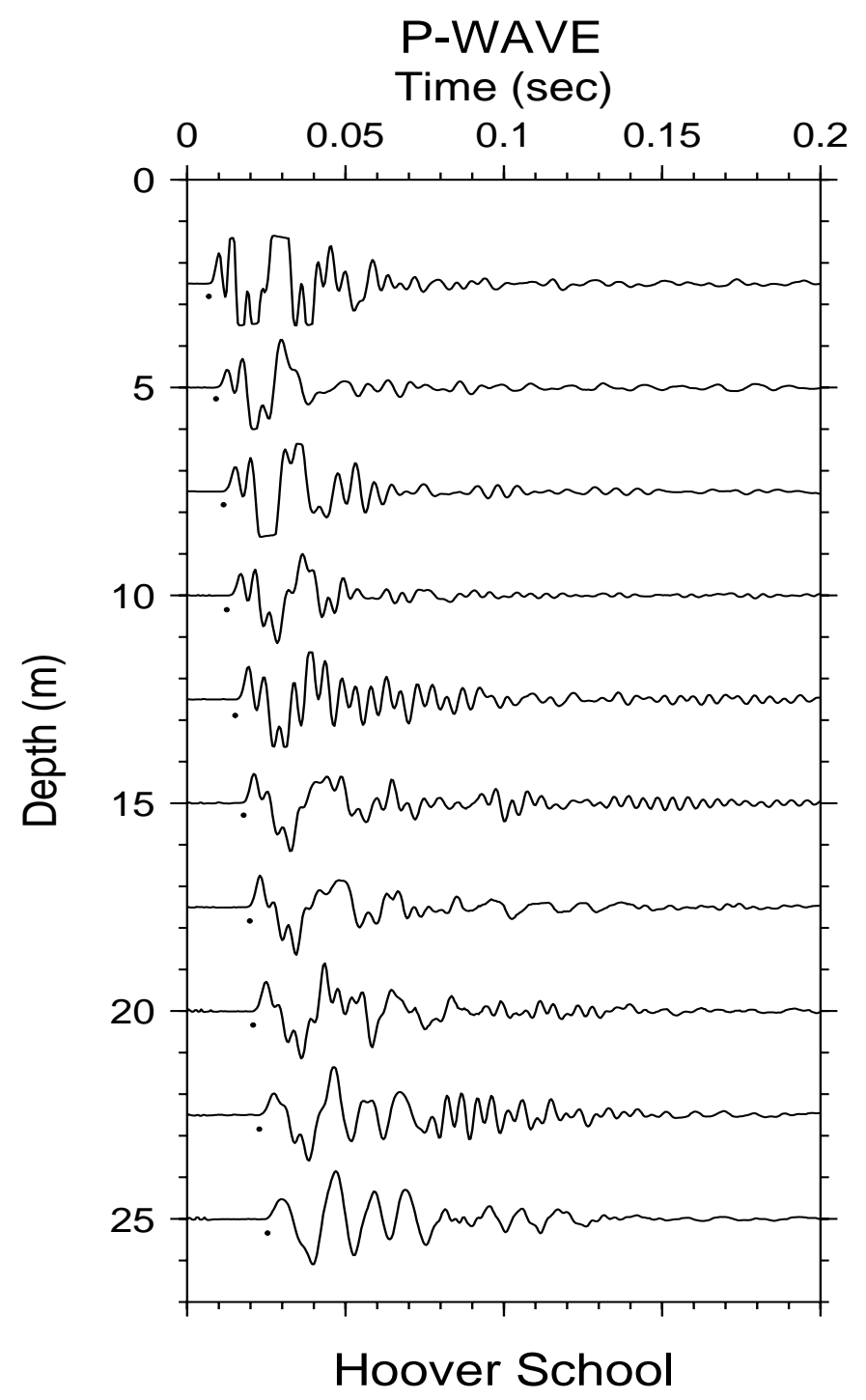

Figure A-23. Vertical component record section. Approximate P-wave arrivals are indicated by the dots. 


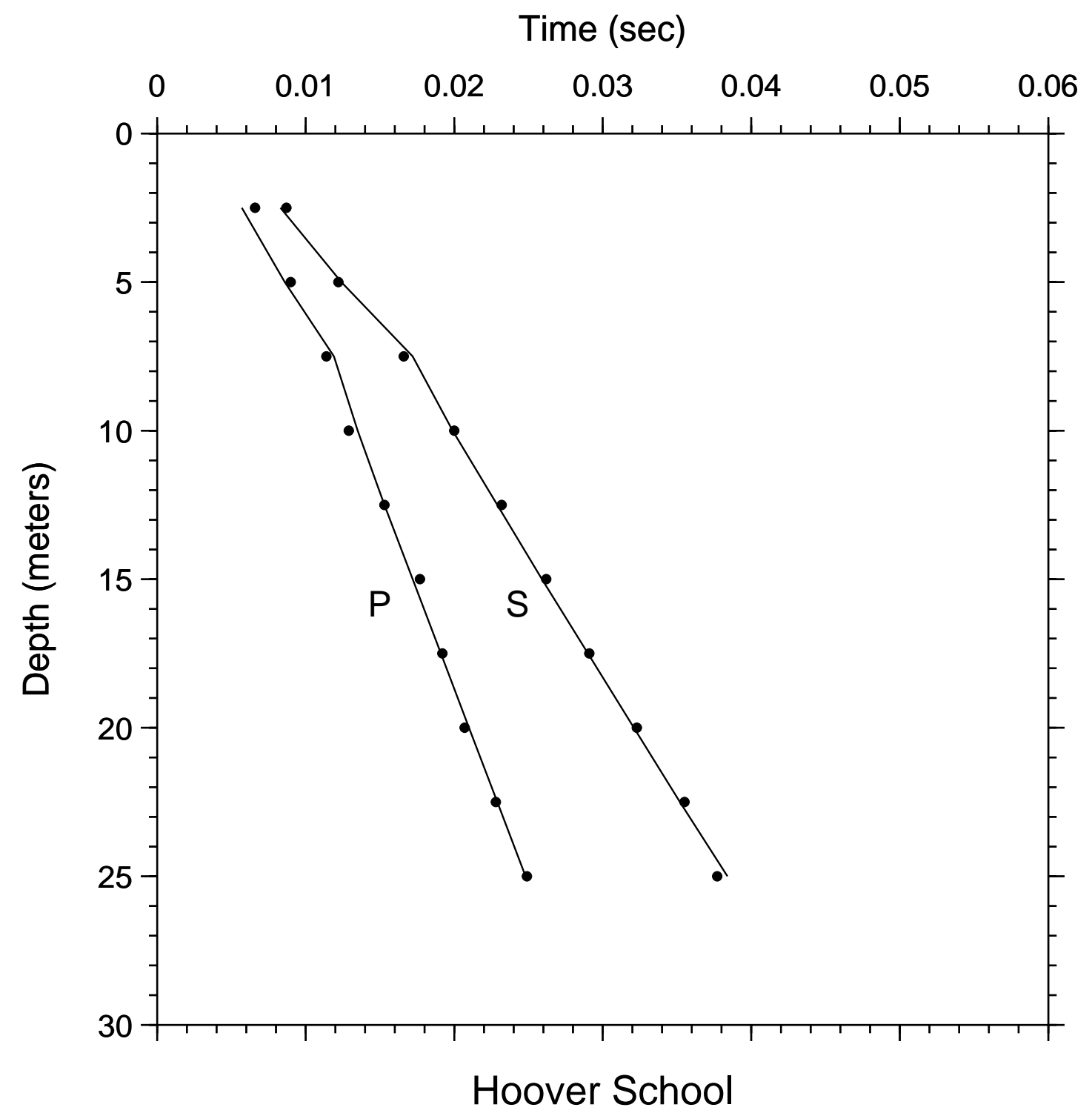

Figure A-24. Time-depth graph of P-wave and S-wave picks. Line segments are straightline interpolations of model predictions at the observation depths. The times for zero depth, not shown, are given by hoffset divided by the velocity in the uppermost layer (see accompanying tables of velocities for specific values). 


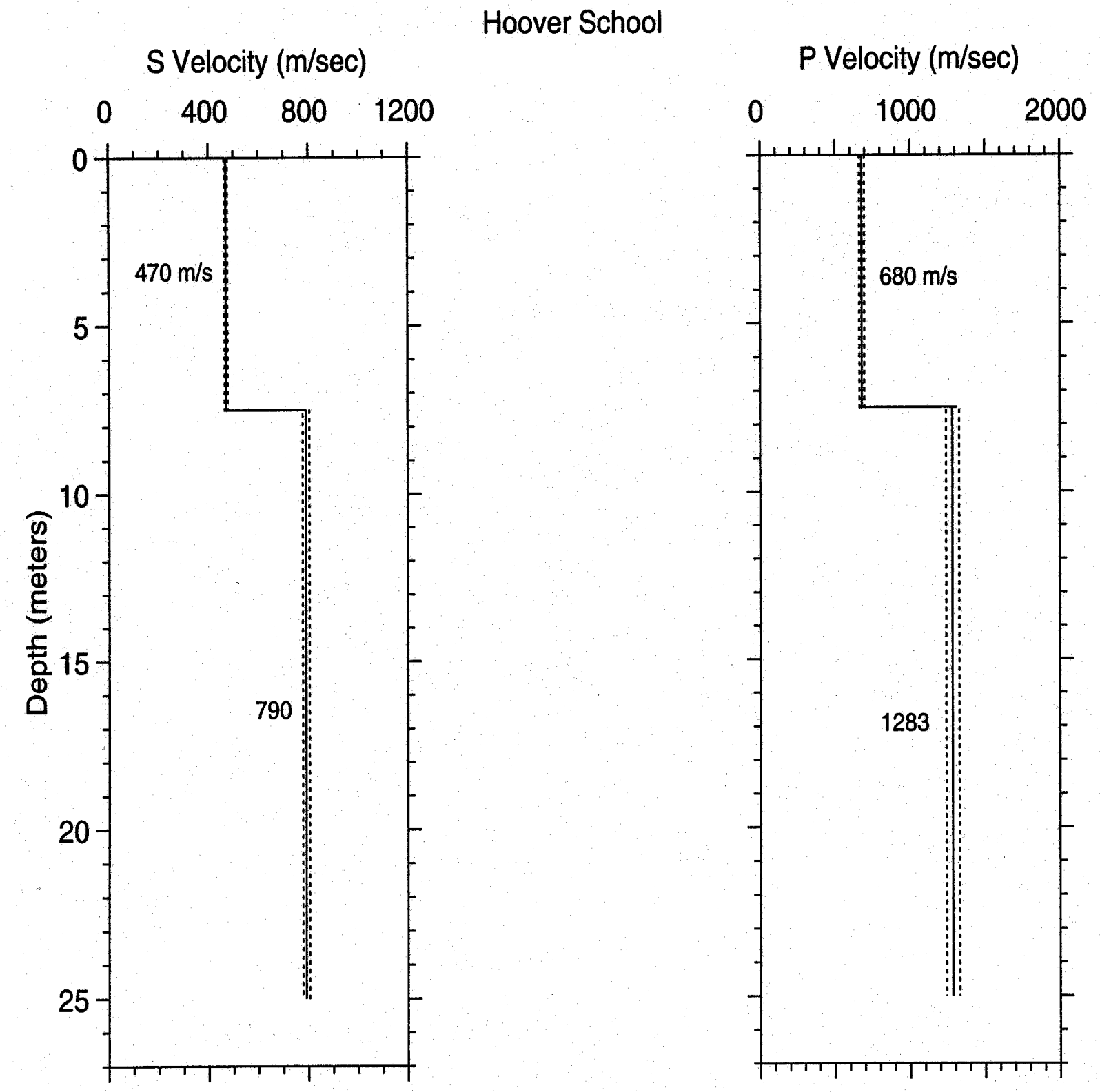

Figure A-25. S- and P-wave velocity profiles with dashed lines representing one standard deviation. Lithology is not available from this borehole. 
ABLE $\$$-9. S-wave arrival times and velocity sumaries.

Location: Hoover School: S hoffset $=3.00$ travel-time file: $\mathrm{P}: \backslash$ HOO $\backslash$ HOOSZ TT

$\begin{array}{rrrcccr}d(\mathbb{I}) & d(f t) & t s 1(s) & \text { tvrt (s) } & \text { vavg (in/s) } & \text { sig } & \text { rsdl (sec) } \\ 2.5 & 8.2 & 0.0087 & 0.0053 & 470 & 1 & 0.0004 \\ 5.0 & 16.4 & 0.0122 & 0.0106 & 470 & 1 & -0.0002 \\ 7.5 & 24.6 & 0.0166 & 0.0160 & 470 & 1 & -0.0006 \\ 10.0 & 32.8 & 0.0200 & 0.0191 & 523 & 1 & 0.0001 \\ 12.5 & 41.0 & 0.0232 & 0.0223 & 561 & 1 & 0.0003 \\ 15.0 & 49.2 & 0.0262 & 0.0255 & 589 & 1 & 0.0003 \\ 17.5 & 57.4 & 0.0291 & 0.0286 & 612 & 1 & 0.0001 \\ 20.0 & 65.6 & 0.0323 & 0.0318 & 629 & 1 & 0.0002 \\ 22.5 & 73.8 & 0.0355 & 0.0349 & 644 & 1 & 0.0003 \\ 25.0 & 82.0 & 0.0377 & 0.0381 & 656 & 1 & -0.0007\end{array}$

$33.98491-118.02890$ Hole_Code: 299 nlayers $=2$

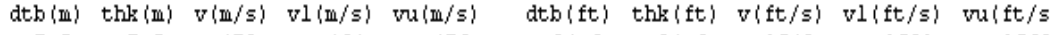
$\begin{array}{llllllllll}7.5 & 7.5 & 470 & 464 & 476 & 24.6 & 24.6 & 1542 & 1521 & 1563\end{array}$

$\begin{array}{rrrrrrrrrr}7.5 & 7.5 & 470 & 464 & 476 & 24.6 & 24.6 & 1542 & 1521 & 1563 \\ 25.0 & 17.5 & 790 & 777 & 804 & 82.0 & 57.4 & 2593 & 2549 & 2639\end{array}$

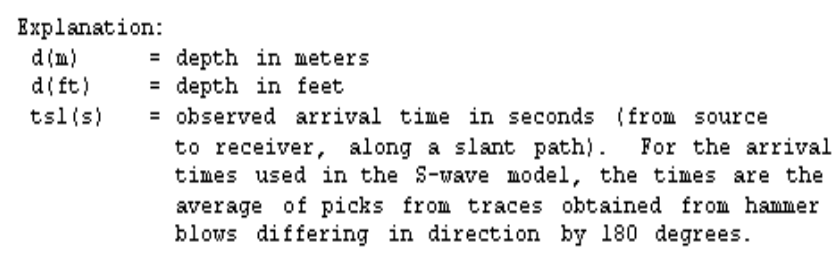


ABLE A-10. P-wave arrival times and velocity summaries.

Location: Hoover School: p Coordinates: hoffset $=3.00$ travel-time file: $\mathrm{P}: \backslash \mathrm{HOO} \backslash \mathrm{H} 0 \mathrm{OP} 2 . \mathrm{TT}$

$33.98491-118.02890$ Hole_Code: 299 nlayers $=2$

$\begin{array}{rrrrrrr}d(\mathrm{~m}) & \mathrm{d}(\mathrm{ft}) & \mathrm{t} s l(s) & \text { tvrt }(s) & \text { vavg }(\mathrm{m} / \mathrm{s}) & \text { sig } & \mathrm{rsdl}(\mathrm{sec}) \\ 2.5 & 8.2 & 0.0066 & 0.0037 & 680 & 1 & 0.0009 \\ 5.0 & 16.4 & 0.0090 & 0.0074 & 680 & 1 & 0.0004 \\ 7.5 & 24.6 & 0.0114 & 0.0110 & 680 & 1 & -0.0005 \\ 10.0 & 32.8 & 0.0129 & 0.0130 & 771 & 1 & -0.0006 \\ 12.5 & 41.0 & 0.0153 & 0.0149 & 837 & 1 & 0.0000 \\ 15.0 & 49.2 & 0.0177 & 0.0169 & 889 & 1 & 0.0005 \\ 17.5 & 57.4 & 0.0192 & 0.0188 & 930 & 1 & 0.0001 \\ 20.0 & 65.6 & 0.0207 & 0.0208 & 963 & 1 & -0.0003 \\ 22.5 & 73.8 & 0.0228 & 0.0227 & 990 & 1 & -0.0001 \\ 25.0 & 82.0 & 0.0249 & 0.0247 & 1013 & 1 & 0.0001\end{array}$

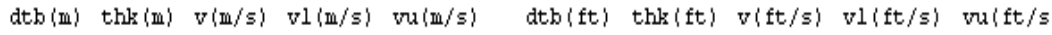

$\begin{array}{rrrrrrrrrr}7.5 & 7.5 & 680 & 664 & 697 & 24.6 & 24.6 & 2231 & 2179 & 2286 \\ 25.0 & 17.5 & 1283 & 1241 & 1328 & 82.0 & 57.4 & 4211 & 4072 & 4359\end{array}$

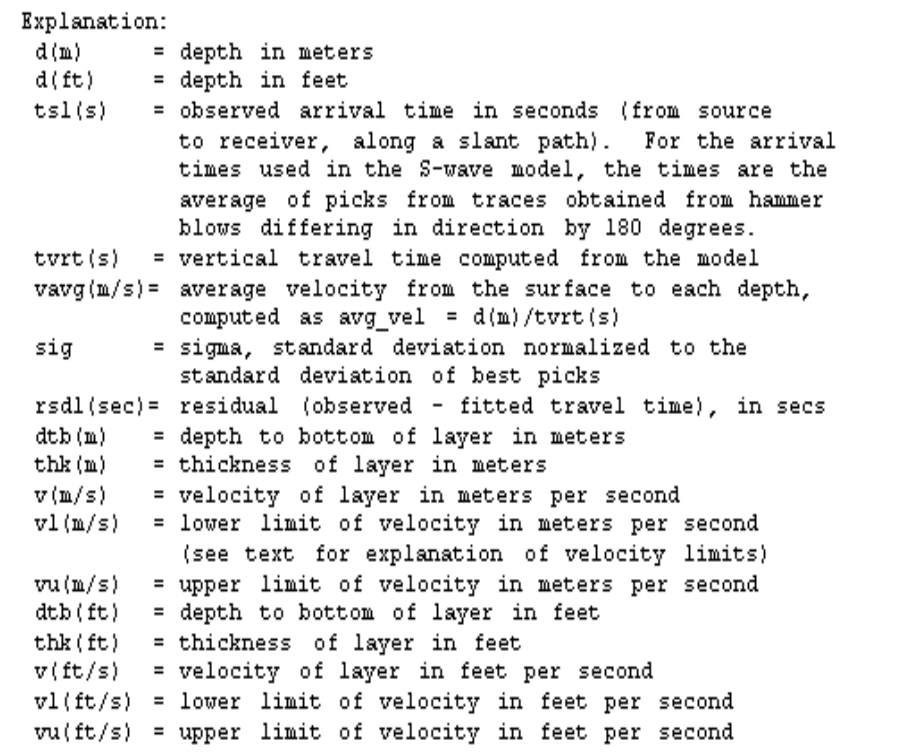




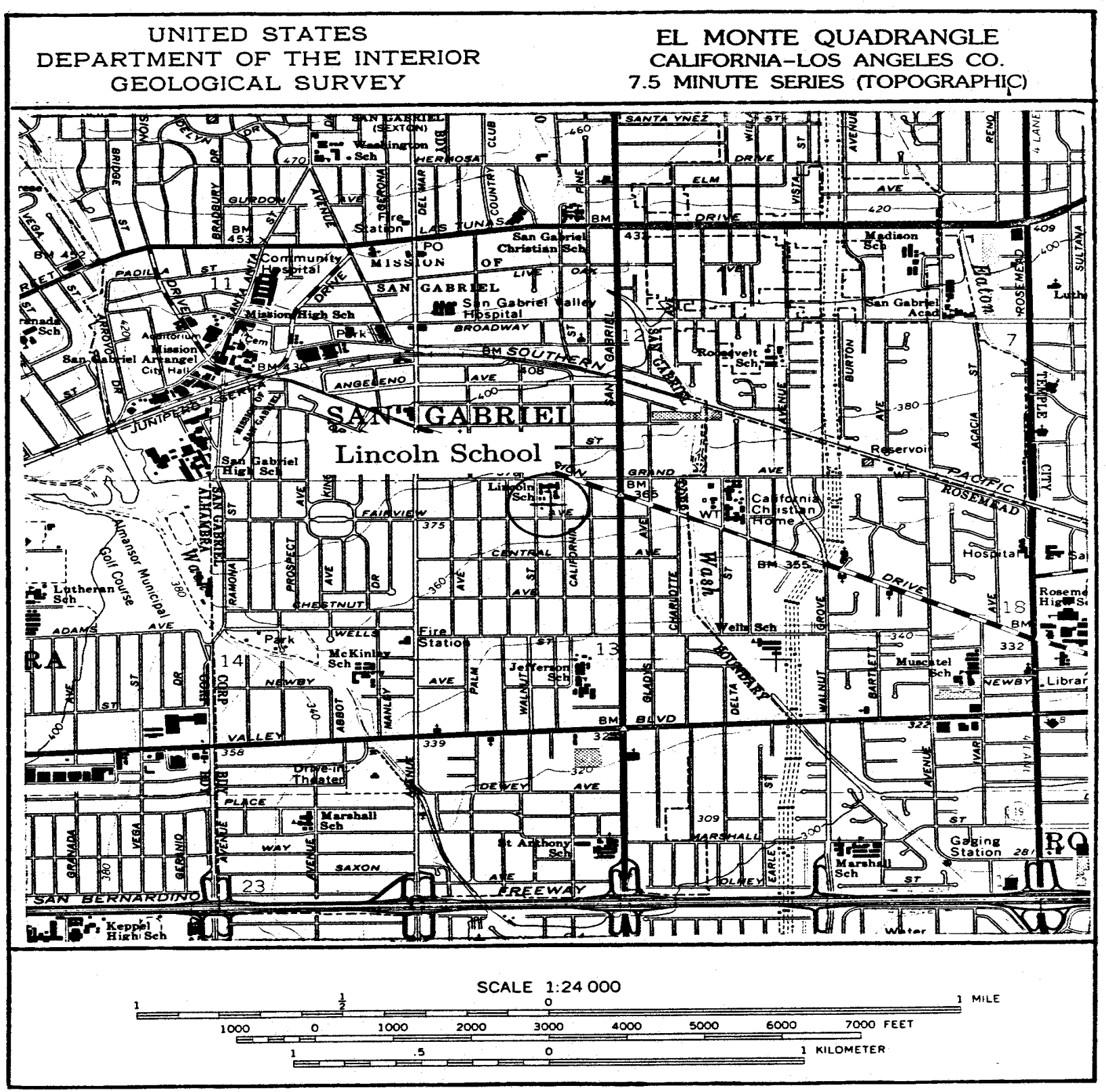

Figure A-26. Site location map for the borehole at Lincoln School. The accelerograph is located approximately 91 meters from the borehole. 


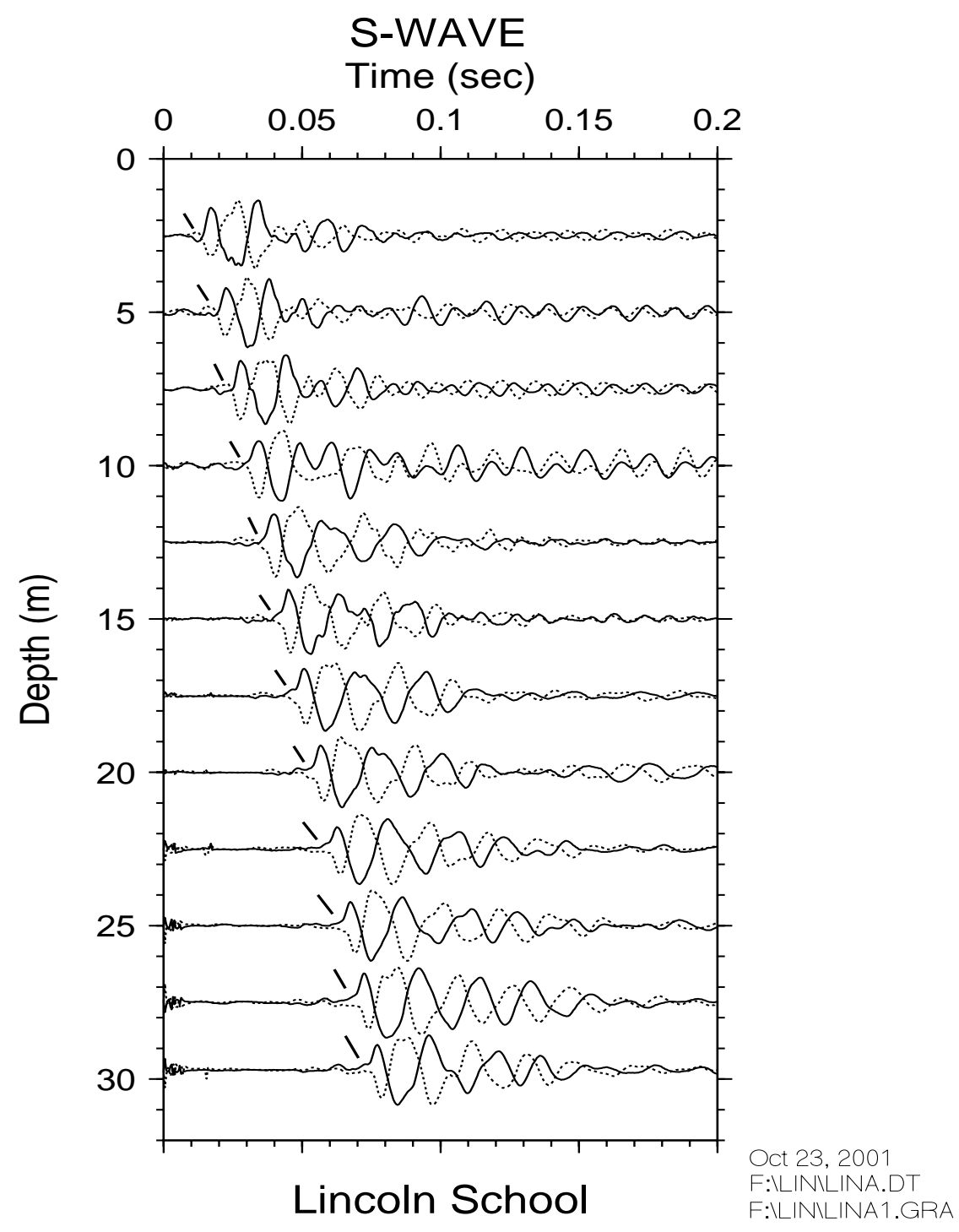

Figure A-27. Horizontal component record section (from impacts in opposite directions) superimposed for identification of S-wave onset. Approximate S-wave time picks are indicated by the hatch marks. 


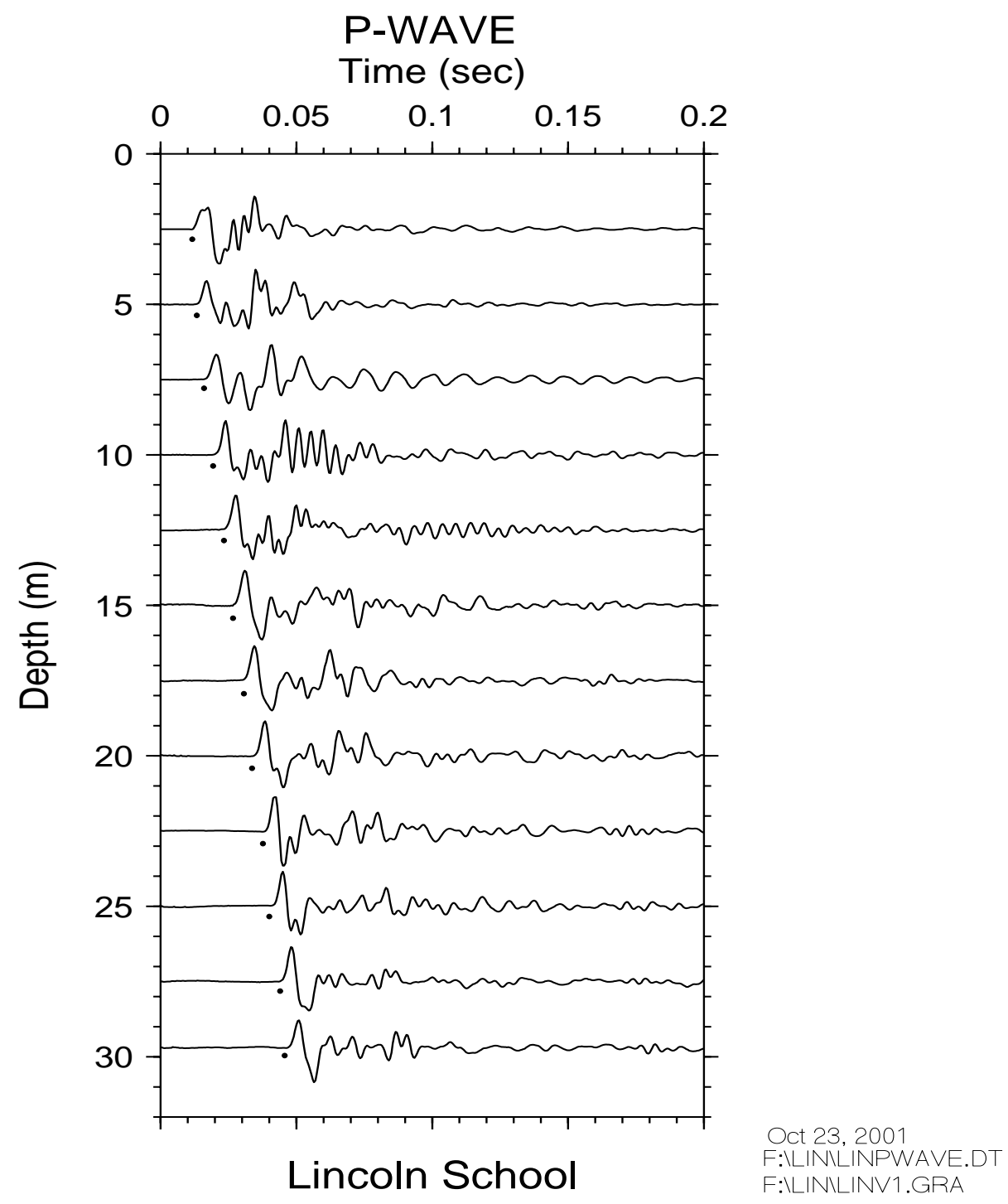

Figure A-28. Vertical component record section. Approximate P-wave arrivals are indicated by the dots. 


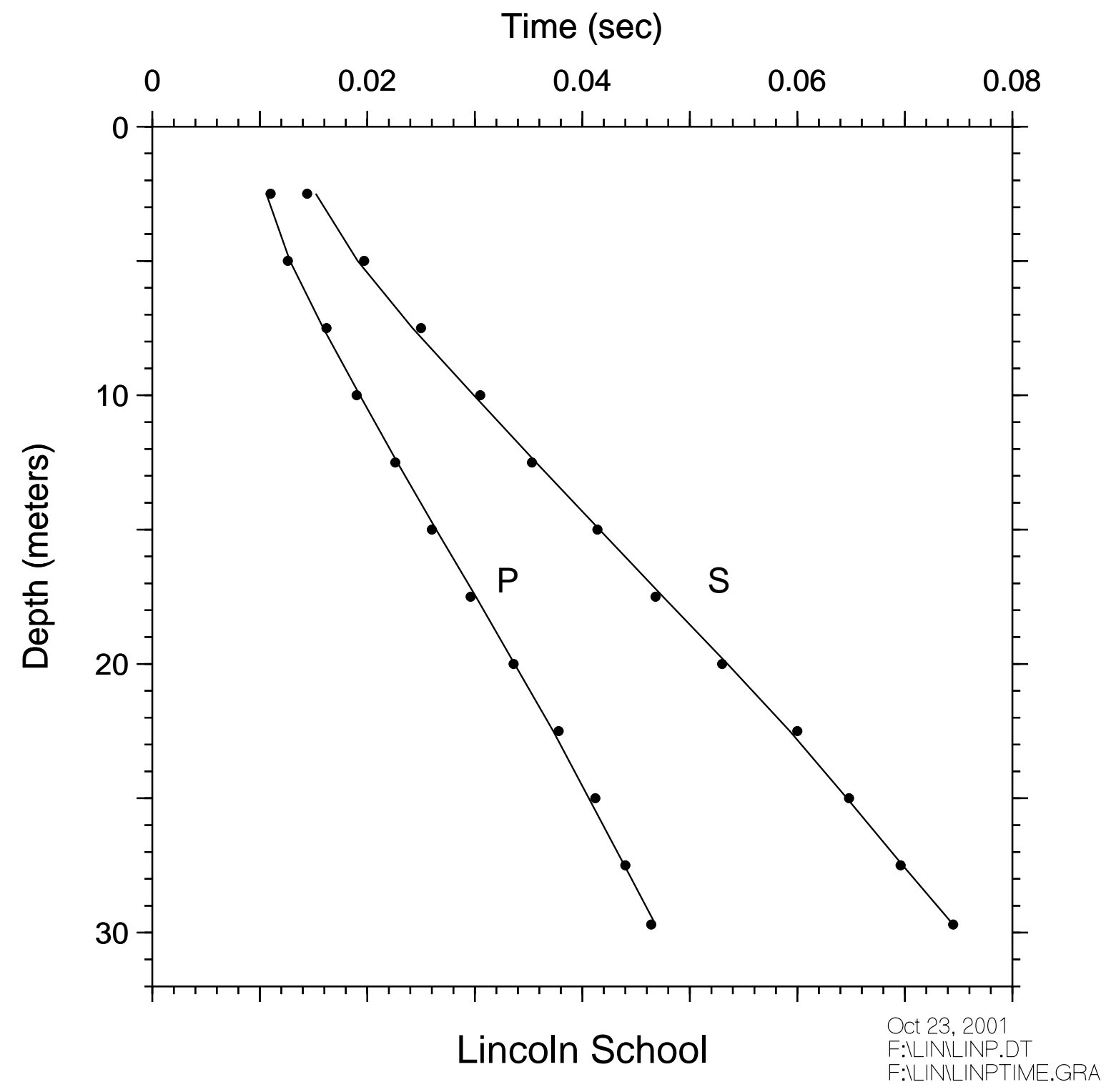

Figure A-29. Time-depth graph of P-wave and S-wave picks. Line segments are straightline interpolations of model predictions at the observation depths. The times for zero depth, not shown, are given by hoffset divided by the velocity in the uppermost layer (see accompanying tables of velocities for specific values). 


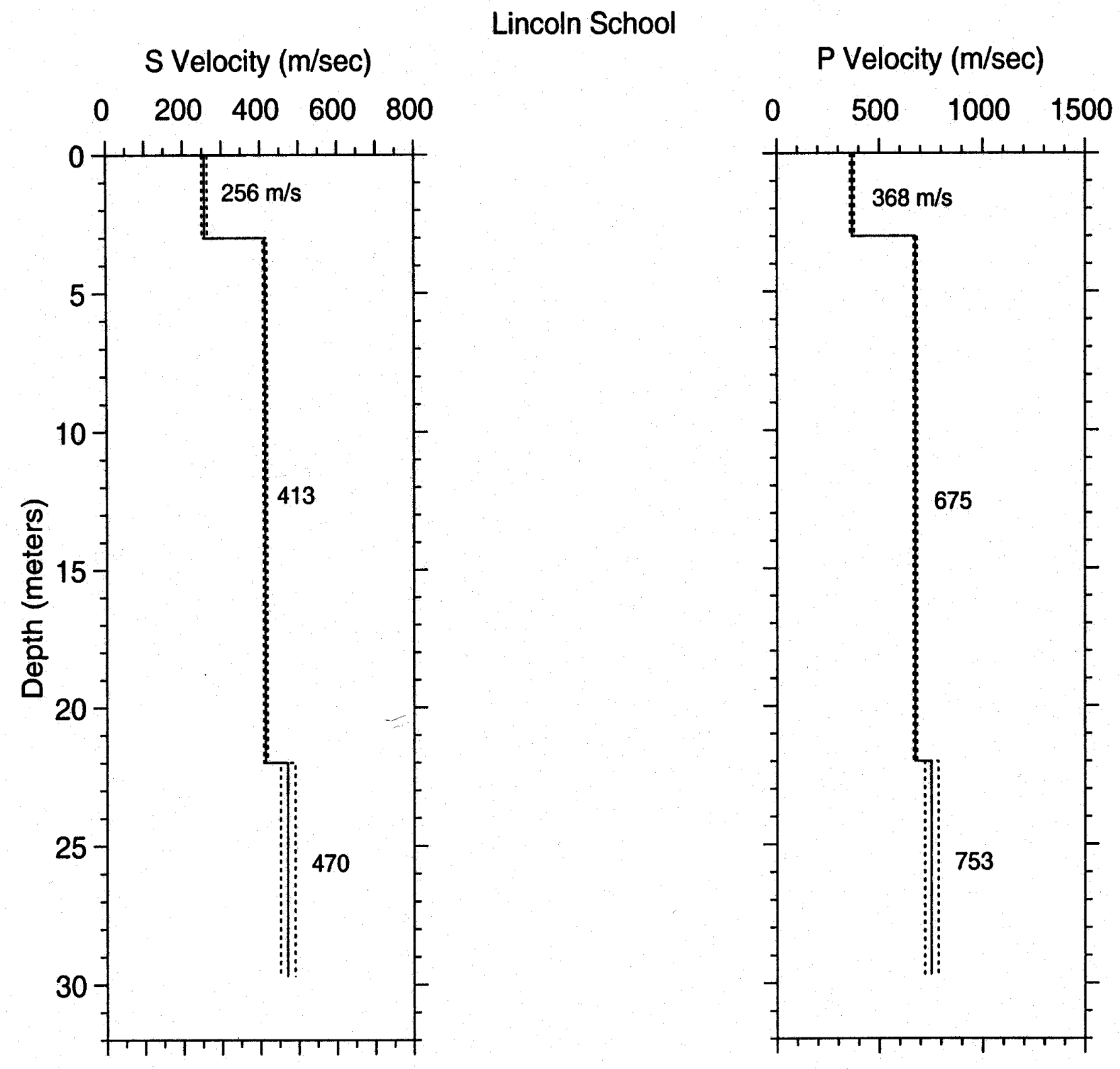

Figure A-30. S- and P-wave velocity profiles with dashed lines representing one standard deviation. Lithology is not available from this borehole. 
ABLE A-11. S-wave arrival times and velocity summaries. Location: Lincoln School: $\$$
hoffset $=3.00$ travel-time file: $\mathrm{P}: \backslash$ LIN $\backslash$ LINS2.TT

$\begin{array}{rrrrccc}d(\mathrm{~m}) & \mathrm{d}(\mathrm{ft}) & \mathrm{t} s 1(s) & \text { tvrt }(s) & \text { vavg }(\mathrm{m} / \mathrm{s}) & \text { sig } & \mathrm{rsdl}(\mathrm{sec}) \\ 2.5 & 8.2 & 0.0144 & 0.0098 & 256 & 1 & -0.0008 \\ 5.0 & 16.4 & 0.0197 & 0.0166 & 302 & 1 & 0.0006 \\ 7.5 & 24.6 & 0.0250 & 0.0226 & 332 & 1 & 0.0008 \\ 10.0 & 32.8 & 0.0305 & 0.0287 & 349 & 1 & 0.0006 \\ 12.5 & 41.0 & 0.0353 & 0.0347 & 360 & 1 & -0.0004 \\ 15.0 & 49.2 & 0.0414 & 0.0408 & 368 & 1 & -0.0002 \\ 17.5 & 57.4 & 0.0468 & 0.0468 & 374 & 1 & -0.0007 \\ 20.0 & 65.6 & 0.0530 & 0.0529 & 378 & 1 & -0.0005 \\ 22.5 & 73.8 & 0.0600 & 0.0588 & 383 & 1 & 0.0007 \\ 25.0 & 82.0 & 0.0648 & 0.0641 & 390 & 1 & 0.0002 \\ 27.5 & 90.2 & 0.0696 & 0.0694 & 396 & 1 & -0.0002 \\ 29.7 & 97.4 & 0.0745 & 0.0741 & 401 & 1 & 0.0000\end{array}$

$34.09043-118.09300$ Hole_Code: 300 nlayers $=3$

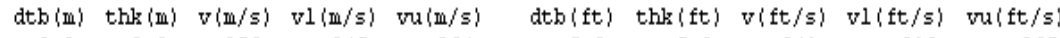

$\begin{array}{rrrrrrrrrr}3.0 & 3.0 & 256 & 249 & 264 & 9.8 & 9.8 & 841 & 818 & 865 \\ 22.0 & 19.0 & 413 & 408 & 418 & 72.2 & 62.3 & 1354 & 1338 & 1371 \\ 29.7 & 7.7 & 470 & 452 & 490 & 97.4 & 25.3 & 1544 & 1484 & 1609\end{array}$

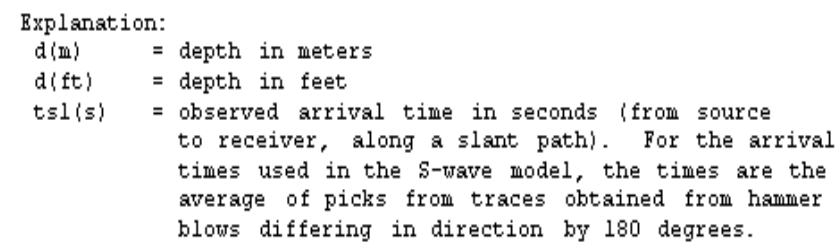


ABLE A-12. P-wave arrival times and velocity summaries.

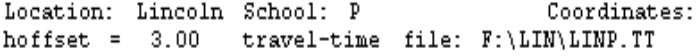

$\begin{array}{rrrcccr}d(\mathbb{I}) & d(f t) & t s l(s) & t v r t(s) & v a v g(\mathbb{m} / s) & s i g & r s d l(s e c) \\ 2.5 & 8.2 & 0.0110 & 0.0068 & 368 & 1 & 0.0004 \\ 5.0 & 16.4 & 0.0126 & 0.0111 & 450 & 1 & -0.0002 \\ 7.5 & 24.6 & 0.0162 & 0.0148 & 506 & 1 & 0.0003 \\ 10.0 & 32.8 & 0.0190 & 0.0185 & 540 & 1 & -0.0003 \\ 12.5 & 41.0 & 0.0226 & 0.0222 & 562 & 1 & -0.0002 \\ 15.0 & 49.2 & 0.0260 & 0.0259 & 578 & 1 & -0.0004 \\ 17.5 & 57.4 & 0.0296 & 0.0296 & 591 & 1 & -0.0004 \\ 20.0 & 65.6 & 0.0336 & 0.0333 & 600 & 1 & -0.0001 \\ 22.5 & 73.8 & 0.0378 & 0.0370 & 609 & 1 & 0.0005 \\ 25.0 & 82.0 & 0.0412 & 0.0403 & 621 & 1 & 0.0006 \\ 27.5 & 90.2 & 0.0440 & 0.0436 & 631 & 1 & 0.0001 \\ 29.7 & 97.4 & 0.0464 & 0.0465 & 638 & 1 & -0.0003\end{array}$

$34.09044-118.09306$ Hole_Code: 300 nlayers $=3$

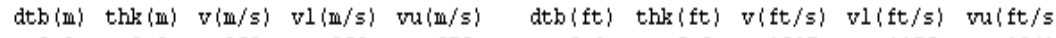
$\begin{array}{rrrrrrrrrr}3.0 & 3.0 & 368 & 358 & 378 & 9.8 & 9.8 & 1207 & 1175 & 1241 \\ 22.0 & 19.0 & 675 & 666 & 684 & 72.2 & 62.3 & 2213 & 2184 & 2243 \\ 29.7 & 7.7 & 753 & 722 & 787 & 97.4 & 25.3 & 2470 & 2367 & 2582\end{array}$

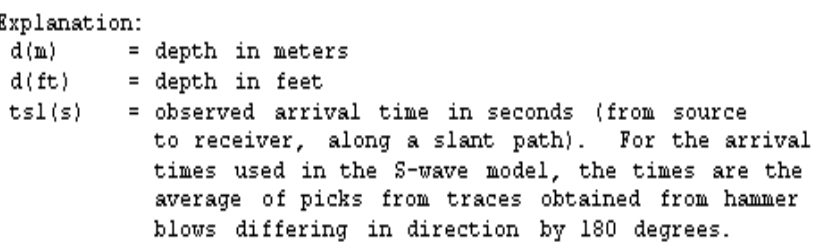




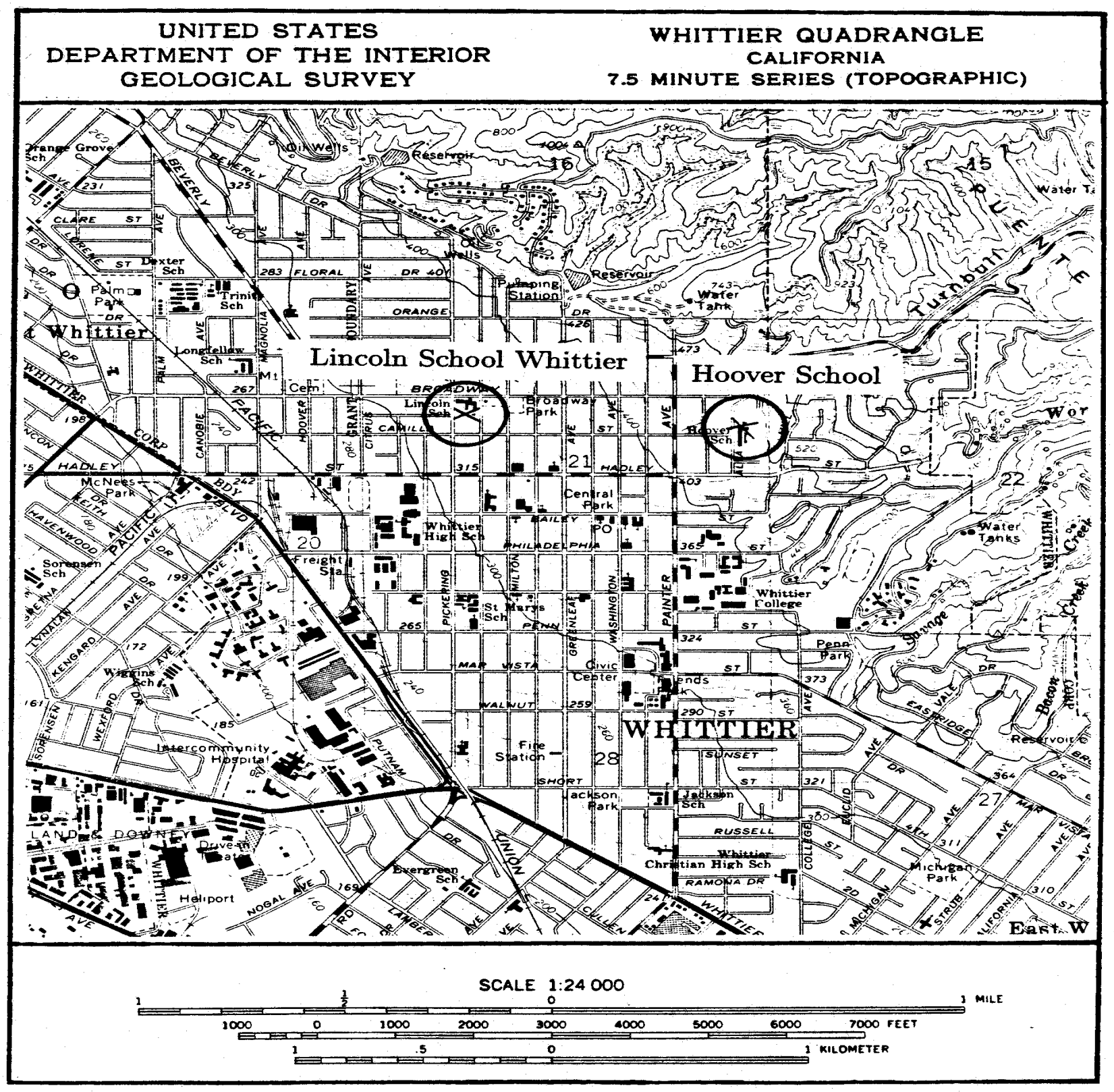

Figure A-31. Site location map for the borehole at Lincoln School Whittier. 


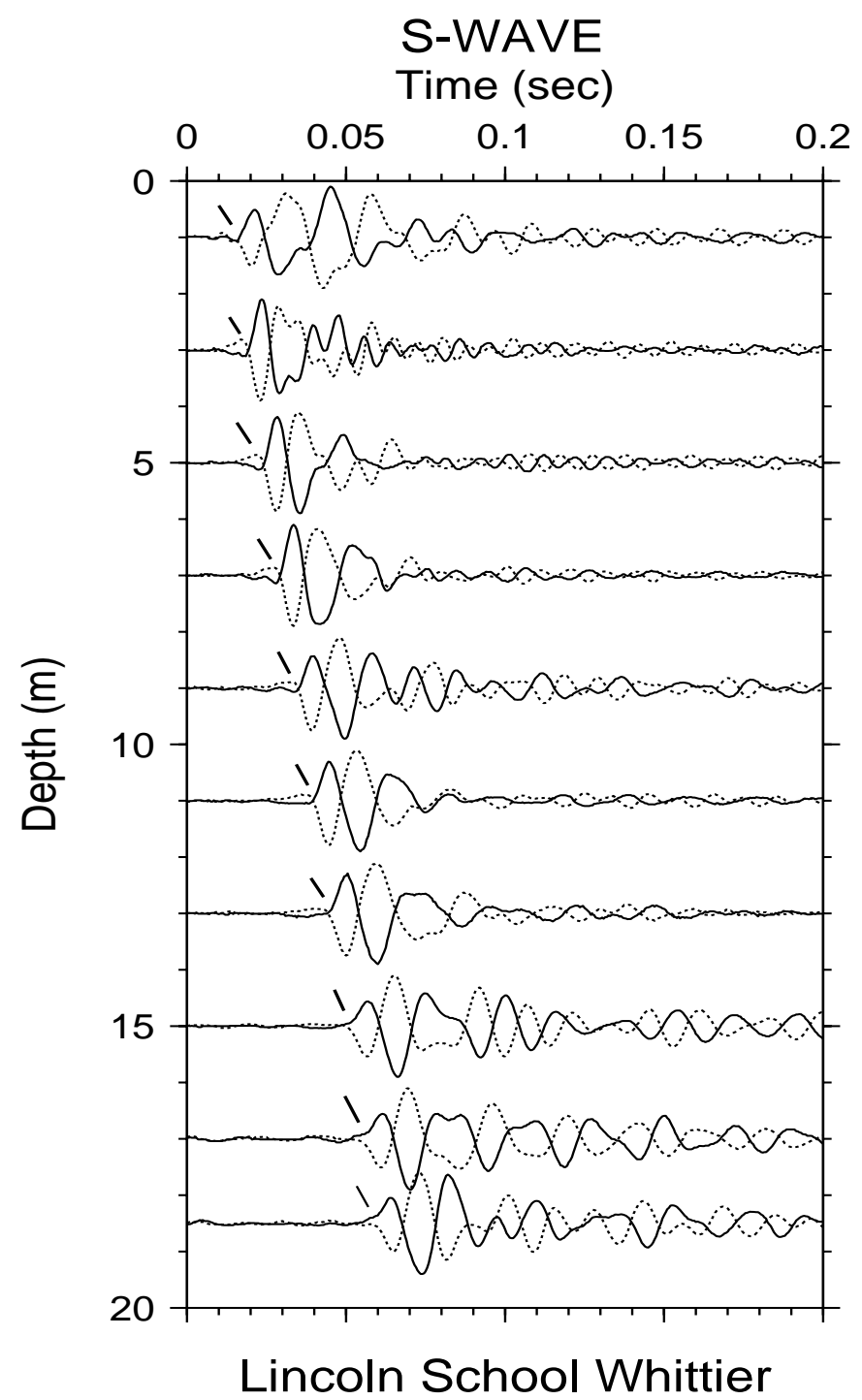

Figure A-32. Horizontal component record section (from impacts in opposite directions) superimposed for identification of S-wave onset. Approximate S-wave time picks are indicated by the hatch marks. 


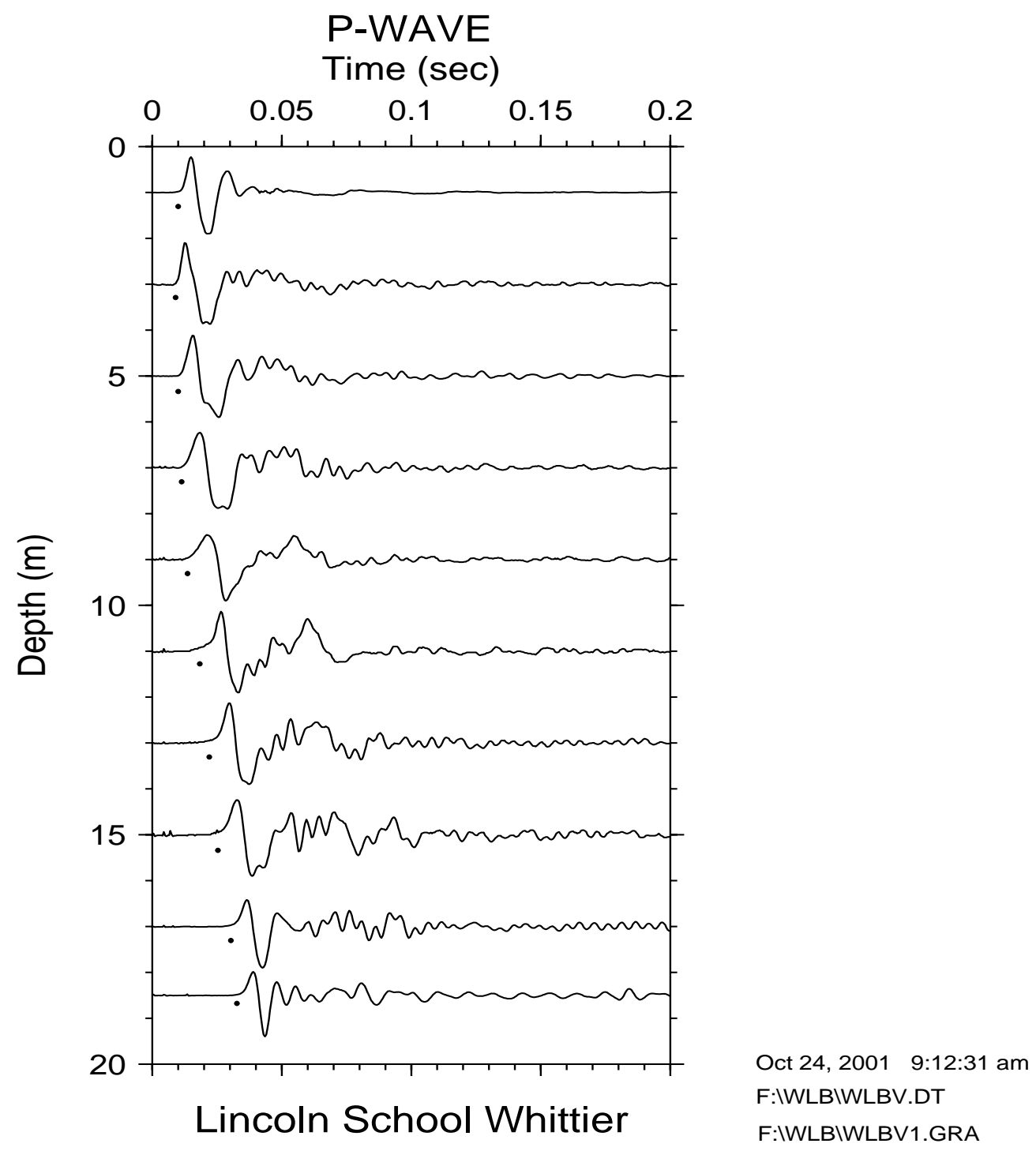

Figure A-33. Vertical component record section. Approximate P-wave arrivals are indicated by the dots. 


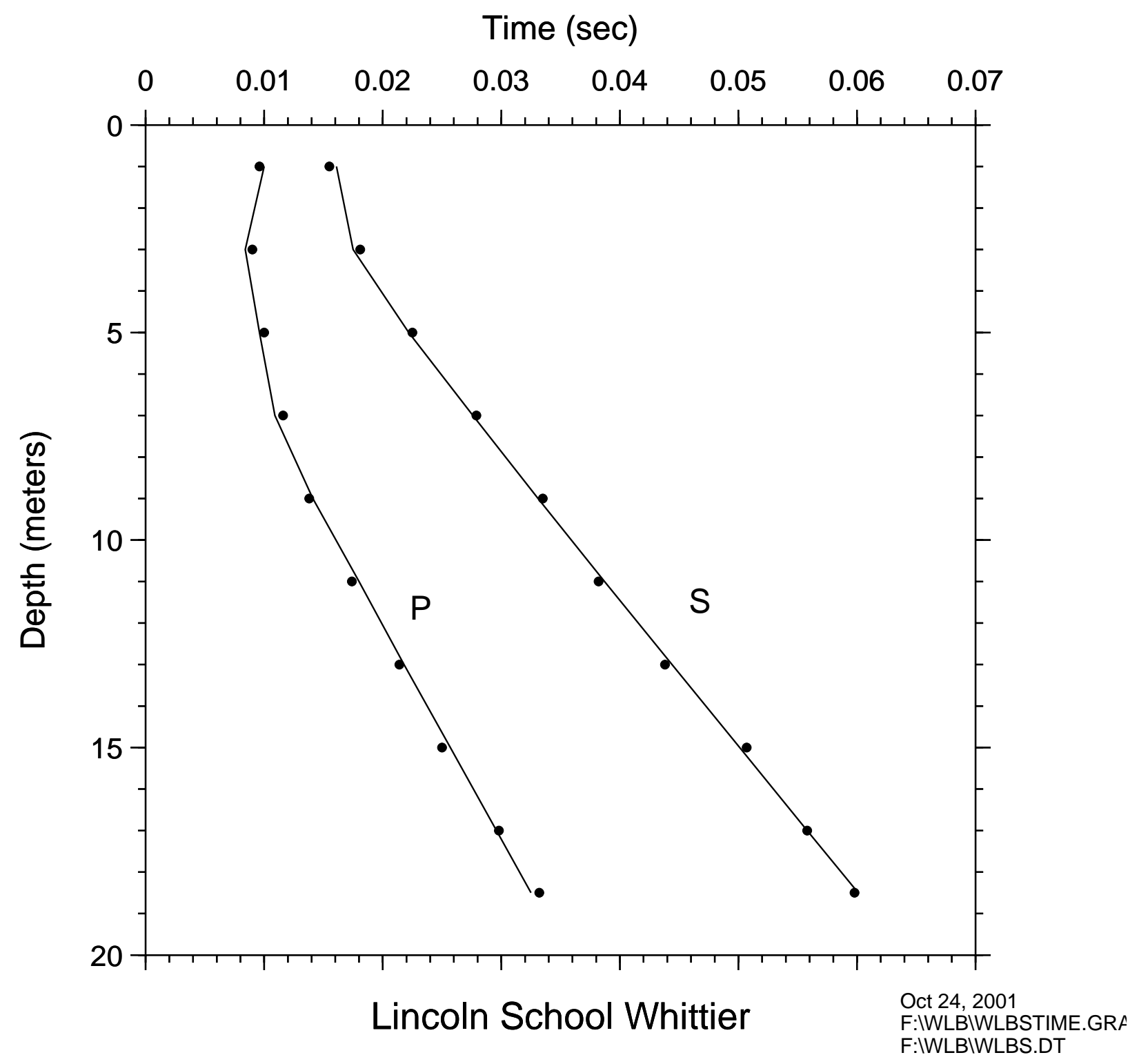

Figure A-34. Time-depth graph of P-wave and S-wave picks. Line segments are straightline interpolations of model predictions at the observation depths. The times for zero depth, not shown, are given by hoffset divided by the velocity in the uppermost layer (see accompanying tables of velocities for specific values). 


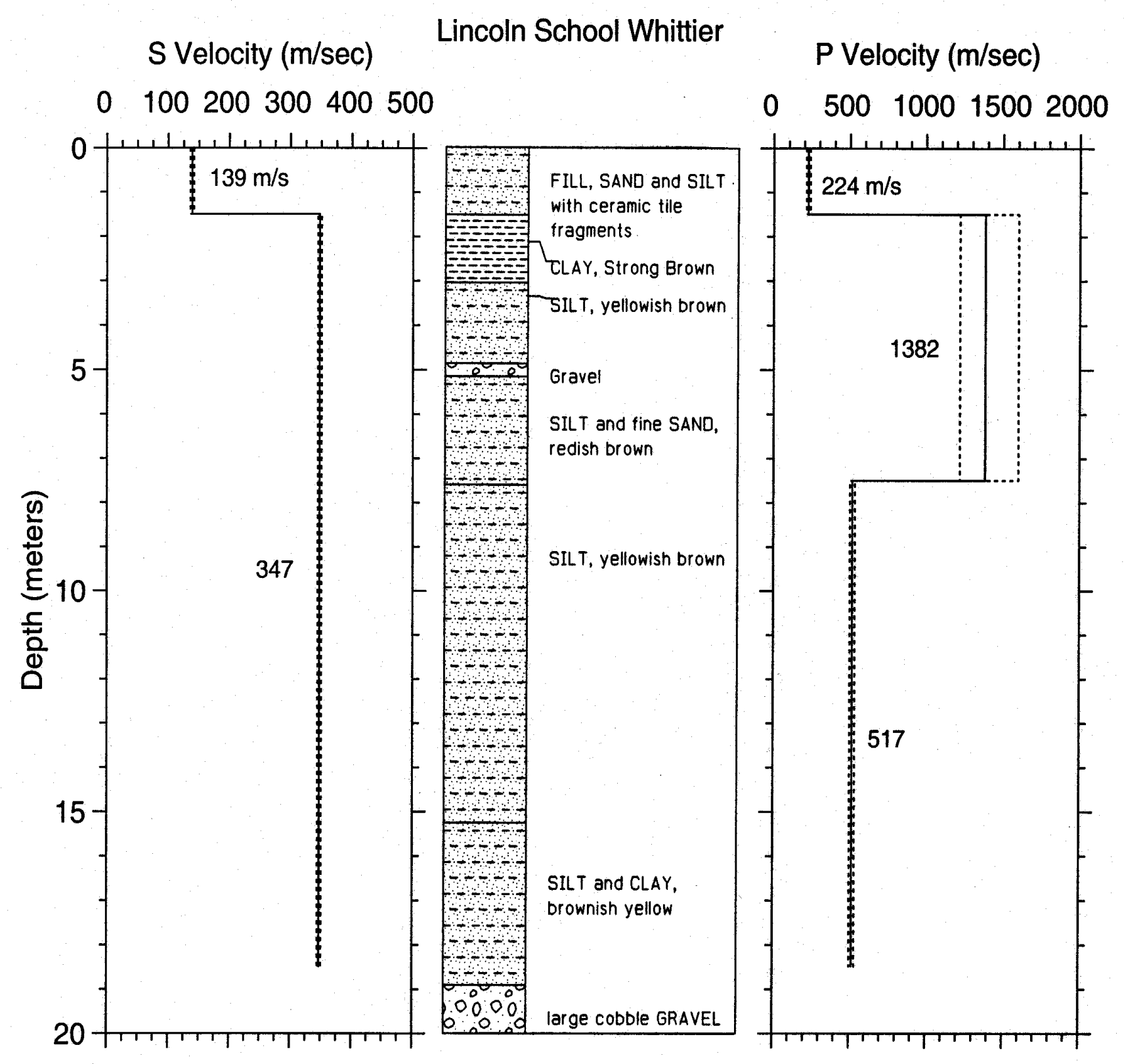

Figure A-35. S- and P-wave velocity profiles. Dashed lines represent one standard deviation. Lithology is shown for correlation with velocities. 
ABLE A-13. S-wave arrival times and velocity sumaries.

Location: Lincoln School - Thittier: \& Coordinates: hoffset $=2.00$ travel-time file: $\mathbb{P}: \backslash$ WLB $\backslash$ WLBS. Tr

$\begin{array}{rrrrrrr}d(m) & d(f t) & t s l(s) & t v r t(s) & v a v g(m / s) & s i g & r s d l(s e c) \\ 1.0 & 3.3 & 0.0155 & 0.0072 & 139 & 1 & -0.0006 \\ 3.0 & 9.8 & 0.0181 & 0.0151 & 198 & 1 & 0.0006 \\ 5.0 & 16.4 & 0.0225 & 0.0209 & 239 & 1 & 0.0003 \\ 7.0 & 23.0 & 0.0279 & 0.0266 & 263 & 1 & 0.0003 \\ 9.0 & 29.5 & 0.0335 & 0.0324 & 278 & 1 & 0.0004 \\ 11.0 & 36.1 & 0.0382 & 0.0382 & 288 & 1 & -0.0005 \\ 13.0 & 42.7 & 0.0438 & 0.0439 & 296 & 1 & -0.0006 \\ 15.0 & 49.2 & 0.0507 & 0.0497 & 302 & 1 & 0.0007 \\ 17.0 & 55.8 & 0.0558 & 0.0555 & 307 & 1 & 0.0000 \\ 18.5 & 60.7 & 0.0598 & 0.0598 & 309 & 1 & -0.0002\end{array}$

$33.98535-118.04060$ Hole_Code: 301 nlayers $=2$

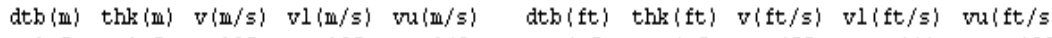

$\begin{array}{rrrrrrrrrr}1.5 & 1.5 & 139 & 135 & 142 & 4.9 & 4.9 & 455 & 444 & 466 \\ 18.5 & 17.0 & 347 & 344 & 351 & 60.7 & 55.8 & 1140 & 1130 & 1150\end{array}$

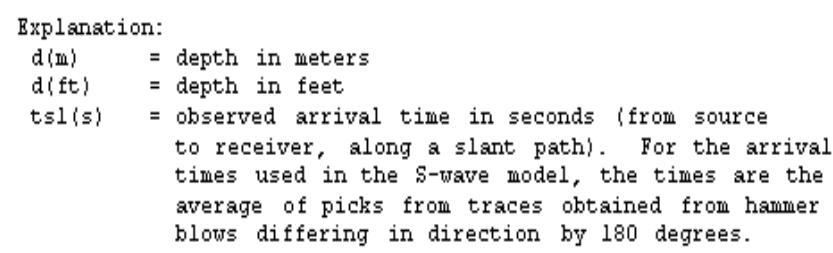


ABLE A-14. S-wave arrival times and velocity sumaries. $\begin{array}{ll}\text { Location: Lincoln School thittier: } \mathrm{p} & \text { Coordinates: } \\ \text { hoffset }=2.00 & \text { travel-time file: } \mathrm{P}: \backslash \text { WLB } \backslash \text { WLBVBRT. TT }\end{array}$

$\begin{array}{rrrrcrr}d(m) & d(f t) & t s l(s) & t v r t(s) & v a v g(m / s) & s i g & r s d l(s e c) \\ 1.0 & 3.3 & 0.0096 & 0.0045 & 224 & 1 & -0.0004 \\ 3.0 & 9.8 & 0.0090 & 0.0078 & 386 & 1 & 0.0006 \\ 5.0 & 16.4 & 0.0100 & 0.0092 & 542 & 1 & 0.0004 \\ 7.0 & 23.0 & 0.0116 & 0.0107 & 656 & 1 & 0.0007 \\ 9.0 & 29.5 & 0.0138 & 0.0139 & 646 & 1 & -0.0003 \\ 11.0 & 36.1 & 0.0174 & 0.0178 & 618 & 1 & -0.0006 \\ 13.0 & 42.7 & 0.0214 & 0.0217 & 600 & 1 & -0.0004 \\ 15.0 & 49.2 & 0.0250 & 0.0255 & 587 & 1 & -0.0007 \\ 17.0 & 55.8 & 0.0298 & 0.0294 & 578 & 1 & 0.0002 \\ 18.5 & 60.7 & 0.0332 & 0.0323 & 572 & 1 & 0.0007\end{array}$

$33.98535-118.04060$ Hole_Code: 301

nlayers $=3$

dth (II) thk(II) $\mathrm{v}(\mathrm{II} / \mathrm{s}) \mathrm{vl}(\mathrm{II} / \mathrm{s}) \quad \mathrm{vu}(\mathrm{II} / \mathrm{s}$

$\begin{array}{rrrrr}1.5 & 1.5 & 224 & 212 & 238\end{array}$

$11.0-1317-1217-15901-533$

dtb $(\mathrm{ft}) \quad \operatorname{thl}(\mathrm{ft}) \quad \mathrm{V}(\mathrm{ft} / \mathrm{s}) \quad \mathrm{vl}(\mathrm{ft} / \mathrm{s}) \quad \mathrm{vu}(\mathrm{ft} / \mathrm{s})$ $\begin{array}{rrrrr}4.9 & 4.9 & 736 & 695 & 782 \\ 24.6 & 19.7 & 4535 & 3994 & 5825\end{array}$

$\begin{array}{lllll}24.6 & 19.7 & 4535 & 3994 & 5245 \\ 60.7 & 36.1 & 1695 & 1644 & 1749\end{array}$

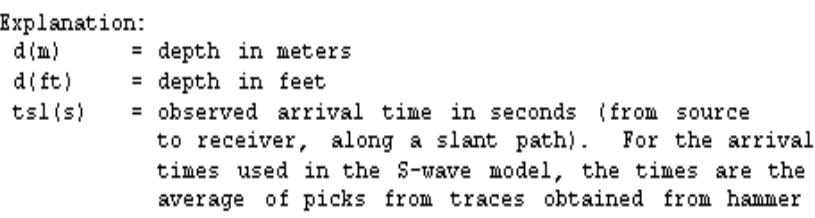






Figure A-36. Site location map for the borehole at Los Alisos Adult School. 


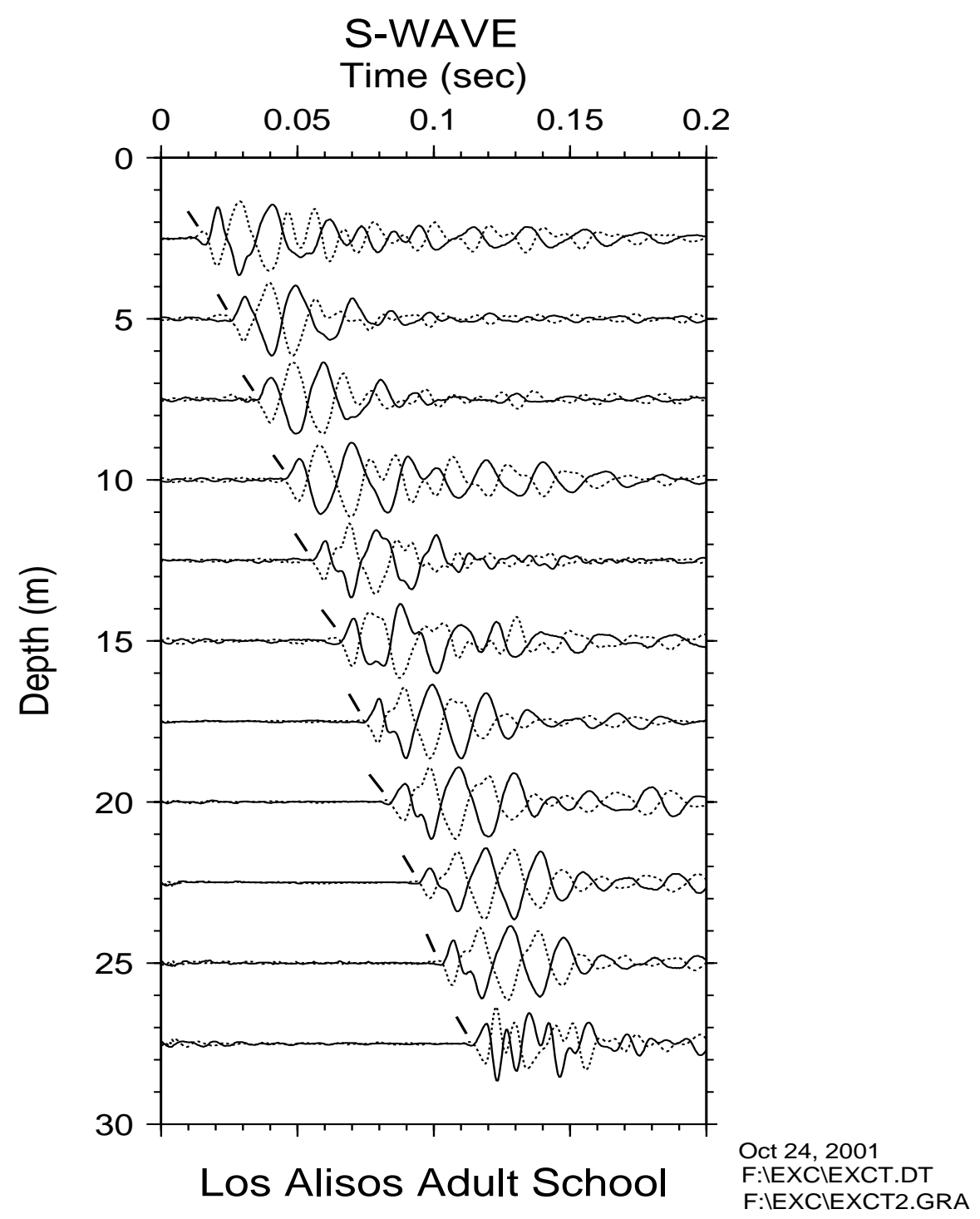

Figure A-37. Horizontal component record section (from impacts in opposite directions) superimposed for identification of S-wave onset. Approximate S-wave time picks are indicated by the hatch marks. 


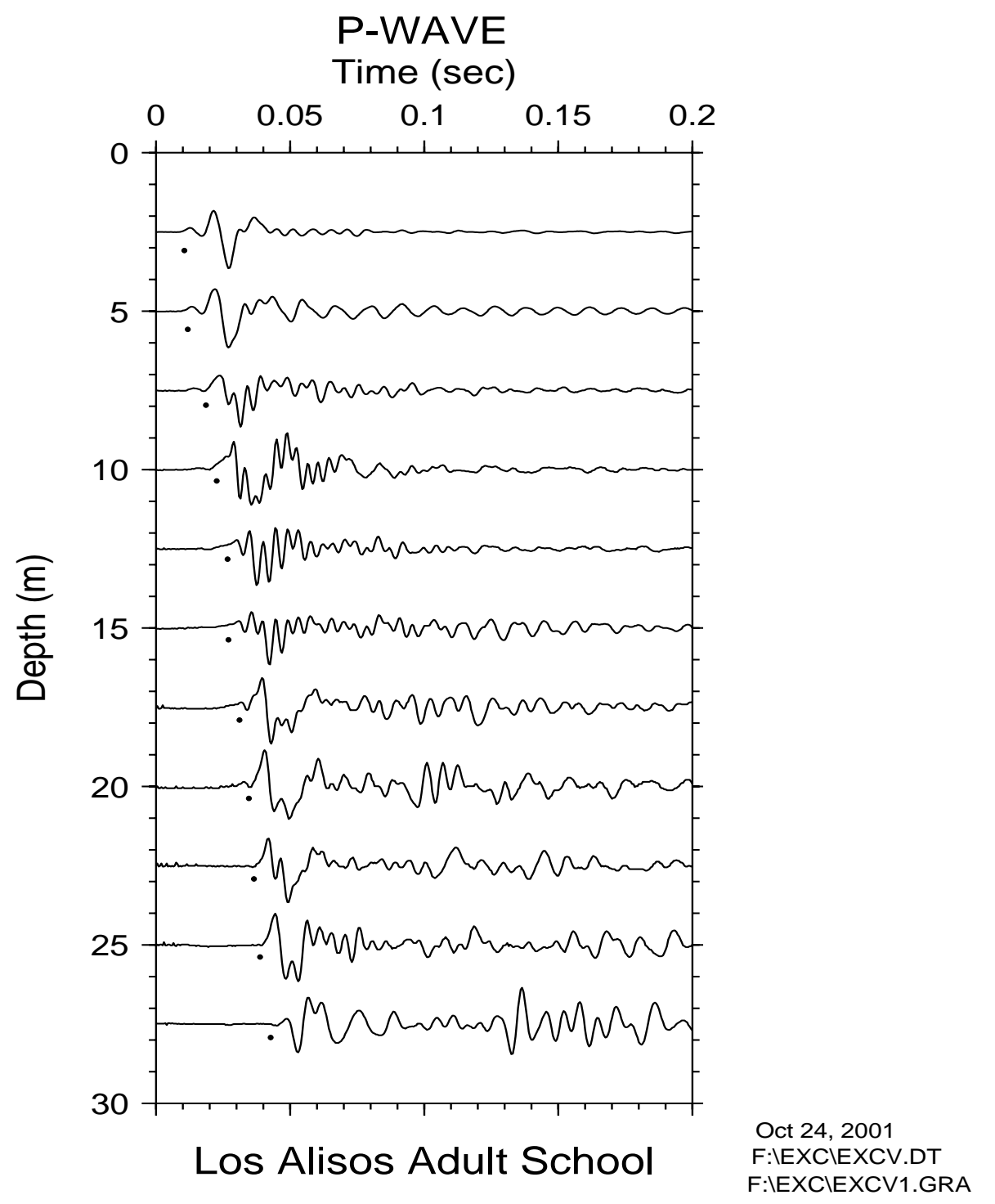

Figure A-38. Vertical component record section. Approximate P-wave arrivals are indicated by the dots. 


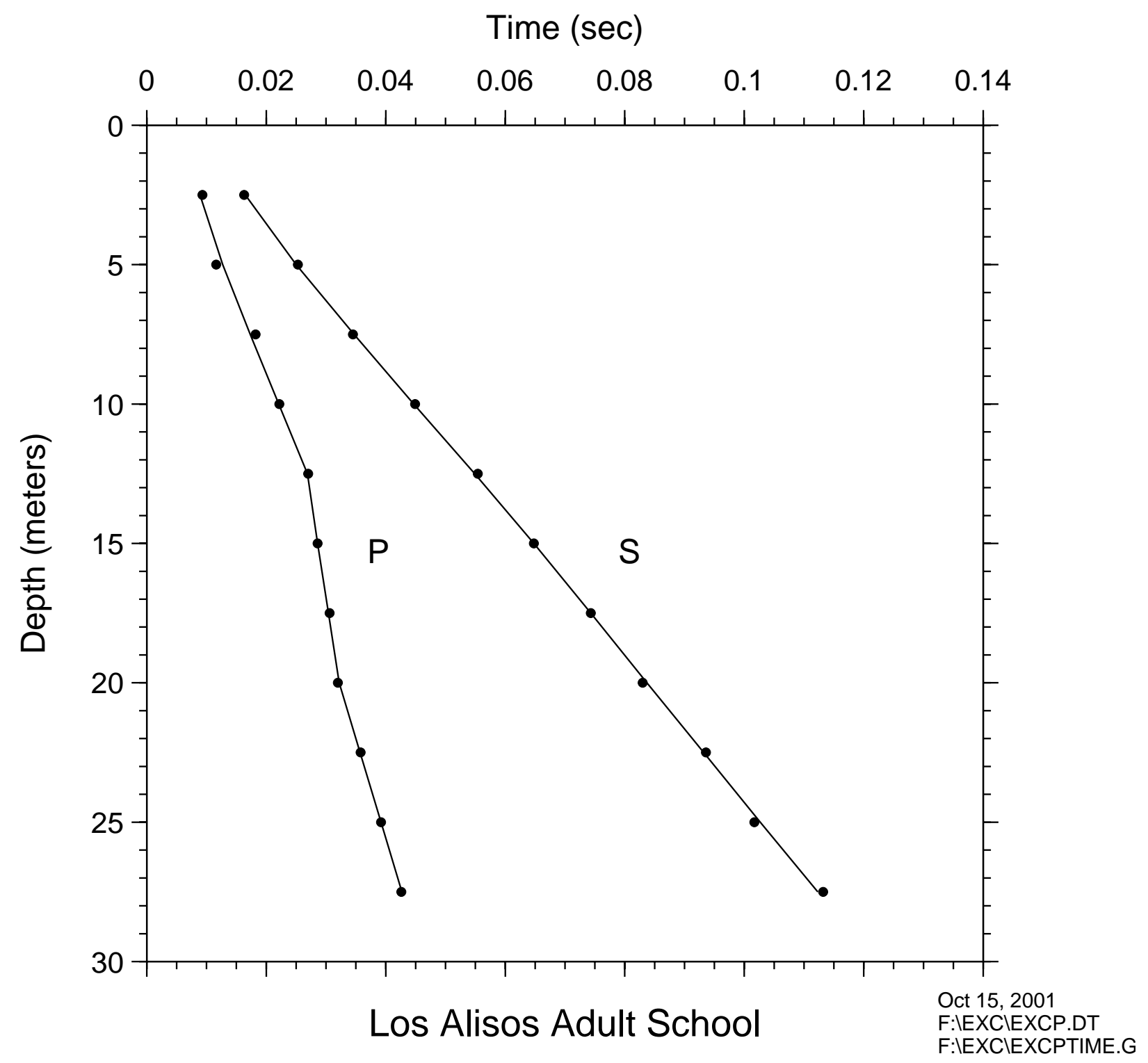

Figure A-39. Time-depth graph of P-wave and S-wave picks. Line segments are straightline interpolations of model predictions at the observation depths. The times for zero depth, not shown, are given by hoffset divided by the velocity in the uppermost layer (see accompanying tables of velocities for specific values). 


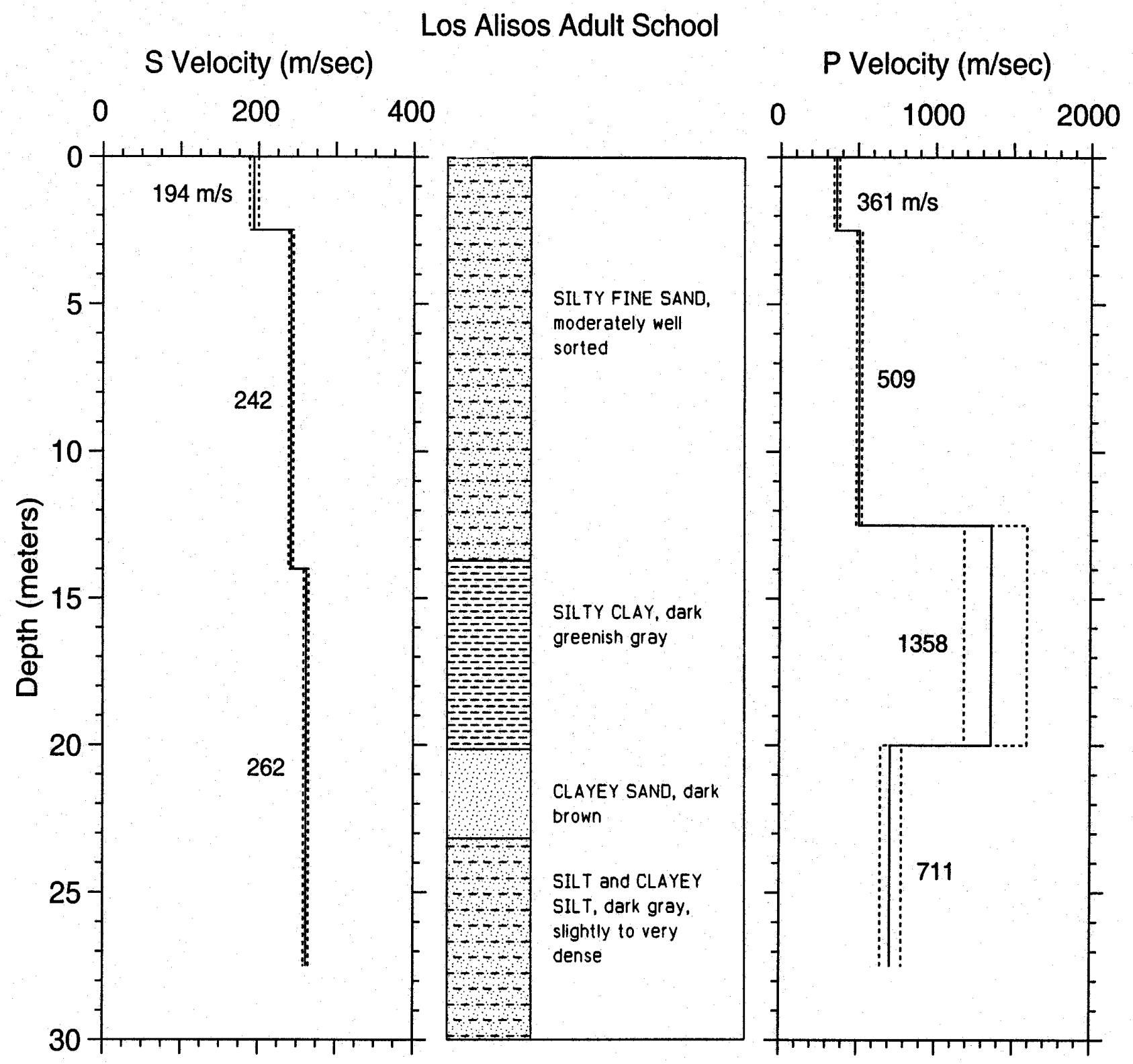

Figure A-40. S- and P-wave velocity profiles with dashed lines representing one standard deviation. Lithology is shown for correlation with velocities. 
ABLE A-15. S-wave arrival times and velocity summaries.

Location: Los Alisos: S

Coordinates:

hoffset $=2.00$ travel-time file: $\mathrm{P}: \backslash \mathrm{EXC} \backslash \mathrm{EXCS} . \mathrm{TT}$

$33.89560-118.08427$ Hole_Code: 302

nlayers $=3$

$\begin{array}{rrrcccc}\mathrm{d}(\mathrm{I}) & \mathrm{d}(\mathrm{ft}) & \mathrm{tsl}(s) & \operatorname{tvrt}(s) & \operatorname{vavg}(\mathrm{m} / \mathrm{s}) & \text { sig } & \mathrm{rsdl}(\mathrm{sec}) \\ 2.5 & 8.2 & 0.0163 & 0.0129 & 194 & 1 & -0.0002 \\ 5.0 & 16.4 & 0.0253 & 0.0232 & 215 & 1 & 0.0003 \\ 7.5 & 24.6 & 0.0345 & 0.0335 & 224 & 1 & -0.0003 \\ 10.0 & 32.8 & 0.0449 & 0.0439 & 228 & 1 & 0.0001 \\ 12.5 & 41.0 & 0.0554 & 0.0542 & 231 & 1 & 0.0005 \\ 15.0 & 49.2 & 0.0648 & 0.0642 & 234 & 1 & 0.0000 \\ 17.5 & 57.4 & 0.0743 & 0.0738 & 237 & 1 & 0.0000 \\ 20.0 & 65.6 & 0.0830 & 0.0833 & 240 & 1 & -0.0007 \\ 22.5 & 73.8 & 0.0936 & 0.0929 & 242 & 1 & 0.0003 \\ 25.0 & 82.0 & 0.1017 & 0.1024 & 244 & 1 & -0.0010 \\ 27.5 & 90.2 & 0.1132 & 0.1119 & 246 & 1 & 0.0009\end{array}$

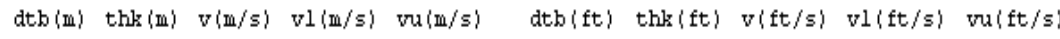

$\begin{array}{rrrrrrrrrr}2.5 & 2.5 & 194 & 188 & 200 & 8.2 & 8.2 & 636 & 618 & 656 \\ 14.0 & 11.5 & 242 & 239 & 245 & 45.9 & 37.7 & 794 & 784 & 804 \\ 27.5 & 13.5 & 262 & 259 & 265 & 90.2 & 44.3 & 859 & 849 & 870\end{array}$

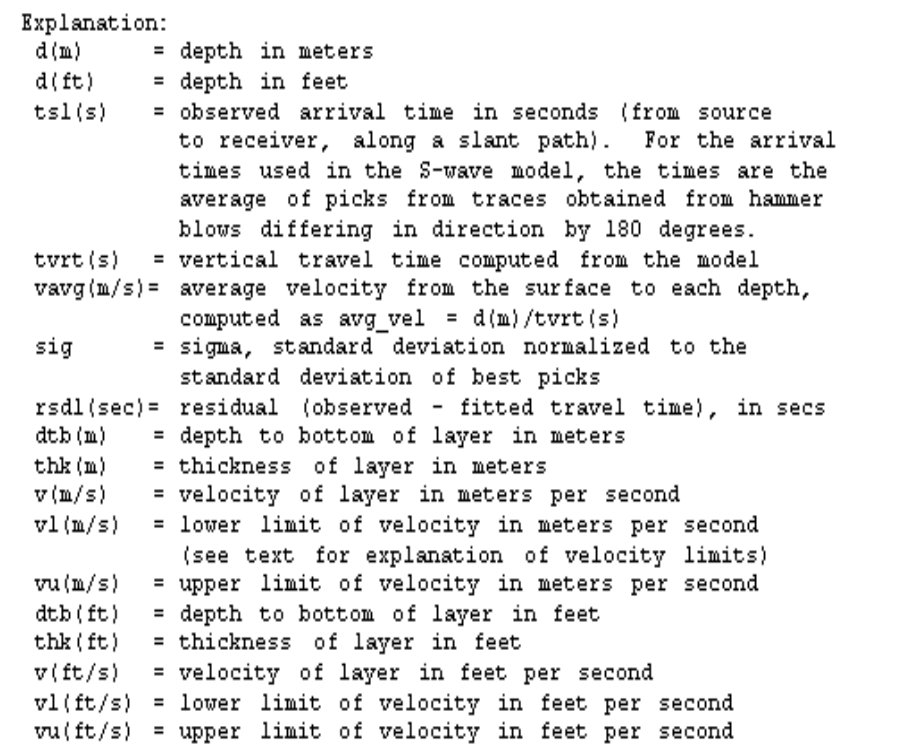


ABLE A-16. P-wave arrival times and velocity summaries.

Location: Los Alisos: p Coordinates: hoffset $=2.00$ travel-time file: $\mathrm{P}: \backslash \mathrm{EXC} \backslash \mathrm{EXCP} . \mathrm{T}$

$33.89560-118.08427$ Hole_Code: 302 nlayers $=4$

\begin{tabular}{|c|c|c|c|c|c|c|}
\hline II) & $\mathrm{d}(\mathrm{ft})$ & $1(s)$ & tvrt & $\operatorname{vavg}(\mathbf{m} / 5)$ & sig & \\
\hline 2.5 & 8.2 & 0.0093 & 0.0069 & 361 & 2 & 0.0005 \\
\hline 5.0 & 16.4 & 0.0116 & 0.0118 & 422 & 2 & -0.0011 \\
\hline 7.5 & 24.6 & 0.0182 & 0.0167 & 448 & 3 & 0.0009 \\
\hline 0.0 & 32.8 & 0.0222 & 0.0217 & 462 & 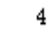 & 0.0001 \\
\hline .5 & 41.0 & 0.0270 & 0.0266 & 470 & 3 & 0.0001 \\
\hline 0 & 49. & 0.02 & & 528 & 3 & -0.0001 \\
\hline .5 & 57.4 & 0.0306 & 0.0303 & 578 & 3 & 0.0002 \\
\hline .0 & 65.6 & 0.03 & & 623 & & -0.0 \\
\hline .5 & 73.8 & 0.0358 & 0.0 & 63 & & 0.0001 \\
\hline .0 & 82.0 & 0.0392 & 0.0391 & 639 & 2 & 0.0000 \\
\hline 1.5 & 90.2 & 0.0426 & 0.0426 & 645 & & -0.0001 \\
\hline
\end{tabular}

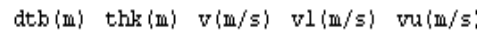

$\begin{array}{rrrrrrrrrr}\text { dtth (ii) } & \text { thk }(\mathrm{m}) & \mathrm{v}(\mathrm{m} / \mathrm{s}) & \mathrm{vl}(\mathrm{m} / \mathrm{s}) & \mathrm{vu}(\mathrm{m} / \mathrm{s}) & \mathrm{dth}(\mathrm{ft}) & \mathrm{thk}(\mathrm{ft}) & \mathrm{v}(\mathrm{ft} / \mathrm{s}) & \mathrm{vl}(\mathrm{ft} / \mathrm{s}) & \mathrm{vu}(\mathrm{ft} / \mathrm{s}) \\ 2.5 & 2.5 & 361 & 344 & 380 & 8.2 & 8.2 & 1186 & 1129 & 1248 \\ 12.5 & 10.0 & 509 & 491 & 527 & 41.0 & 32.8 & 1669 & 1611 & 1730 \\ 20.0 & 7.5 & 1358 & 1185 & 1590 & 65.6 & 24.6 & 4456 & 3889 & 5218 \\ 27.5 & 7.5 & 711 & 650 & 785 & 90.2 & 24.6 & 2334 & 2133 & 2575\end{array}$

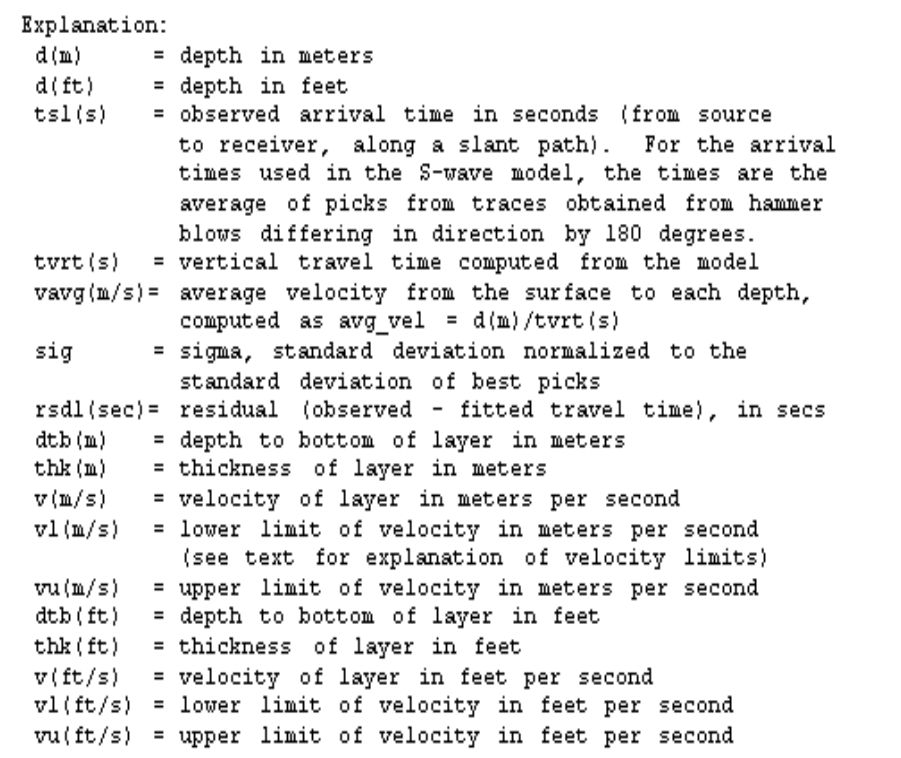




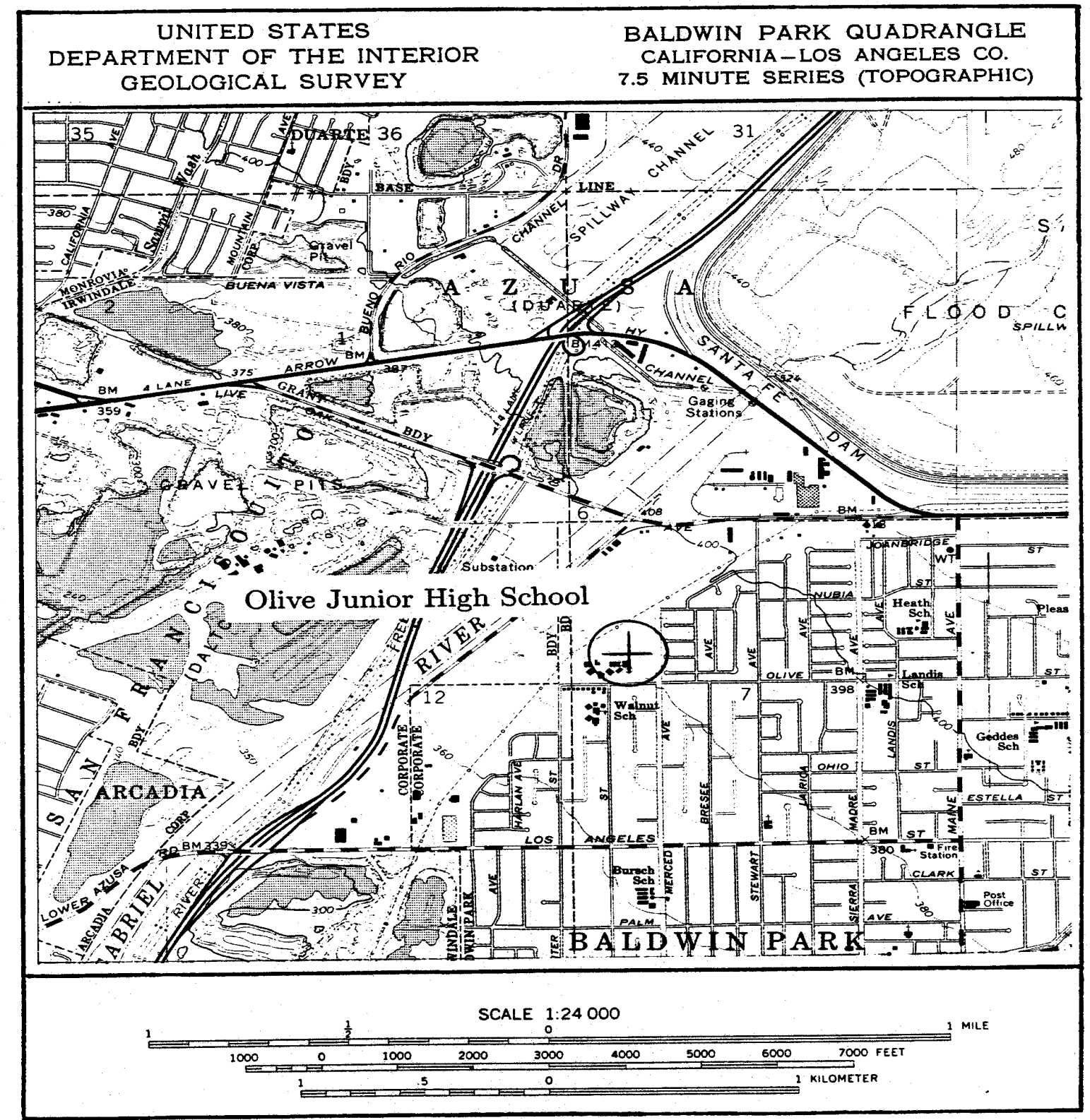

Figure A-41. Site location map for the borehole at Olive Junior High School. The accelerograph is located approximately 46 meters from the borehole. 


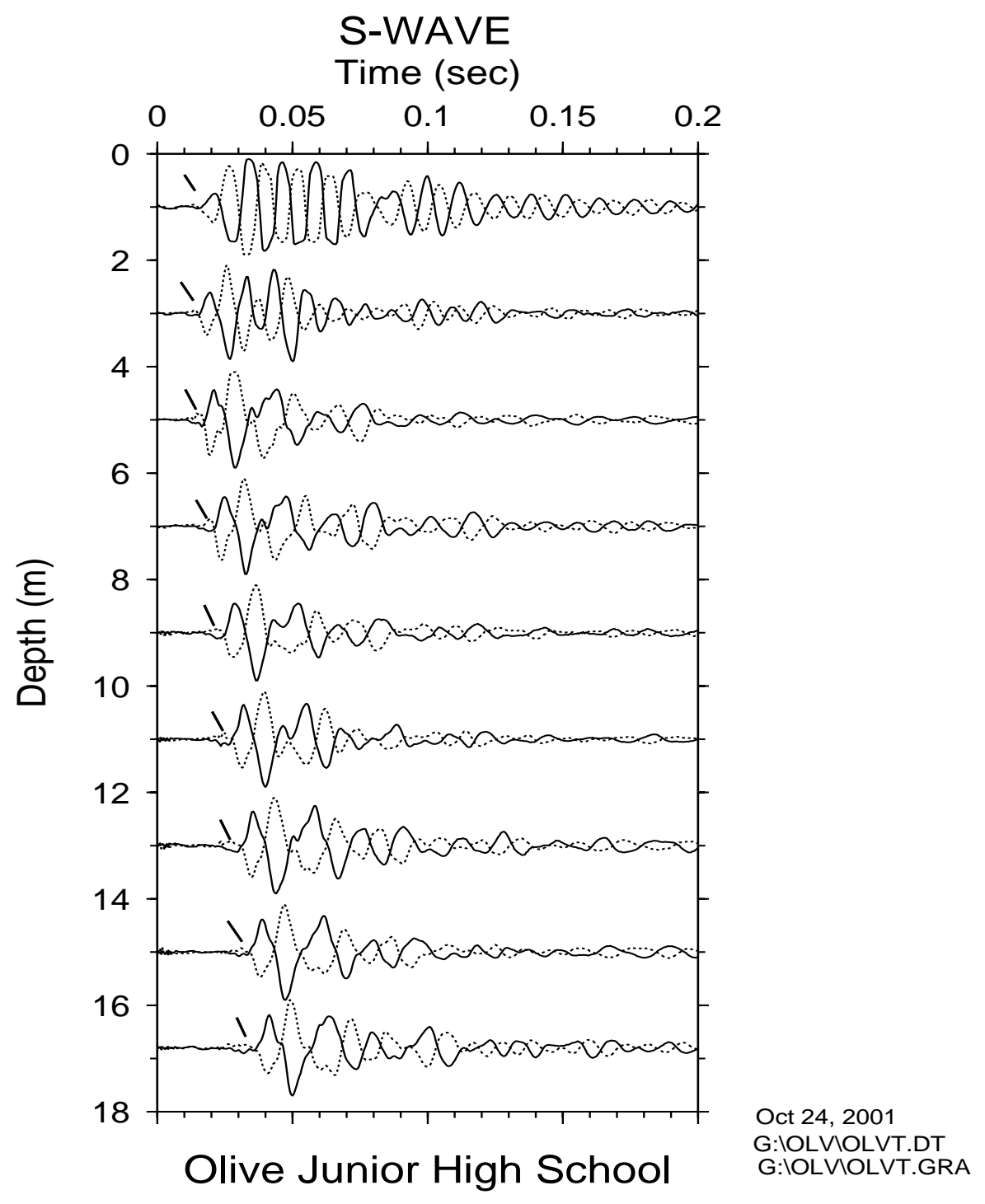

Figure A-42. Horizontal component record section (from impacts in opposite directions) superimposed for identification of S-wave onset. Approximate S-wave time picks are indicated by the hatch marks. 


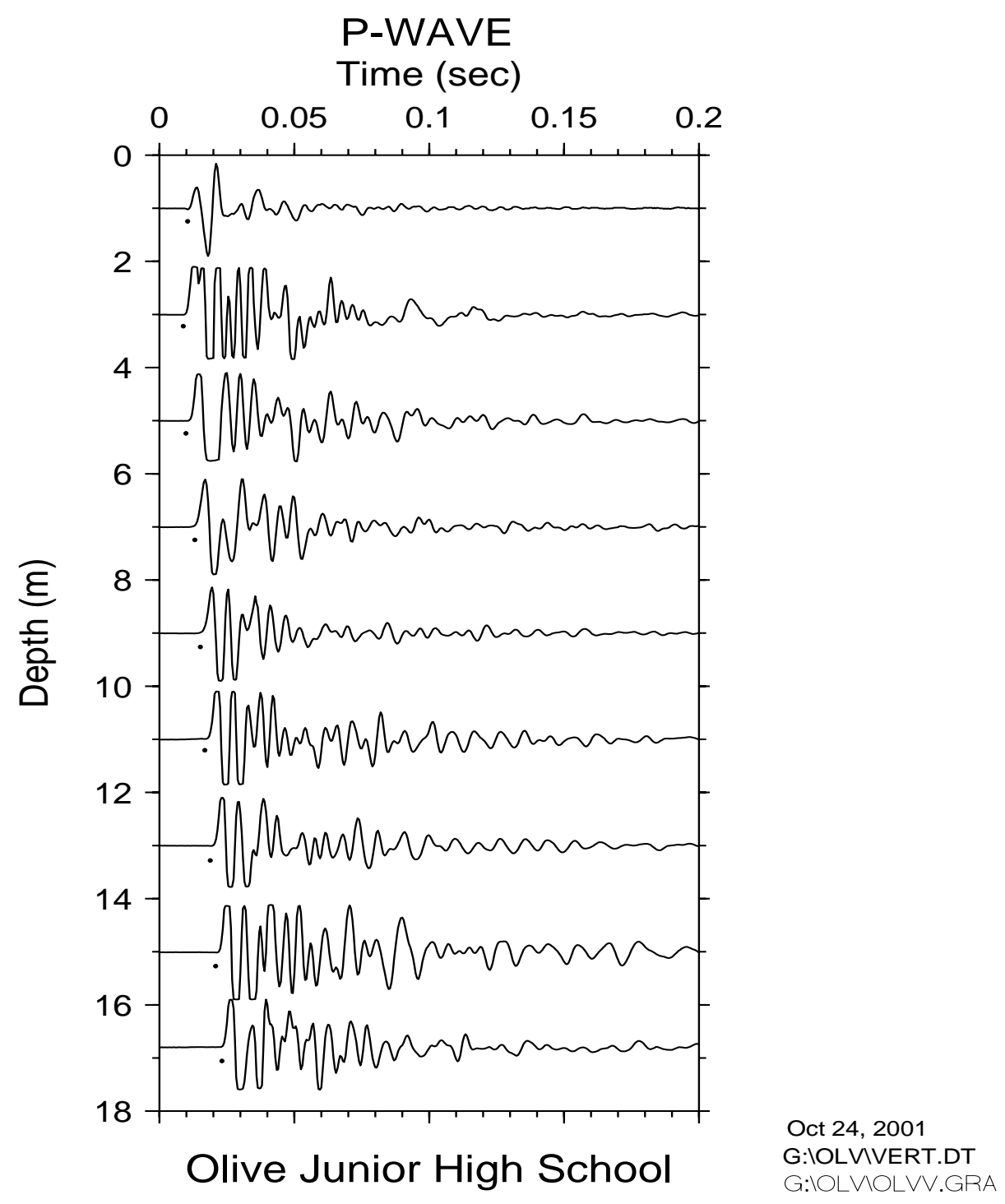

Figure A-43. Vertical component record section. Approximate P-wave arrivals are indicated by the dots. 


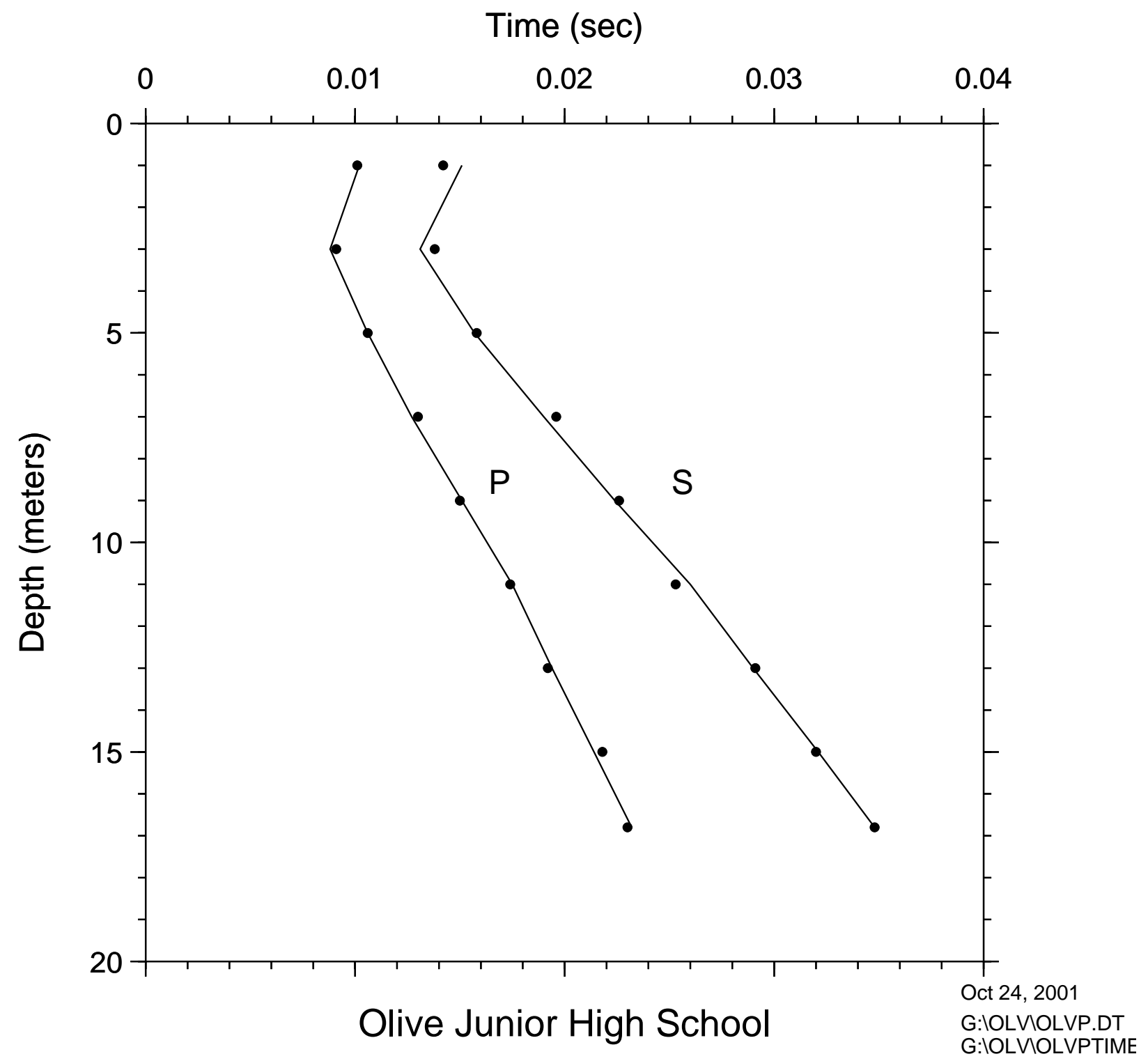

Figure A-44. Time-depth graph of P-wave and S-wave picks. Line segments are straightline interpolations of model predictions at the observation depths. The times for zero depth, not shown, are given by hoffset divided by the velocity in the uppermost layer (see accompanying tables of velocities for specific values). 


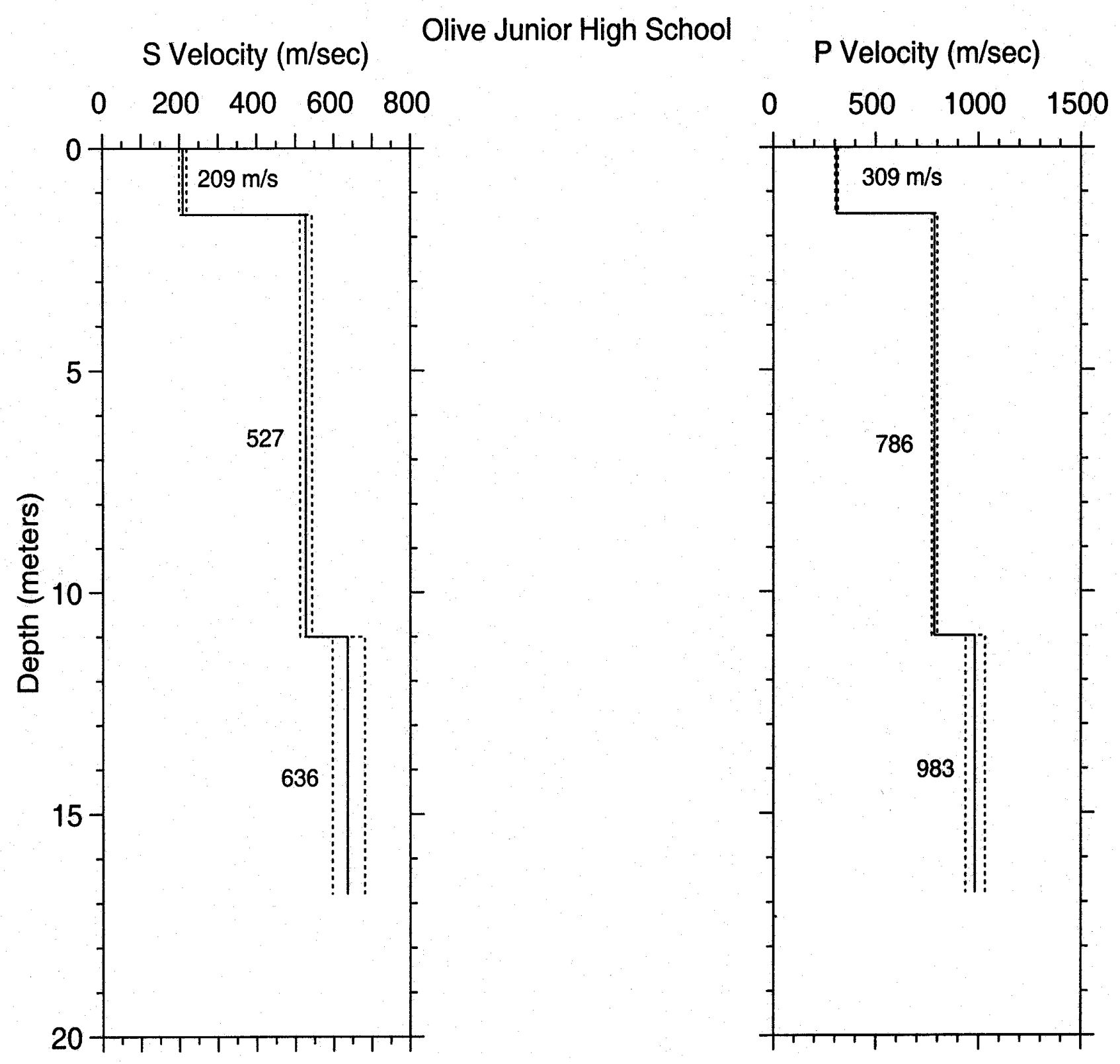

Figure A-45. S- and P-wave velocity profiles with dashed lines representing one standard deviation. Lithology is not available from this borehole. 
ABLE A-17. S-wave arrival times and velocity summaries.

Location: Olive Jr. High School: $\$$ Coordinates:
hoffset $=3.00$ travel-time file: G: $\backslash$ OLV 0 OUS.TT

$\begin{array}{rrrrrrr}\mathrm{d}(\mathrm{II}) & \mathrm{d}(\mathrm{ft}) & \mathrm{tsl}(s) & \mathrm{tvrt}(s) & \text { vavg}(\mathrm{m} / \mathrm{s}) & \text { sig } & \mathrm{rsdl}(\mathrm{sec}) \\ 1.0 & 3.3 & 0.0142 & 0.0048 & 209 & 2 & -0.0009 \\ 3.0 & 9.8 & 0.0138 & 0.0100 & 299 & 1 & 0.0007 \\ 5.0 & 16.4 & 0.0158 & 0.0138 & 362 & 1 & 0.0001 \\ 7.0 & 23.0 & 0.0196 & 0.0176 & 397 & 1 & 0.0006 \\ 9.0 & 29.5 & 0.0226 & 0.0214 & 420 & 1 & 0.0001 \\ 11.0 & 36.1 & 0.0253 & 0.0252 & 436 & 1 & -0.0008 \\ 13.0 & 42.7 & 0.0291 & 0.0283 & 459 & 1 & 0.0001 \\ 15.0 & 49.2 & 0.0320 & 0.0315 & 476 & 1 & 0.0000 \\ 16.8 & 55.1 & 0.0348 & 0.0343 & 489 & 1 & 0.0000\end{array}$

$34.10073-117.97409$ Hole_Code: 303 nlayers $=3$

dtb(ii) $\operatorname{thk}(\mathrm{m}) \quad \mathrm{v}(\mathrm{m} / \mathrm{s}) \quad \mathrm{vl}(\mathrm{m} / \mathrm{s}) \quad \mathrm{vu}(\mathrm{m} / \mathrm{s}) \quad \mathrm{dtb}(\mathrm{ft}) \operatorname{thk}(\mathrm{ft}) \quad \mathrm{v}(\mathrm{ft} / \mathrm{s}) \quad \mathrm{vl}(\mathrm{ft} / \mathrm{s}) \quad \mathrm{vu}(\mathrm{ft} / \mathrm{s})$

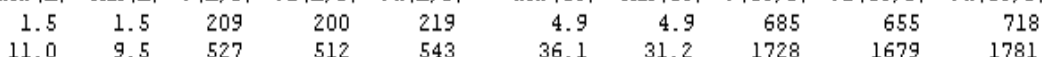

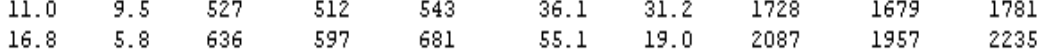

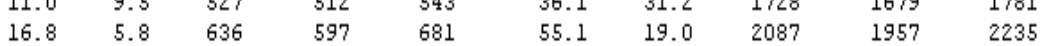

Bxplanation:

$d(\mathbb{i}) \quad=$ depth in meter

$d(\mathrm{ft})=$ depth in feet

tslis) = observed arrival time in seconds (from source to receiver, along a slant path). For the arrival times used in the s-wave model, the times are the average of picks from traces obtained from haminer blows differing in direction by 180 degrees.

tvrt (s) = vertical travel time computed from the model

$\operatorname{vavg}(i \mathrm{~m} / s)=$ average velocity from the surface to each depth, computed as avg vel $=d(m) /$ tvrt $(s)$

= sigma, standard deviation normalized to the standard deviation of best picks

rsdl (sec) = residual (observed - fitted travel time), in secs

$d \operatorname{dt}(\mathrm{II})=$ depth to bott

thk $\langle\mathrm{II}\rangle=$ thickness of layer in meters

$\mathrm{v}(\mathrm{Ii} / \mathrm{s})=$ velocity of layer in meters per second

$\mathrm{vl}(\mathrm{m} / \mathrm{s})=$ lower limit of velocity in meters per second (see text for explanation of velocity limits)

$\mathrm{vu}(\mathrm{i} / \mathrm{s})=$ upper limit of velocity in meters per second

dtb $(\mathrm{ft})=$ depth to bottom of layer in feet

thl $(\mathrm{ft})=$ thickness of layer in feet

$\mathrm{v}(\mathrm{ft} / \mathrm{s})=$ velocity of layer in feet per second

$\mathrm{vl}(\mathrm{ft} / \mathrm{s})=$ lower limit of velocity in feet per second

$\mathrm{vu}(\mathrm{ft} / \mathrm{s})=$ upper limit of velocity in feet per second 
ABLE A-18. P-wave arrival times and velocity summaries.

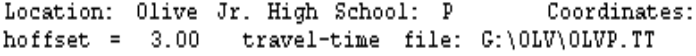

$\begin{array}{rrrccrr}\mathrm{d}(\mathrm{II}) & \mathrm{d}(\mathrm{ft}) & \mathrm{tsl}(s) & \text { tvrt }(s) & \text { vavg }(\mathrm{m} / \mathrm{s}) & \text { sig } & \mathrm{rsdl}(\mathrm{sec}) \\ 1.0 & 3.3 & 0.0101 & 0.0032 & 309 & 1 & -0.0001 \\ 3.0 & 9.8 & 0.0091 & 0.0068 & 444 & 1 & 0.0002 \\ 5.0 & 16.4 & 0.0106 & 0.0093 & 537 & 1 & 0.0000 \\ 7.0 & 23.0 & 0.0130 & 0.0119 & 591 & 1 & 0.0002 \\ 9.0 & 29.5 & 0.0150 & 0.0144 & 625 & 1 & -0.0001 \\ 11.0 & 36.1 & 0.0174 & 0.0169 & 649 & 1 & -0.0001 \\ 13.0 & 42.7 & 0.0192 & 0.0190 & 685 & 1 & -0.0002 \\ 15.0 & 49.2 & 0.0218 & 0.0210 & 714 & 1 & 0.0004 \\ 16.8 & 55.1 & 0.0230 & 0.0228 & 736 & 1 & -0.0002\end{array}$

$34.10073-117.97409$ Hole_Code: 303 nlayers $=3$

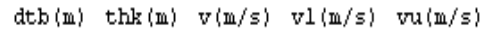

$\mathrm{dtb}(\mathrm{ft}) \quad \mathrm{thl}(\mathrm{ft}) \quad \mathrm{v}(\mathrm{ft} / \mathrm{s}) \quad \mathrm{vl}(\mathrm{ft} / \mathrm{s}) \quad \mathrm{vu}(\mathrm{ft} / \mathrm{s})$

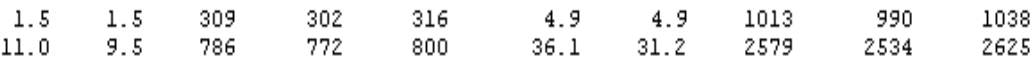

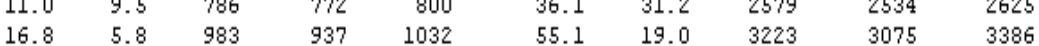

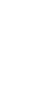

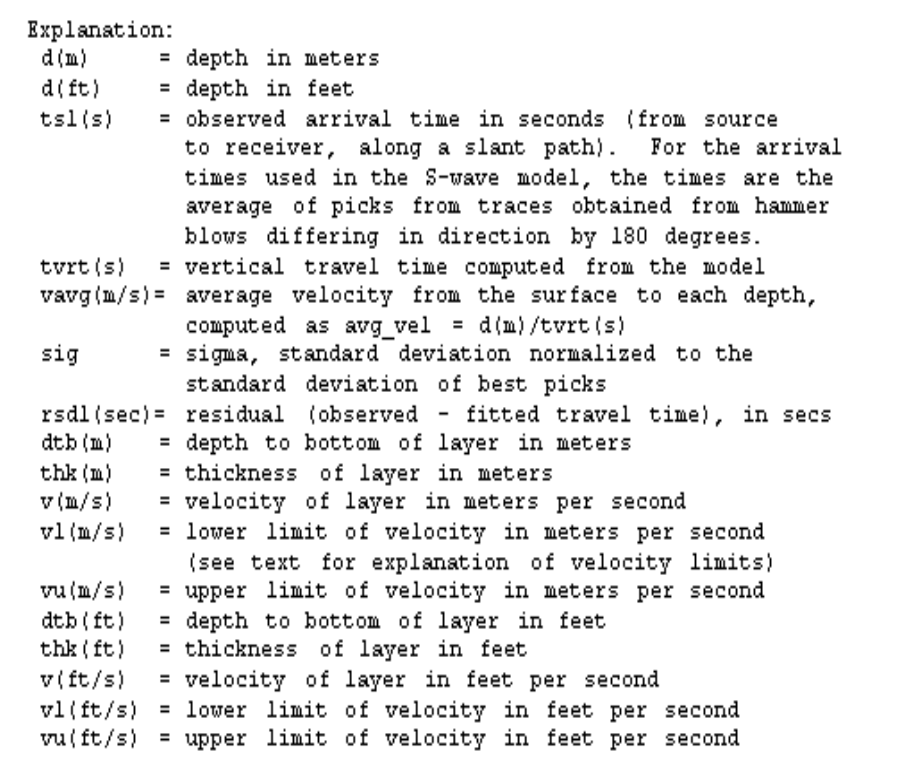






Figure A-46. Site location map for the borehole at San Bernardino Main Fire Station. 


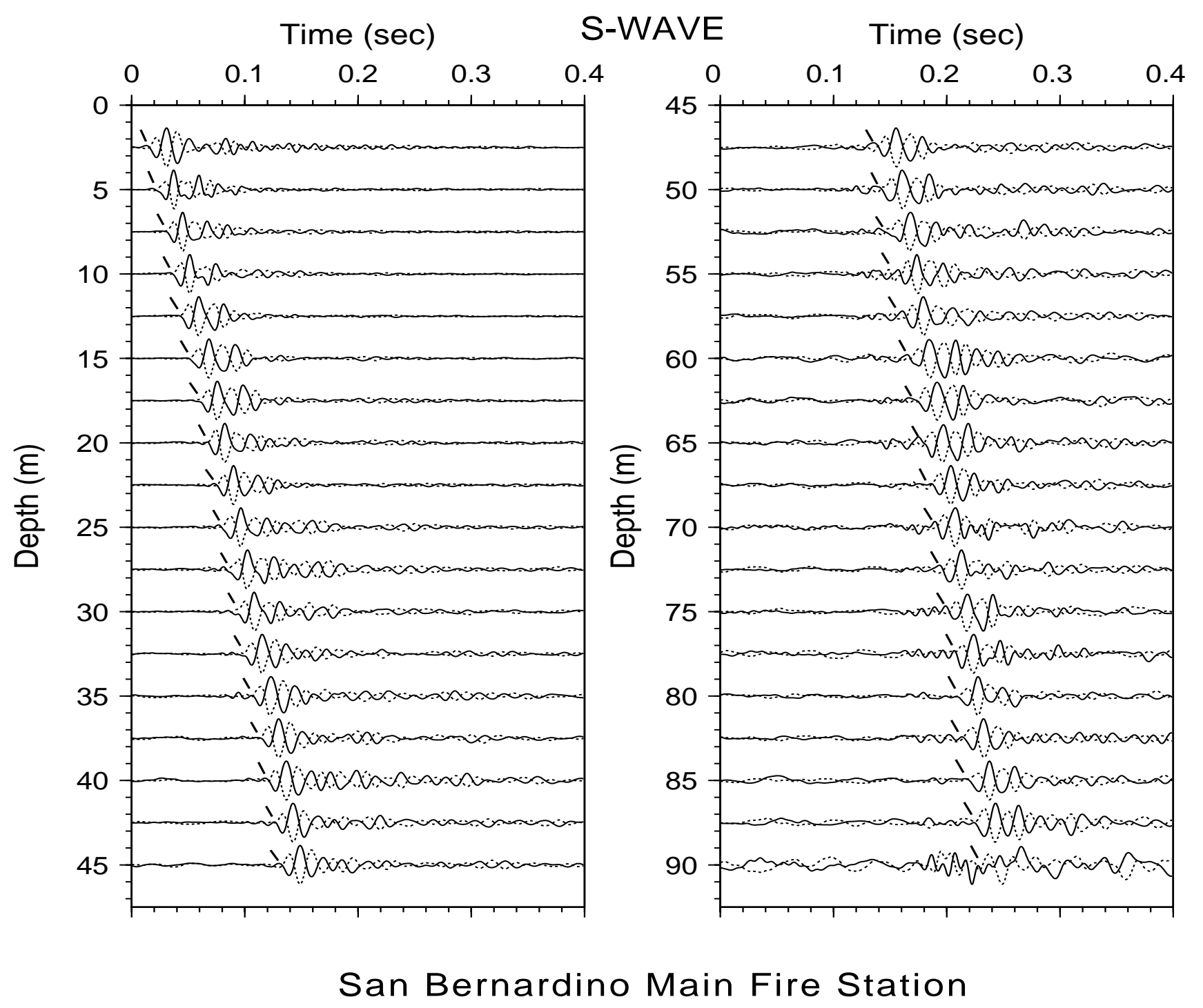

Figure A-47. Horizontal component record section (from impacts in opposite directions) superimposed for identification of S-wave onset. Approximate S-wave time picks are indicated by the hatch marks. 


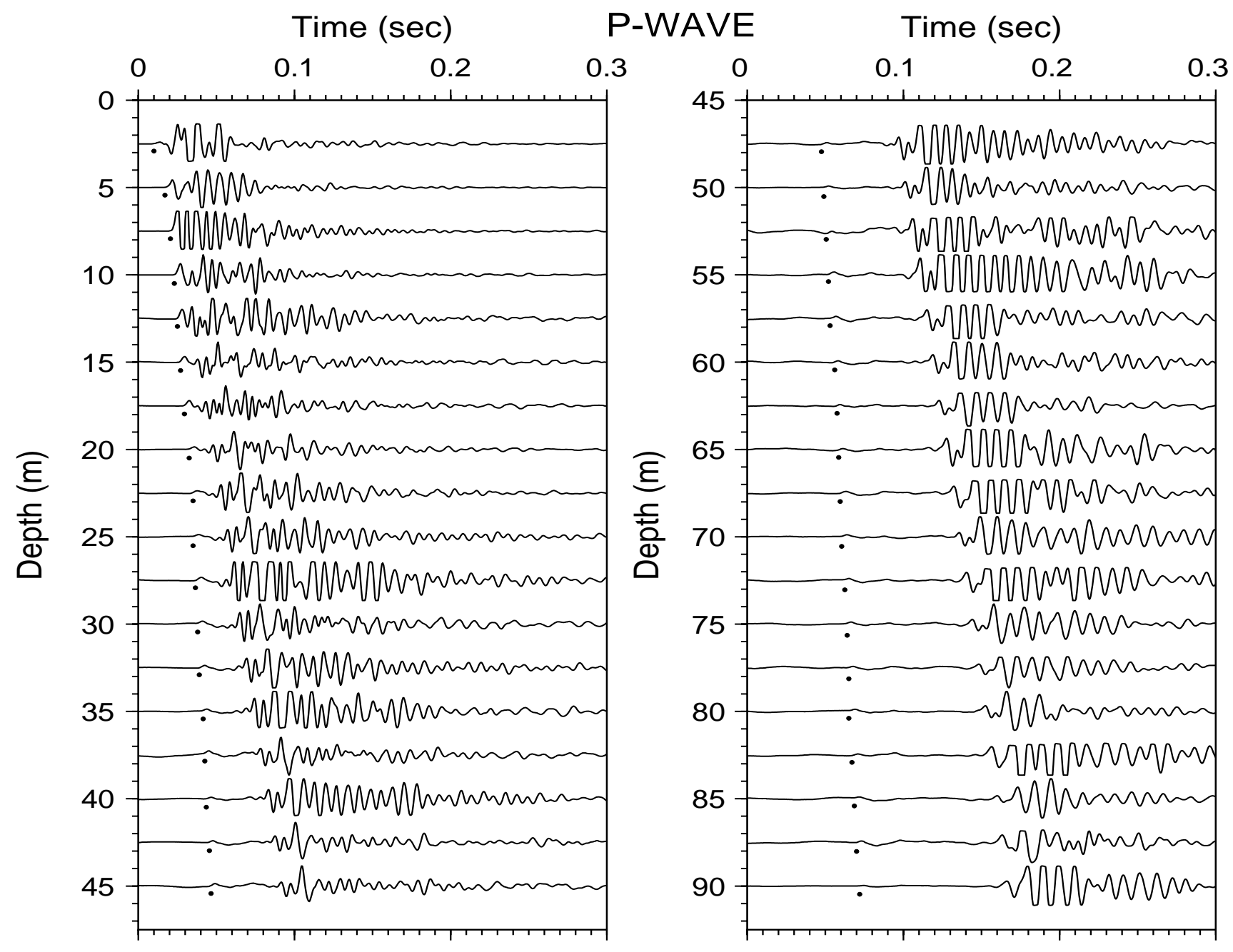

Oct 24, 2001

F:ISB1ISB1VERT.DT

F:ISB1ISB1V45.GRA

San Bernardino Main Fire Station

Oct 24, 2001

F:ISB1ISB1V.DT

F:ISB1ISB1V4.GR

Figure A-48. Vertical component record section. Approximate P-wave arrivals are indicated by the dots. 


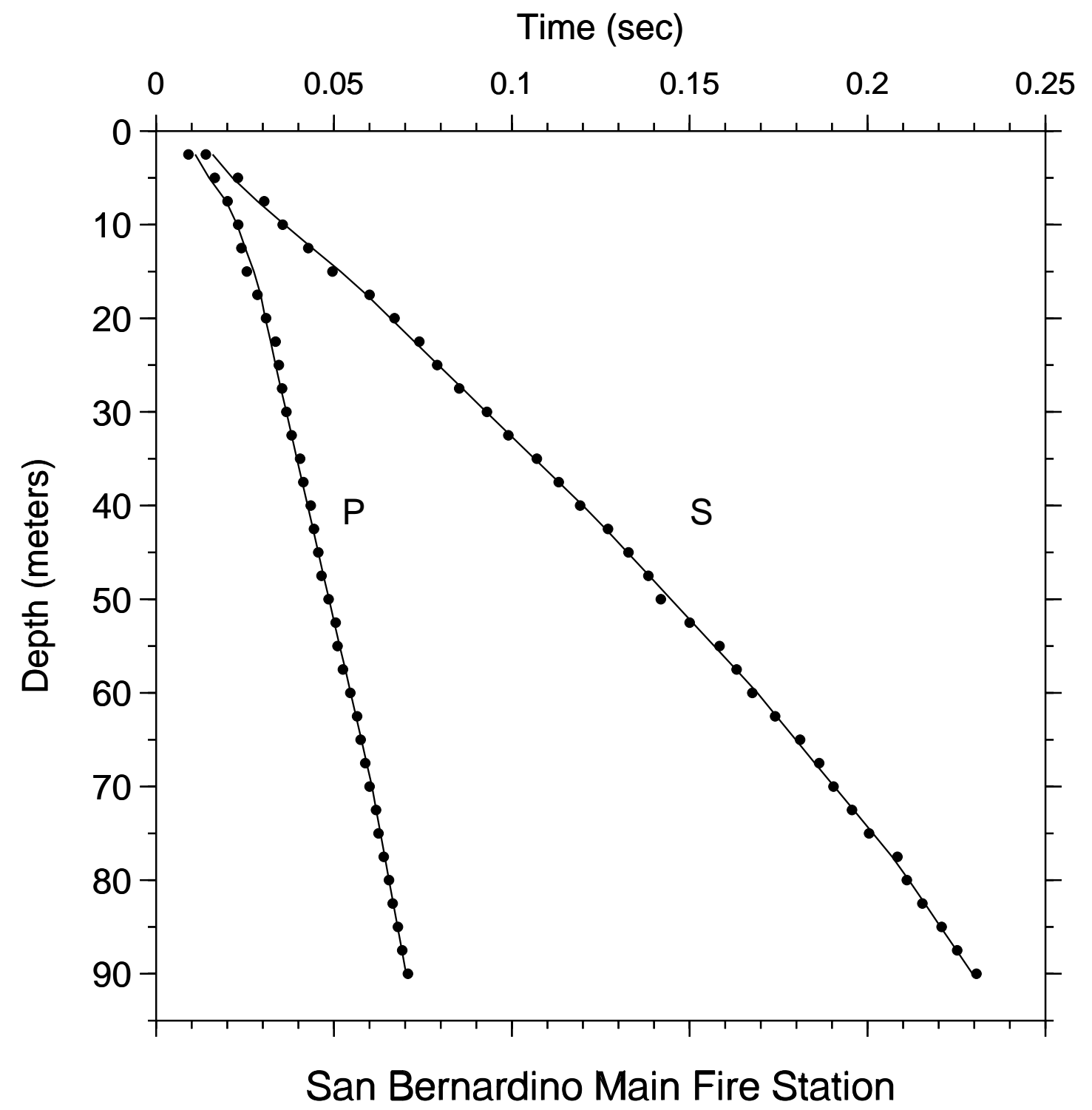

- d_obs

- d obs

Figure A-49. Time-depth graph of P-wave and S-wave picks. Line segments are straightline interpolations of model predictions at the observation depths. The times for zero depth, not shown, are given by hoffset divided by the velocity in the uppermost layer (see accompanying tables of velocities for specific values). 


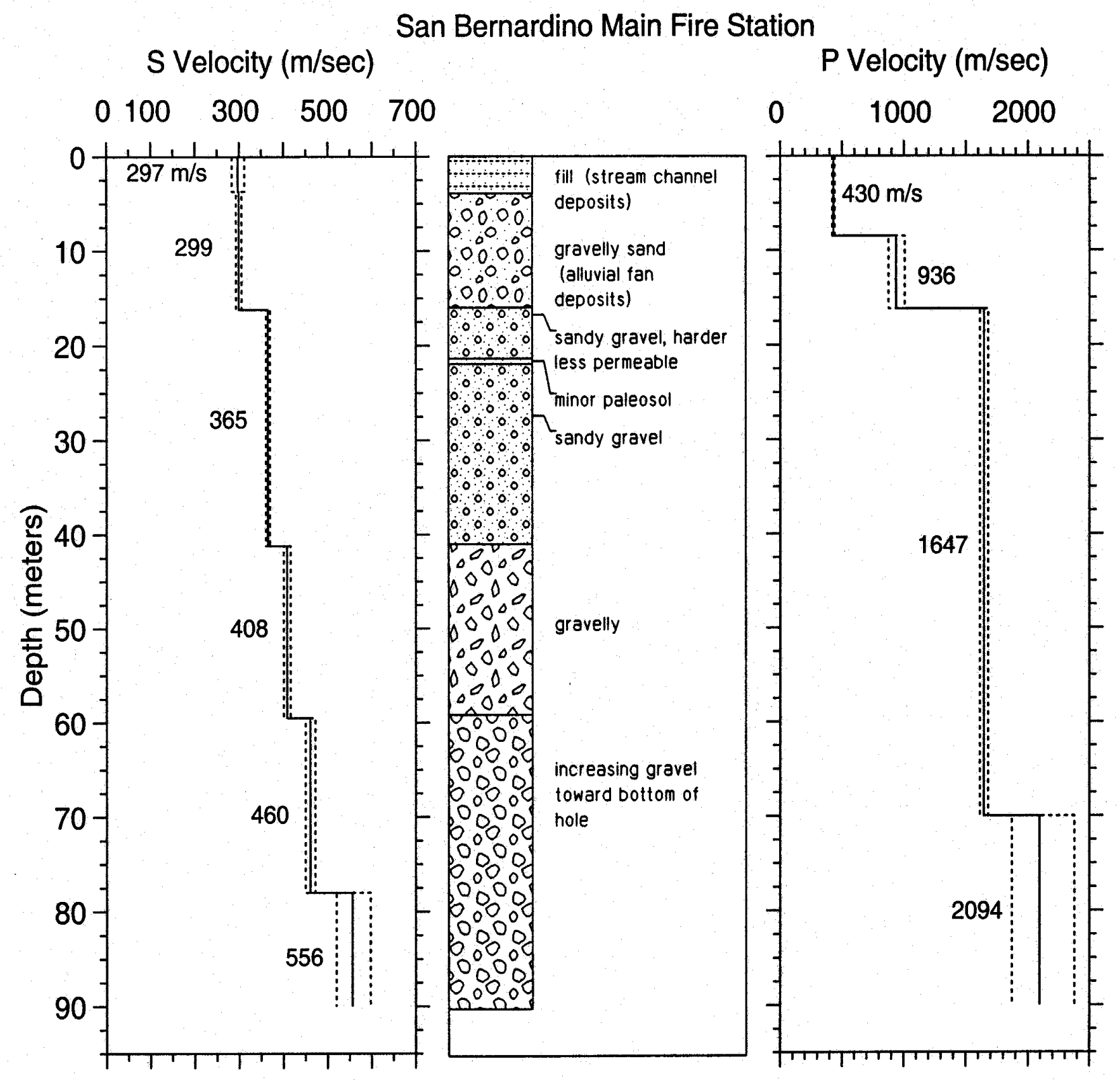

Figure A-50. S- and P-wave velocity profiles with dashed lines representing one standard deviation. Lithology is shown for correlation with velocities. 
ABLB A-19. S-wave arrival times and velocity suminaries.

Location: San Bernardino Fire Station: S Coordinates: hoffset $=4.00$ travel-time file: $\mathrm{F}: \backslash$ SB $\backslash$ SB1S.TT

\begin{tabular}{|c|c|c|c|c|c|c|}
\hline in) & $d(f t)$ & $t \leq 1(s)$ & tvrt (s) & $f(i n / s)$ & sig & \\
\hline & 8.2 & 0.0140 & 0.0084 & 297 & 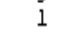 & -0.0019 \\
\hline 5.0 & 16.4 & 0.0230 & 0.0168 & 298 & 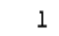 & 0.0015 \\
\hline 7.5 & 24.6 & 0.0304 & 0.0252 & 298 & 1 & 0.0019 \\
\hline 10.0 & 32.8 & 0.0356 & 0.0335 & 298 & 1 & -0.0005 \\
\hline 12.5 & 41.0 & 0.0428 & 0.0419 & 298 & 1 & -0.0011 \\
\hline 15.0 & 49.2 & 0.0496 & 0.0503 & 299 & 1 & -0.0024 \\
\hline 17.5 & 57.4 & 0.0600 & 0.0578 & 303 & + & 0.0008 \\
\hline 0.0 & 65.6 & 0.0670 & 0.0647 & 200 & & 0.0011 \\
\hline .5 & 73.8 & 0.0740 & 0.0715 & 315 & 1 & 0.0015 \\
\hline .0 & 82.0 & 0.0790 & 0.0784 & & 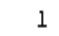 & -0.0003 \\
\hline .5 & 90.2 & 0.0852 & .0852 & 323 & 2 & -0.0009 \\
\hline 30.0 & 98.4 & 0.0930 & 0.0921 & 326 & 1 & 0.0002 \\
\hline 32.5 & 106.6 & 0.0990 & 0.0989 & 329 & 1 & -0.0006 \\
\hline 5.0 & 114.8 & 0.1070 & 0.1058 & 331 & 1 & 0.0006 \\
\hline 37.5 & 123.0 & 0.1132 & 0.1126 & 333 & 1 & 0.0000 \\
\hline 40.0 & 131.2 & 0.1192 & 0.1195 & 335 & 1 & -0.0008 \\
\hline 42.5 & 139.4 & 0.1270 & 0.1259 & 337 & 1 & 0.0005 \\
\hline .0 & 147.6 & 0.1328 & 0. & 341 & 1 & 0.0003 \\
\hline 7.5 & 155.8 & 0.1384 & 0.1382 & 34 & 1 & -0.0002 \\
\hline 50.0 & 164.0 & 0.1419 & & 34 & 2 & -0.0028 \\
\hline 2.5 & 172.2 & 0.1500 & 05 & 34 & 2 & -0.0008 \\
\hline 55.0 & 180.4 & 0.1584 & 0.1566 & 351 & 1 & 0.0015 \\
\hline 7.5 & 188.6 & 0.1632 & 0.1627 & 353 & 1 & 0.0002 \\
\hline 0.0 & 196.9 & 0.1676 & 0 & 35 & 1 & -0.0014 \\
\hline 52.5 & 205.1 & 0.1740 & 0.1 & 359 & 1 & -0.0004 \\
\hline 65.0 & 213.3 & 0.1810 & 0.1796 & 362 & & 0.0012 \\
\hline 67.5 & 221.5 & 0.1864 & 0.1850 & 365 & 1 & 0.0012 \\
\hline 70.0 & 229.7 & 0.1904 & 0.1904 & 368 & 1 & -0.0002 \\
\hline 72.5 & 237.9 & 0.1956 & & 97 & & -0.0005 \\
\hline 75.0 & 246.1 & 0.2004 & 0.2013 & 373 & 1 & -0.0011 \\
\hline 7.5 & 254.3 & & & & & \\
\hline 80.0 & 262.5 & 0.2110 & 0.2114 & 378 & 1 & -0.0006 \\
\hline 82.5 & 270.7 & 0.2154 & & 382 & & \\
\hline & & & & & & \\
\hline & 287.1 & 0.2 & 0.2249 & 389 & , & 0.0 \\
\hline & & & & & & 000 \\
\hline
\end{tabular}

$34.10534-117.28201$ Hole_Code: 305

nlayers $=6$

dtb (ii) thk (ii) $\mathrm{v}(\mathrm{m} / \mathrm{s}) \quad \mathrm{vl}(\mathrm{II} / \mathrm{s}) \quad \mathrm{vu}(\mathrm{m} / \mathrm{s})$ $\begin{array}{lllll}3.7 & 3.7 & 297 & 284 & 312\end{array}$ $\begin{array}{llll}16.2 & 12.5 & 299 & 293\end{array}$ $\begin{array}{lllll}41.2 & 25.0 & 365 & 360 & 370\end{array}$

$\begin{array}{ll}41.2 & 25.0 \\ 59.5 & 18.3\end{array}$

$78.0 \quad 18.5$

$78.0 \quad 18.5$ $50.0-12.0-55$ 
ABLB A-20. P-wave arrival times and velocity suminaries.

Location: San Bernardino Fire Station: P Coordinates: hoffset $=4.00$ travel-time file: $\mathrm{F}: \backslash$ SB $\backslash$ SB1P.TT

\begin{tabular}{|c|c|c|c|c|c|c|}
\hline iil) & $d(f t)$ & $1(s)$ & tvrt (s) & $\operatorname{vavg}(\mathrm{I} / \mathrm{s})$ & sig & \\
\hline 2.5 & 8.2 & 0.0091 & 0.0058 & 430 & 1 & -0.0019 \\
\hline 5.0 & 16.4 & 0.0165 & 0.0116 & 430 & 1 & 0.0016 \\
\hline 7.5 & 24.6 & 0.0201 & 0.0174 & 430 & 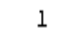 & 0.0003 \\
\hline 10.0 & 32.8 & 0.0231 & 0.0214 & 468 & 1 & 0.0002 \\
\hline 12.5 & 41.0 & 0.0240 & 0.0240 & 520 & 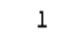 & -0.0010 \\
\hline 0 & 49.2 & 0.0255 & 0.0267 & 562 & 1 & -0.0020 \\
\hline .5 & 57.4 & 0.0285 & 0.0288 & 608 & 1 & -0.0008 \\
\hline .0 & 65.6 & 0.0309 & 0.0303 & 660 & 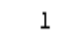 & 0.0002 \\
\hline .5 & 73.8 & 0.0336 & 0.0318 & 707 & 1 & 0.0015 \\
\hline 0 & 82.0 & 0.0345 & 0.0333 & 750 & - & 0.0009 \\
\hline .5 & 90.2 & 0.0354 & 0.0349 & 789 & 1 & 0.0003 \\
\hline .0 & 98.4 & 0.0366 & 0.0364 & 25 & & 0.0000 \\
\hline .5 & 106.6 & 0.0381 & 0.0379 & 858 & & 0.0000 \\
\hline 0 & 114.8 & 0.0405 & 0.0 & 8 & & \\
\hline .5 & 123.0 & 0.0414 & 0.0 & 91 & & 0.0004 \\
\hline 0.0 & 131.2 & 0.0435 & 0.0424 & 96 & 3 & 0.0009 \\
\hline 42.5 & 139.4 & 0.0444 & 0.0440 & 967 & 1 & 0.0003 \\
\hline 5.0 & 147.6 & 0.0456 & 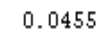 & $98:$ & & \\
\hline 47.5 & 155.8 & 0.0 & 0 & & 3 & -0 . \\
\hline 0.0 & 164.0 & 0.0485 & .0 & 31 & 4 & -0.0001 \\
\hline 52.5 & 172.2 & 0.0505 & 0. & & 3 & \\
\hline 5.0 & 180.4 & 0.0510 & 0.0516 & 1067 & 1 & -0.0006 \\
\hline 57.5 & 188.6 & 0.0525 & 0.0531 & 1083 & 3 & -0.0006 \\
\hline 0.0 & 196.9 & 0.0546 & 0. & 199 & 3 & 0.0000 \\
\hline 62.5 & 205.1 & 0.0565 & 0.0561 & 1114 & 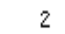 & 0.0003 \\
\hline 5.0 & 213.3 & 0.0575 & 0. & 28 & 2 & -0.0002 \\
\hline 77.5 & 221.5 & 0.0588 & 0.0591 & 1141 & 2 & -0.0004 \\
\hline 70.0 & 229.7 & & & & & \\
\hline 72.5 & 237.9 & & & & 2 & -0 \\
\hline 5.0 & 246.1 & & & & & \\
\hline 77.5 & 254.3 & & & & & \\
\hline 0.0 & 262.5 & 0.0655 & 0.0654 & 1223 & 2 & 0.0000 \\
\hline 82. & & & & & & \\
\hline 5. & 278.9 & 0.8 & 0.0 & 1253 & 2 & \\
\hline 87. & 287 & & & & 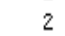 & \\
\hline 10. & 295.3 & 0.0708 & 0.0702 & 1282 & 3 & 0.0005 \\
\hline
\end{tabular}

$34.10534-117.28201$ Hole_Code: 305 nlayers $=4$

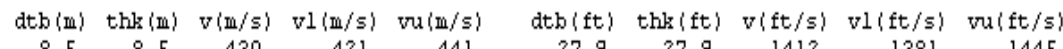

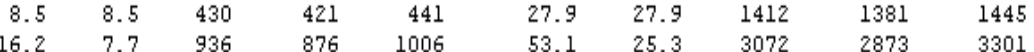
$\begin{array}{rrrrrrrrrr}16.2 & 7.7 & 936 & 876 & 1006 & 53.1 & 25.3 & 3072 & 2873 & 3301 \\ 70.0 & 53.8 & 1647 & 1614 & 1680 & 229.7 & 176.5 & 5402 & 5295 & 5513\end{array}$ $\begin{array}{rrrrrrrrrr}70.0 & 53.8 & 1647 & 1614 & 1680 & 229.7 & 176.5 & 5402 & 5295 & 5513 \\ 90.0 & 20.0 & 2094 & 1870 & 2378 & 295.3 & 65.6 & 6870 & 6136 & 7803\end{array}$

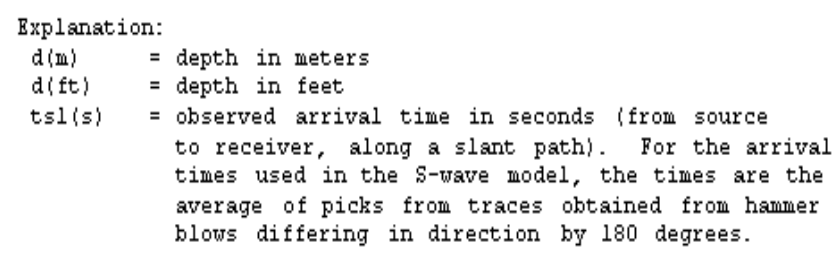




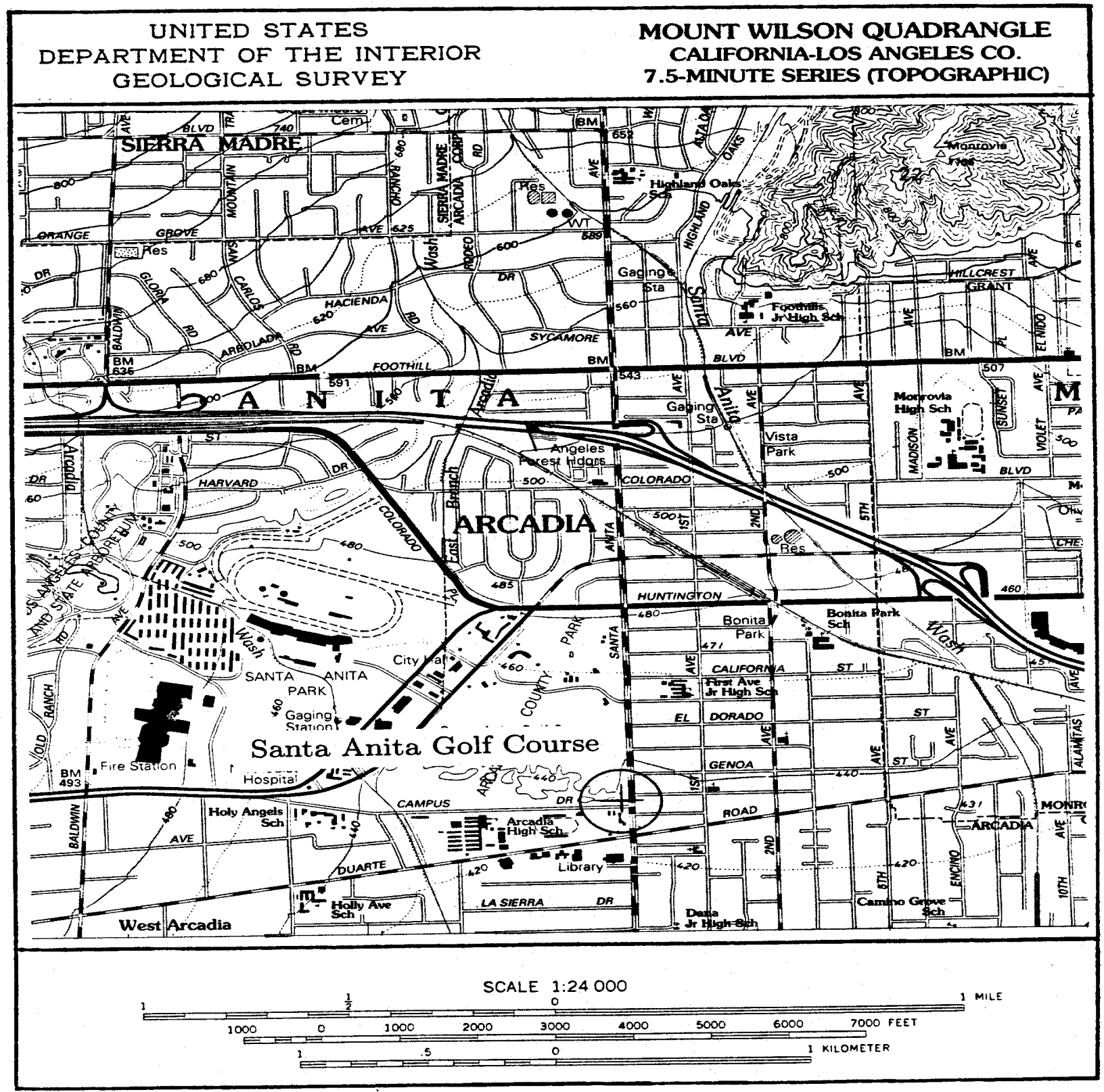

Figure A-51. Site location map for the borehole at Santa Anita Golf Course. 


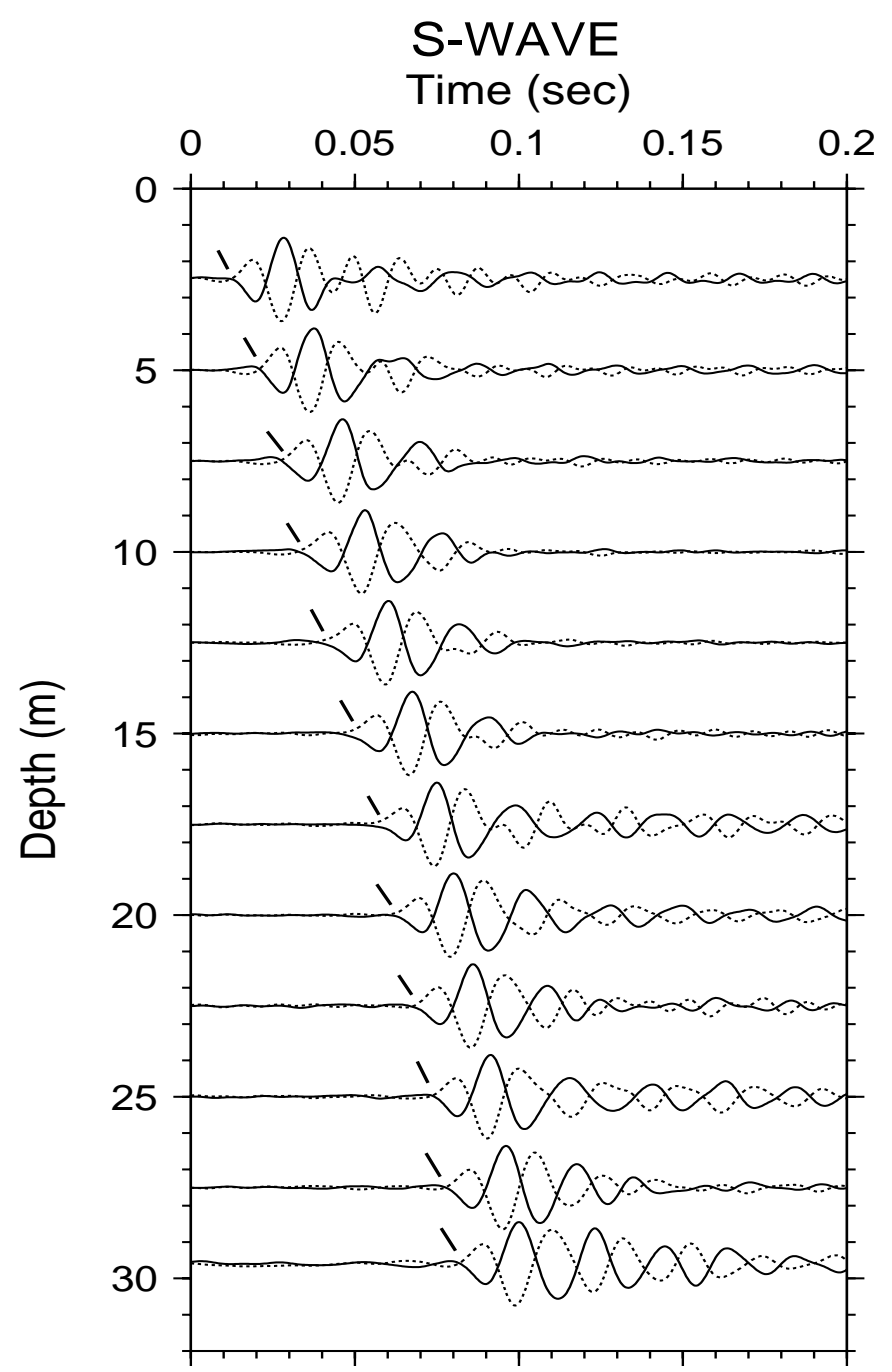

Santa Anita Golf Course

Figure A-52. Horizontal component record section (from impacts in opposite directions) superimposed for identification of S-wave onset. Approximate S-wave time picks are indicated by the hatch marks. 


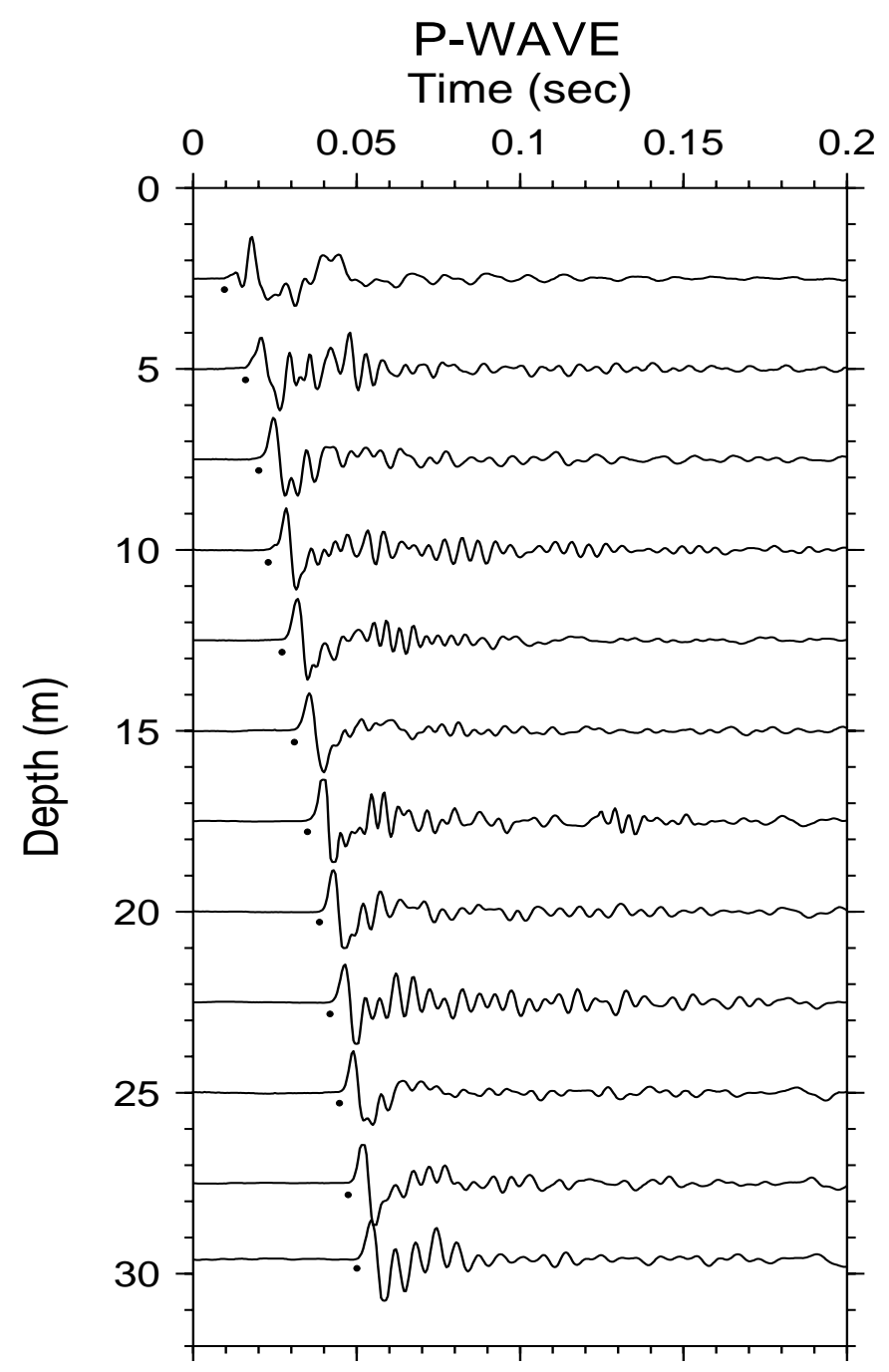

Santa Anita Golf Course

Figure A-53. Vertical component record section. Approximate P-wave arrivals are indicated by the dots. 


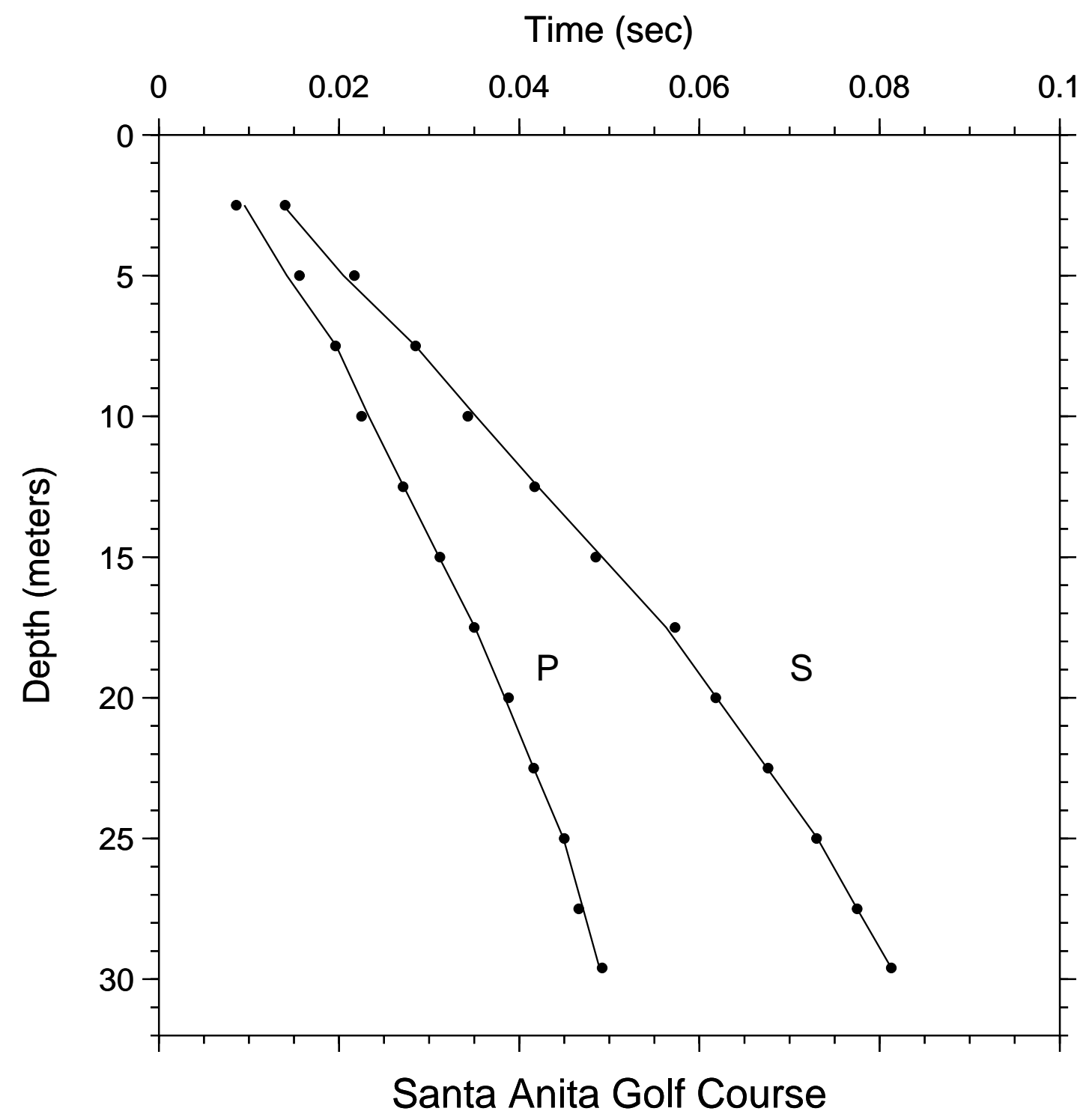

Figure A-54. Time-depth graph of P-wave and S-wave picks. Line segments are straightline interpolations of model predictions at the observation depths. The times for zero depth, not shown, are given by hoffset divided by the velocity in the uppermost layer (see accompanying tables of velocities for specific values). 


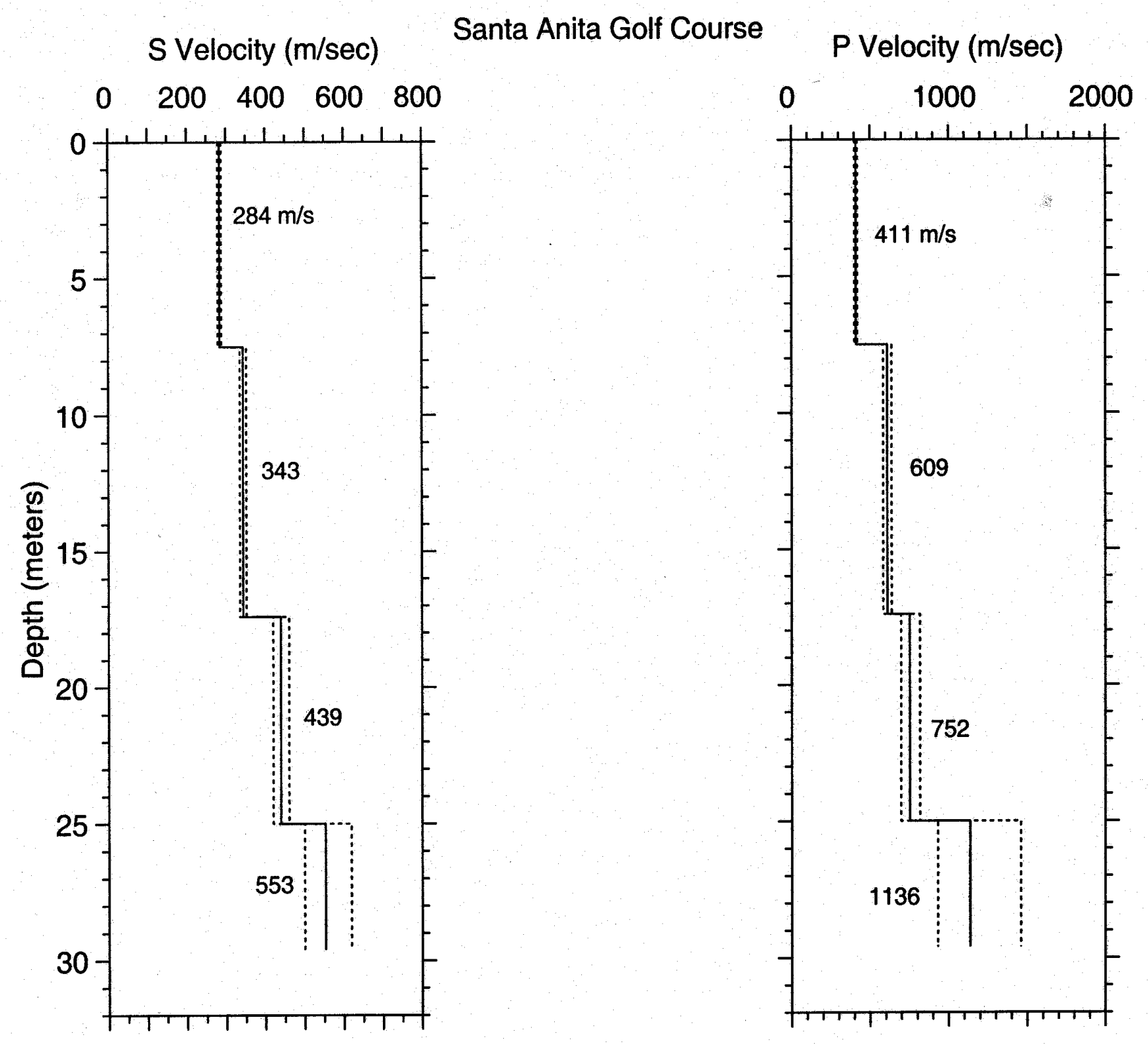

Figure A-55. S- and P-wave velocity profiles with dashed lines representing one standard deviation. Lithology is not available from this borehole. 
ABLE A-21. S-wave arrival times and velocity summaries. Location: Santa Anita Golf Course: $\$$ Coordinates:
hoffet $=\begin{aligned} 3.00 & \text { travel-time file: } P: \backslash \text { SAG SAGS_RB.TT }\end{aligned}$

$34.13096-118.03070$ Hole_Code: 304

nlayers $=4$

$\begin{array}{rrrrrrr}\mathrm{d}(\mathrm{m}) & \mathrm{d}(\mathrm{ft}) & \mathrm{tsl}(s) & \text { tvrt }(s) & \text { vavg }(\mathrm{m} / \mathrm{s}) & \text { sig } & \mathrm{rsdl}(\mathrm{sec}) \\ 2.5 & 8.2 & 0.0140 & 0.0088 & 284 & 1 & 0.0002 \\ 5.0 & 16.4 & 0.0217 & 0.0176 & 284 & 1 & 0.0012 \\ 7.5 & 24.6 & 0.0285 & 0.0264 & 284 & 1 & 0.0000 \\ 10.0 & 32.8 & 0.0343 & 0.0337 & 297 & 1 & -0.0009 \\ 12.5 & 41.0 & 0.0417 & 0.0410 & 305 & 1 & -0.0004 \\ 15.0 & 49.2 & 0.0485 & 0.0483 & 311 & 1 & -0.0007 \\ 17.5 & 57.4 & 0.0573 & 0.0555 & 315 & 1 & 0.0010 \\ 20.0 & 65.6 & 0.0618 & 0.0612 & 327 & 1 & -0.0001 \\ 22.5 & 73.8 & 0.0676 & 0.0669 & 336 & 1 & 0.0001 \\ 25.0 & 82.0 & 0.0730 & 0.0726 & 344 & 1 & -0.0001 \\ 27.5 & 90.2 & 0.0775 & 0.0771 & 357 & 1 & 0.0000 \\ 29.6 & 97.1 & 0.0813 & 0.0809 & 366 & 1 & 0.0000\end{array}$

dtb(im) thk (m) $\mathrm{v}(\mathrm{m} / \mathrm{s}) \quad \mathrm{vl}(\mathrm{m} / \mathrm{s}) \quad \mathrm{vu}(\mathrm{m} / \mathrm{s}) \quad \mathrm{dth}(\mathrm{ft}) \operatorname{thk}(\mathrm{ft}) \quad \mathrm{v}(\mathrm{ft} / \mathrm{s}) \quad \mathrm{vl}(\mathrm{ft} / \mathrm{s}) \quad \mathrm{vu}(\mathrm{ft} / \mathrm{s})$

$\begin{array}{rrrrrrrrrr}7.5 & 7.5 & 284 & 279 & 288 & 24.6 & 24.6 & 931 & 917 & 946 \\ 17.4 & 9.9 & 343 & 335 & 352 & 57.1 & 32.5 & 1126 & 1099 & 1154 \\ 25.0 & 7.6 & 439 & 420 & 460 & 82.0 & 24.9 & 1441 & 1377 & 1510 \\ 29.6 & 4.6 & 553 & 500 & 619 & 97.1 & 15.1 & 1814 & 1640 & 2031\end{array}$

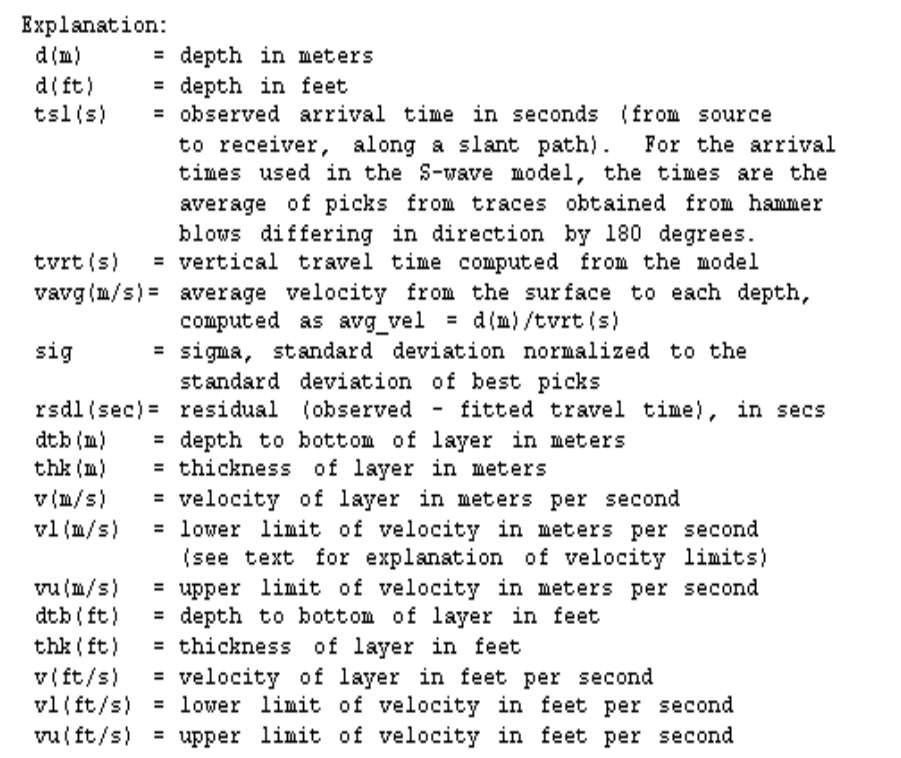


ABLE A22. P-wave arrival times and velocity sumaries. Location: Santa Anita Golf Course: P Coordinates:
hoffset $=3.00$ travel-time file: $\mathrm{F}: \backslash$ SAG $\backslash$ SAGP.TT

$\begin{array}{rrrrcrr}d(\mathbb{I}) & d(f t) & t s l(s) & \text { tvrt (s) } & \text { vavg }(\mathbb{m} / s) & \text { sig } & r s d l(s e c) \\ 2.5 & 8.2 & 0.0086 & 0.0061 & 411 & 1 & -0.0009 \\ 5.0 & 16.4 & 0.0156 & 0.0122 & 411 & 1 & 0.0014 \\ 7.5 & 24.6 & 0.0196 & 0.0182 & 411 & 1 & -0.0001 \\ 10.0 & 32.8 & 0.0225 & 0.0224 & 447 & 1 & -0.0008 \\ 12.5 & 41.0 & 0.0271 & 0.0265 & 472 & 1 & 0.0000 \\ 15.0 & 49.2 & 0.0312 & 0.0306 & 491 & 1 & 0.0001 \\ 17.5 & 57.4 & 0.0350 & 0.0346 & 505 & 1 & -0.0001 \\ 20.0 & 65.6 & 0.0388 & 0.0380 & 527 & 1 & 0.0005 \\ 22.5 & 73.8 & 0.0416 & 0.0413 & 545 & 1 & -0.0001 \\ 25.0 & 82.0 & 0.0450 & 0.0446 & 560 & 1 & 0.0001 \\ 27.5 & 90.2 & 0.0466 & 0.0468 & 587 & 1 & -0.0005 \\ 29.6 & 97.1 & 0.0492 & 0.0487 & 608 & 1 & 0.0003\end{array}$

$34.13096-118.03075$ Hole_Code: 304 nlayers $=4$

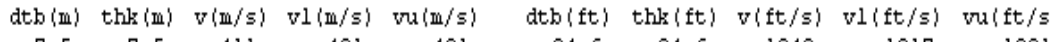

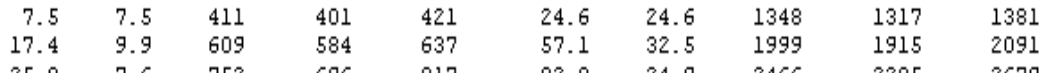

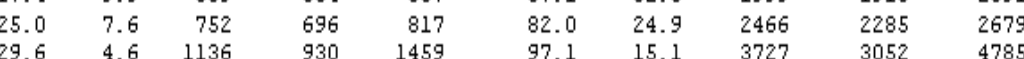

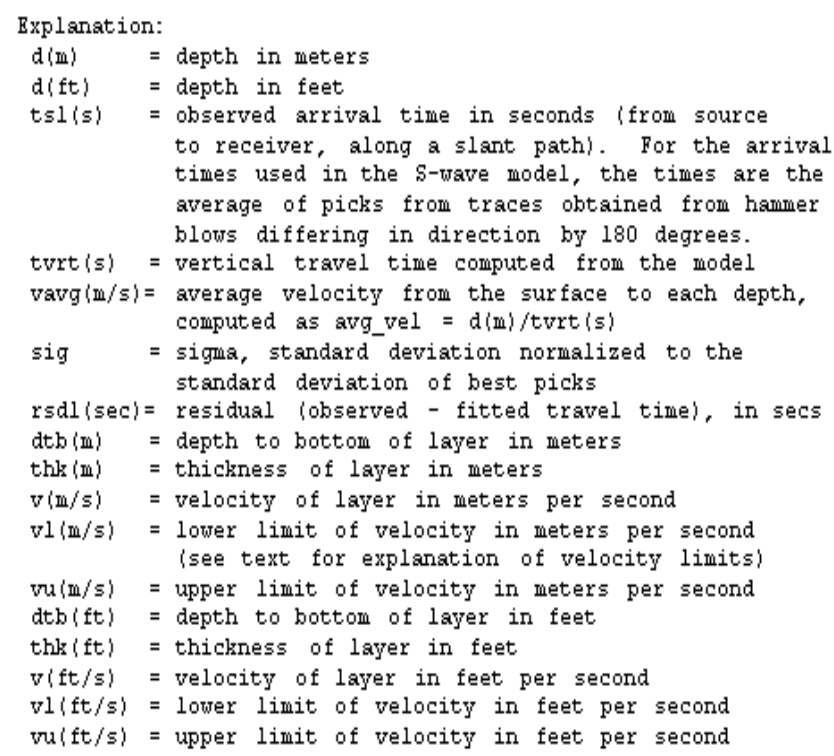






Figure A-56. Site location map for the borehole at South Western Academy. The accelerograph is located approximately 10 meters from the borehole. 


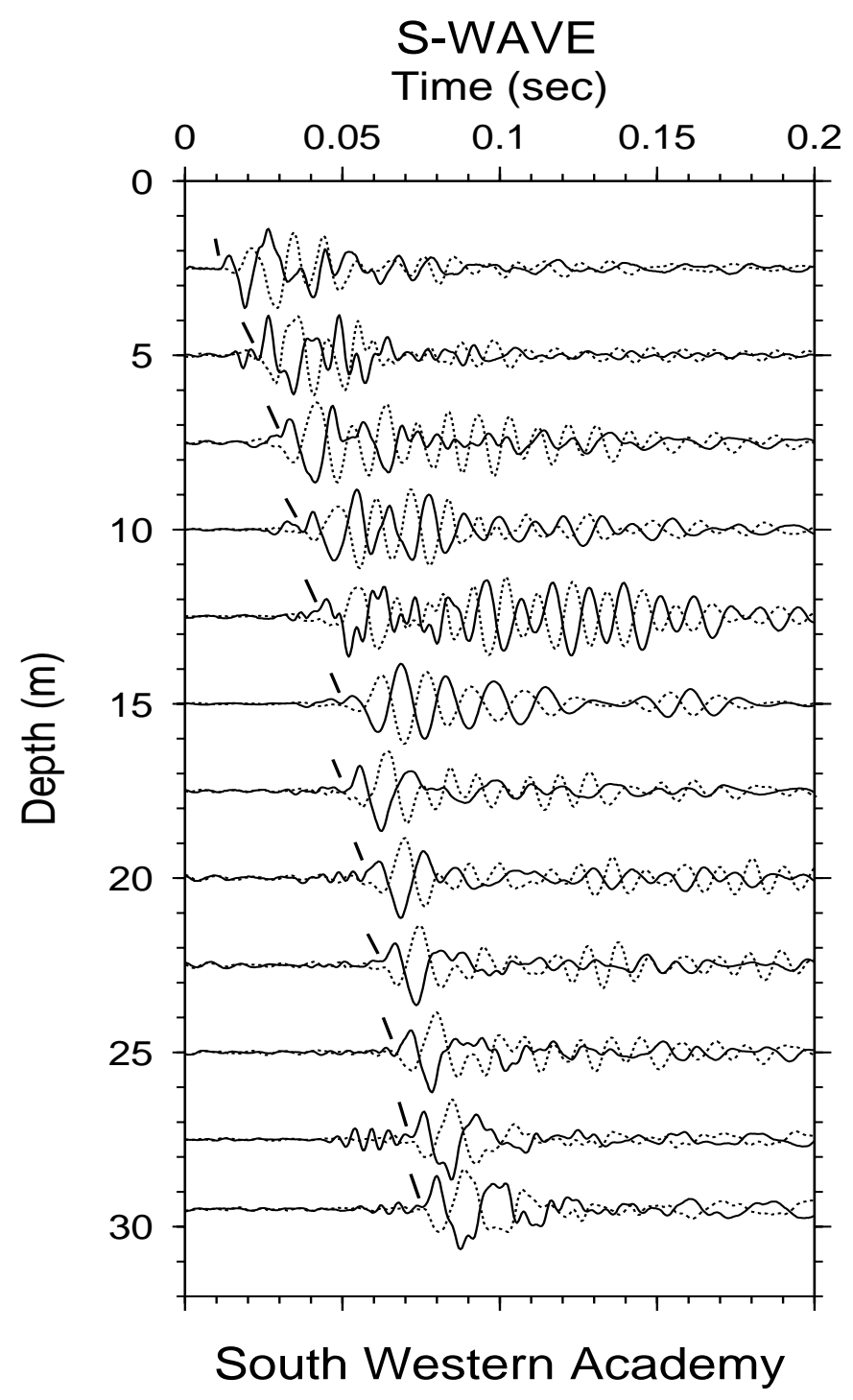

Figure A-57. Horizontal component record section (from impacts in opposite directions) superimposed for identification of S-wave onset. Approximate S-wave time picks are indicated by the hatch marks. 


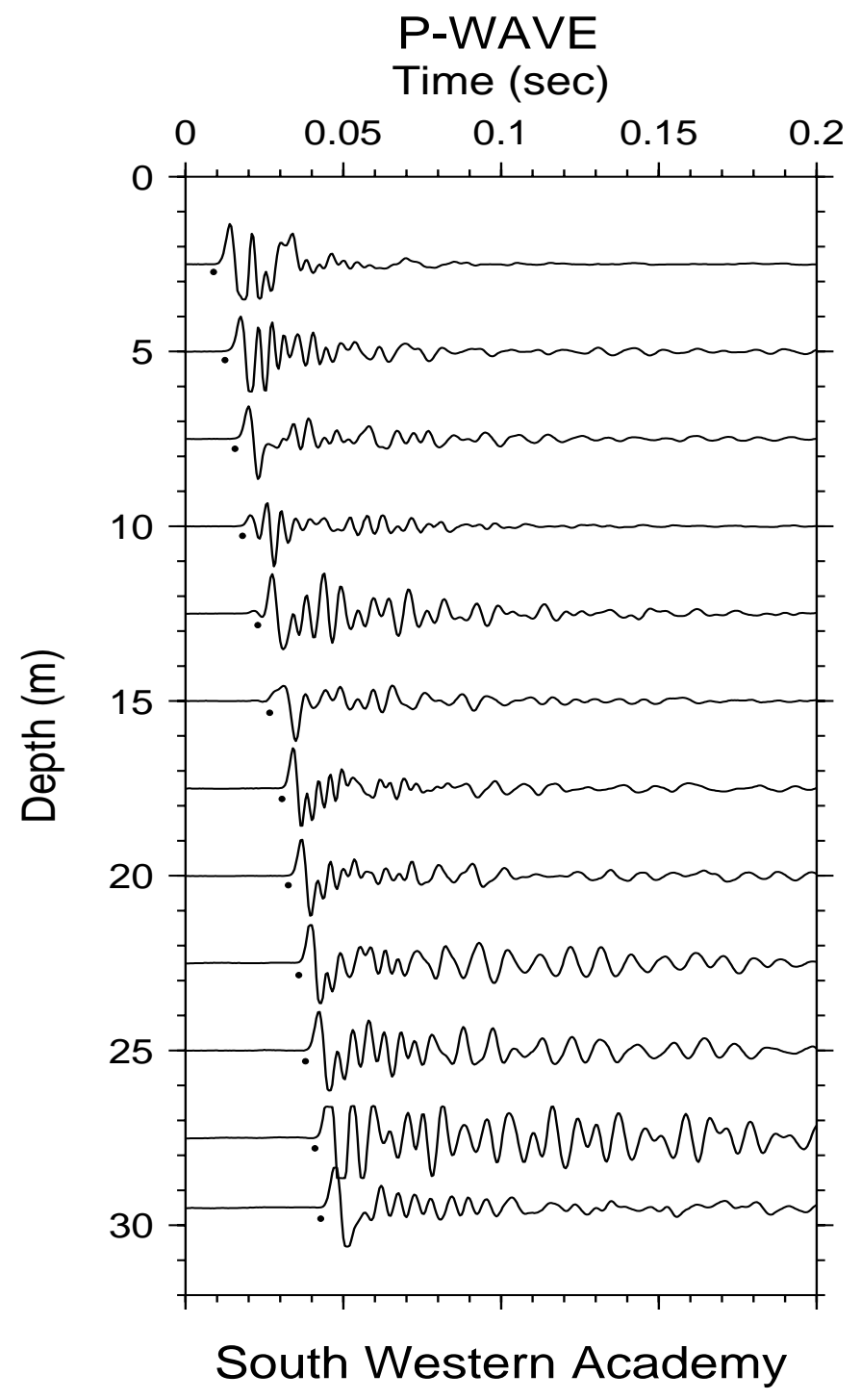

Figure A-58. Vertical component record section. Approximate P-wave arrivals are indicated by the dots. 


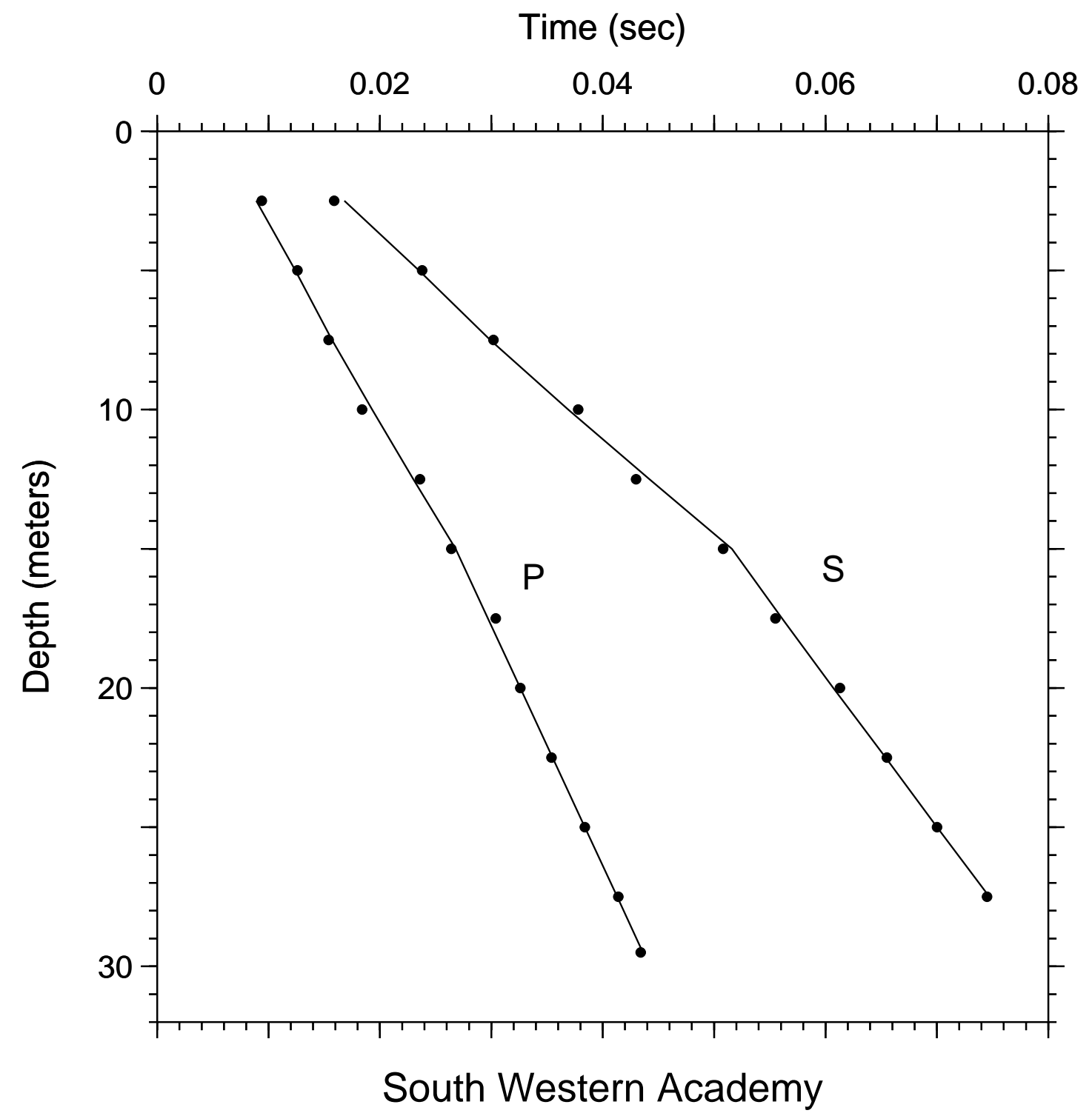

Figure A-59. Time-depth graph of P-wave and S-wave picks. Line segments are straightline interpolations of model predictions at the observation depths. The times for zero depth, not shown, are given by hoffset divided by the velocity in the uppermost layer (see accompanying tables of velocities for specific values). 


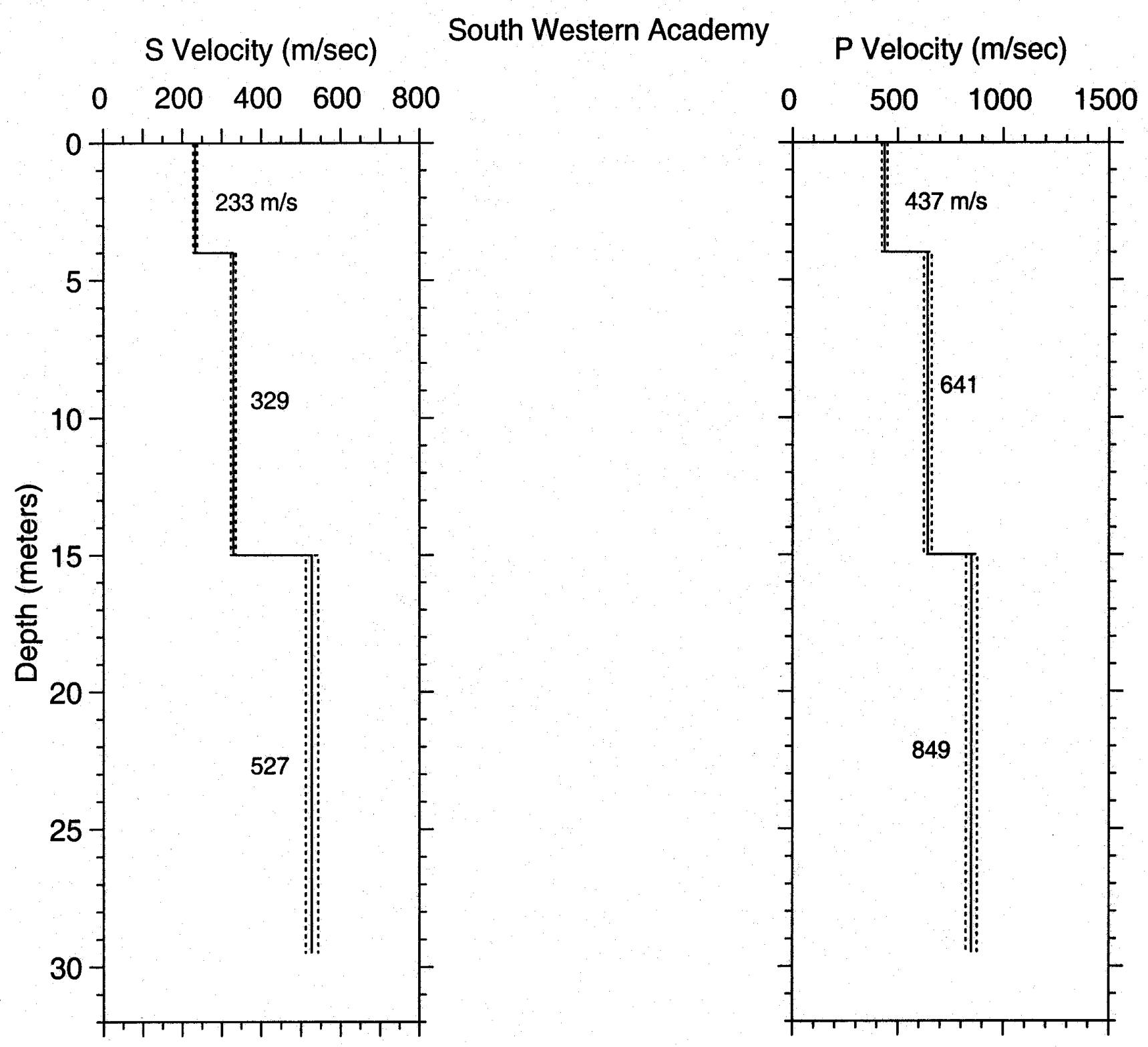

Figure A-60. S- and P-wave velocity profiles with dashed lines representing one standard deviation. Lithology is not available from this borehole. 
ABLE A23. S-wave arrival times and velocity sumaries.

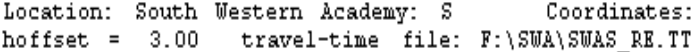

$\begin{array}{rrrrrrr}\mathrm{d}(\mathrm{m}) & \mathrm{d}(\mathrm{ft}) & \mathrm{tsl}(s) & \mathrm{twrt}(s) & \text { vavg }(\mathrm{m} / \mathrm{s}) & \text { sig } & \mathrm{rsdl}(\mathrm{sec}) \\ 2.5 & 8.2 & 0.0159 & 0.0107 & 233 & 1 & -0.0009 \\ 5.0 & 16.4 & 0.0238 & 0.0202 & 247 & 1 & 0.0003 \\ 7.5 & 24.6 & 0.0302 & 0.0278 & 270 & 1 & 0.0003 \\ 10.0 & 32.8 & 0.0378 & 0.0354 & 282 & 1 & 0.0009 \\ 12.5 & 41.0 & 0.0430 & 0.0430 & 291 & 3 & -0.0012 \\ 15.0 & 49.2 & 0.0508 & 0.0506 & 296 & 1 & -0.0008 \\ 17.5 & 57.4 & 0.0555 & 0.0553 & 316 & 2 & -0.0006 \\ 20.0 & 65.6 & 0.0613 & 0.0601 & 333 & 1 & 0.0006 \\ 22.5 & 73.8 & 0.0655 & 0.0648 & 347 & 1 & 0.0001 \\ 25.0 & 82.0 & 0.0700 & 0.0696 & 359 & 1 & 0.0000 \\ 27.5 & 90.2 & 0.0745 & 0.0743 & 370 & 1 & -0.0002\end{array}$

$34.11533-118.13050$ Hole_Code: 306 nlayers $=3$

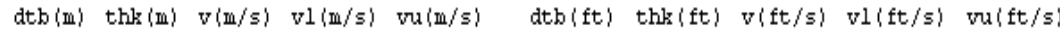

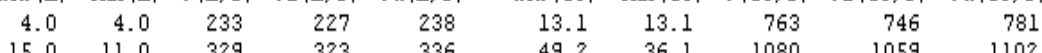

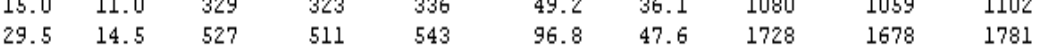

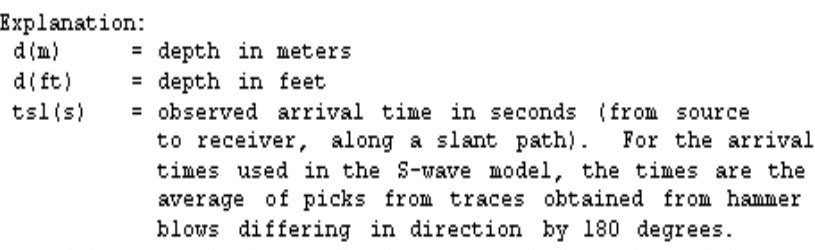


ABLE A-24. P-wave arrival times and velocity sumaries.

Location: South Western Academy: p Coordinates: hoffset $=3.00$ travel-time file: $\mathrm{P}: \backslash \mathrm{SWH} \backslash \mathrm{SWH}$. TT

$34.11533-118.13050$ Hole_Code: 306 nlayers $=3$

$\begin{array}{rrrccrr}d(\mathrm{~m}) & \mathrm{d}(\mathrm{ft}) & \mathrm{t} s l(s) & \text { tvrt }(s) & \text { vavg }(\mathrm{m} / \mathrm{s}) & \text { sig } & \mathrm{rsdl}(\mathrm{sec}) \\ 2.5 & 8.2 & 0.0094 & 0.0057 & 437 & 1 & 0.0005 \\ 5.0 & 16.4 & 0.0126 & 0.0107 & 467 & 1 & 0.0002 \\ 7.5 & 24.6 & 0.0154 & 0.0146 & 513 & 1 & -0.0003 \\ 10.0 & 32.8 & 0.0184 & 0.0185 & 540 & 1 & -0.0009 \\ 12.5 & 41.0 & 0.0236 & 0.0224 & 558 & 2 & 0.0006 \\ 15.0 & 49.2 & 0.0264 & 0.0263 & 570 & 2 & -0.0004 \\ 17.5 & 57.4 & 0.0304 & 0.0293 & 598 & 1 & 0.0007 \\ 20.0 & 65.6 & 0.0326 & 0.0322 & 621 & 1 & 0.0000 \\ 22.5 & 73.8 & 0.0354 & 0.0351 & 640 & 1 & -0.0001 \\ 25.0 & 82.0 & 0.0384 & 0.0381 & 656 & 1 & 0.0000 \\ 27.5 & 90.2 & 0.0414 & 0.0410 & 670 & 1 & 0.0001 \\ 29.5 & 96.8 & 0.0434 & 0.0434 & 680 & 1 & -0.0002\end{array}$

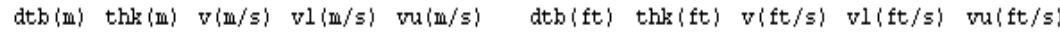
$\begin{array}{rrrrrrrrrr}4.0 & 4.0 & 437 & 423 & 451 & 13.1 & 13.1 & 1432 & 1388 & 1479\end{array}$ $\begin{array}{llllllllll}15.0 & 11.0 & 641 & 623 & 660 & 49.2 & 36.1 & 2102 & 2042 & 2165 \\ 29.5 & 14.5 & 849 & 823 & 876 & 96.8 & 47.6 & 2785 & 2701 & 2874\end{array}$

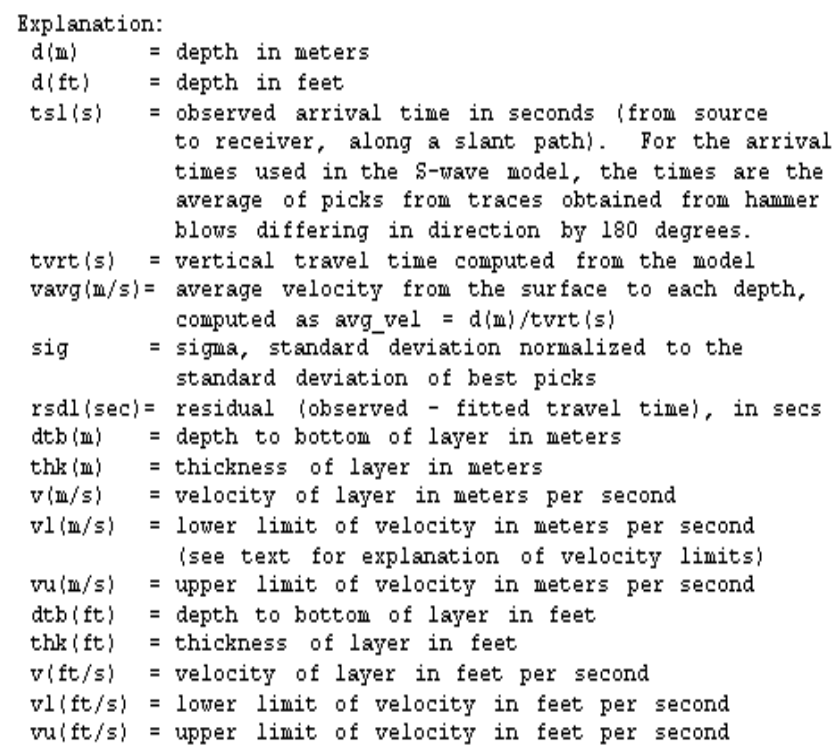






Figure A-61. Site location map for the borehole at St. Paul High School. 


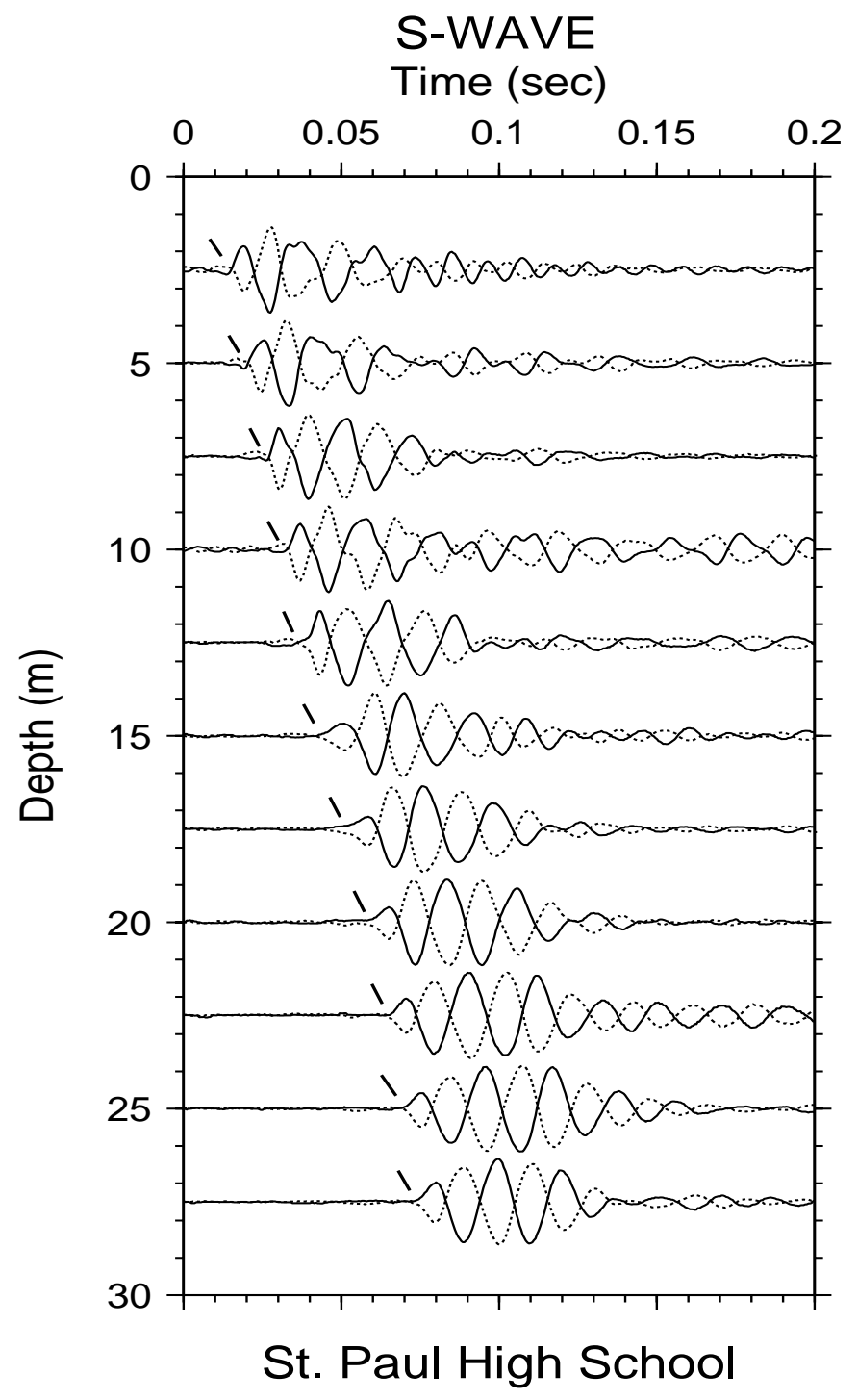

Figure A-62. Horizontal component record section (from impacts in opposite directions) superimposed for identification of S-wave onset. Approximate S-wave time picks are indicated by the hatch marks. 


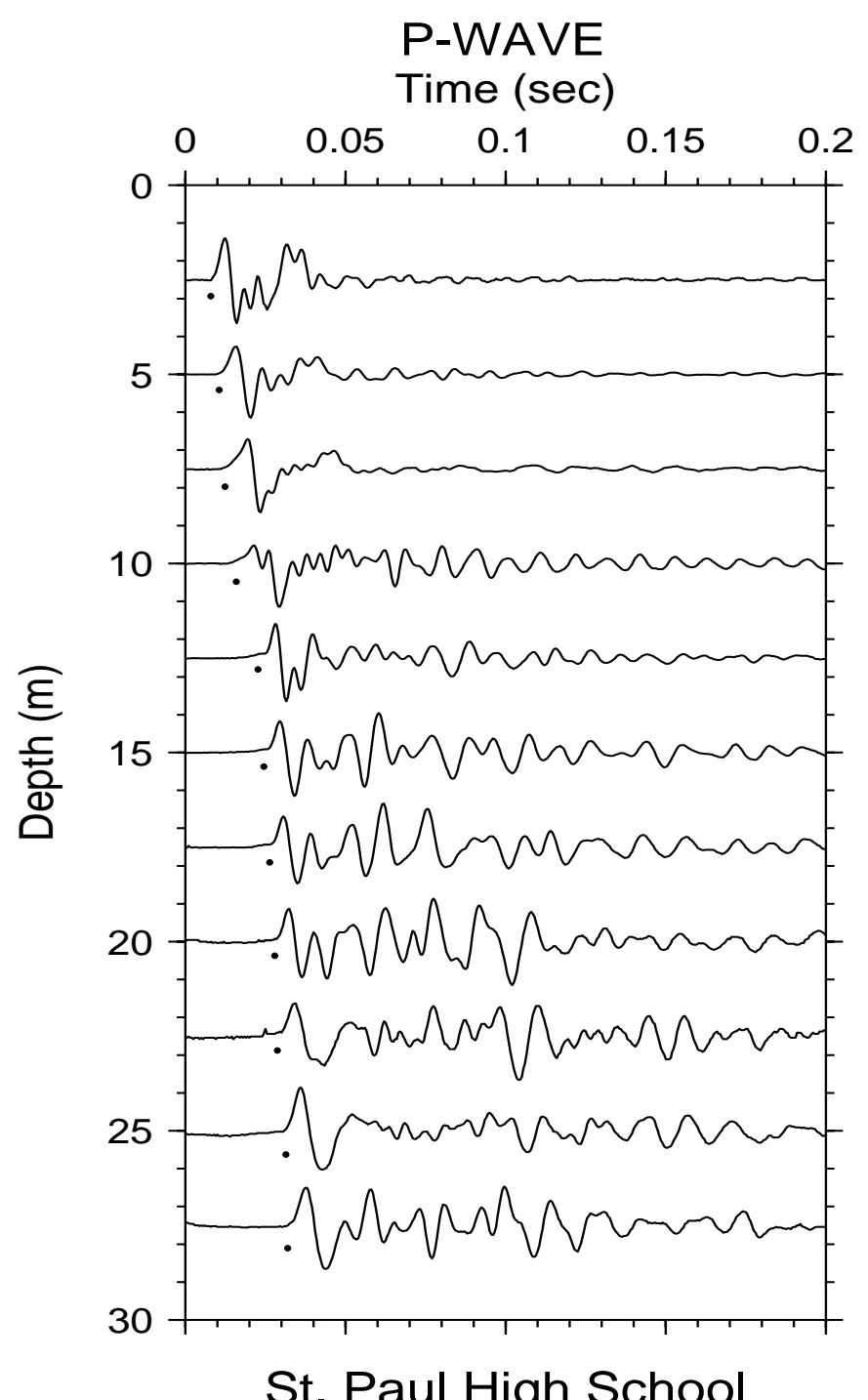

Figure A-63. Vertical component record section. Approximate P-wave arrivals are indicated by the dots. 


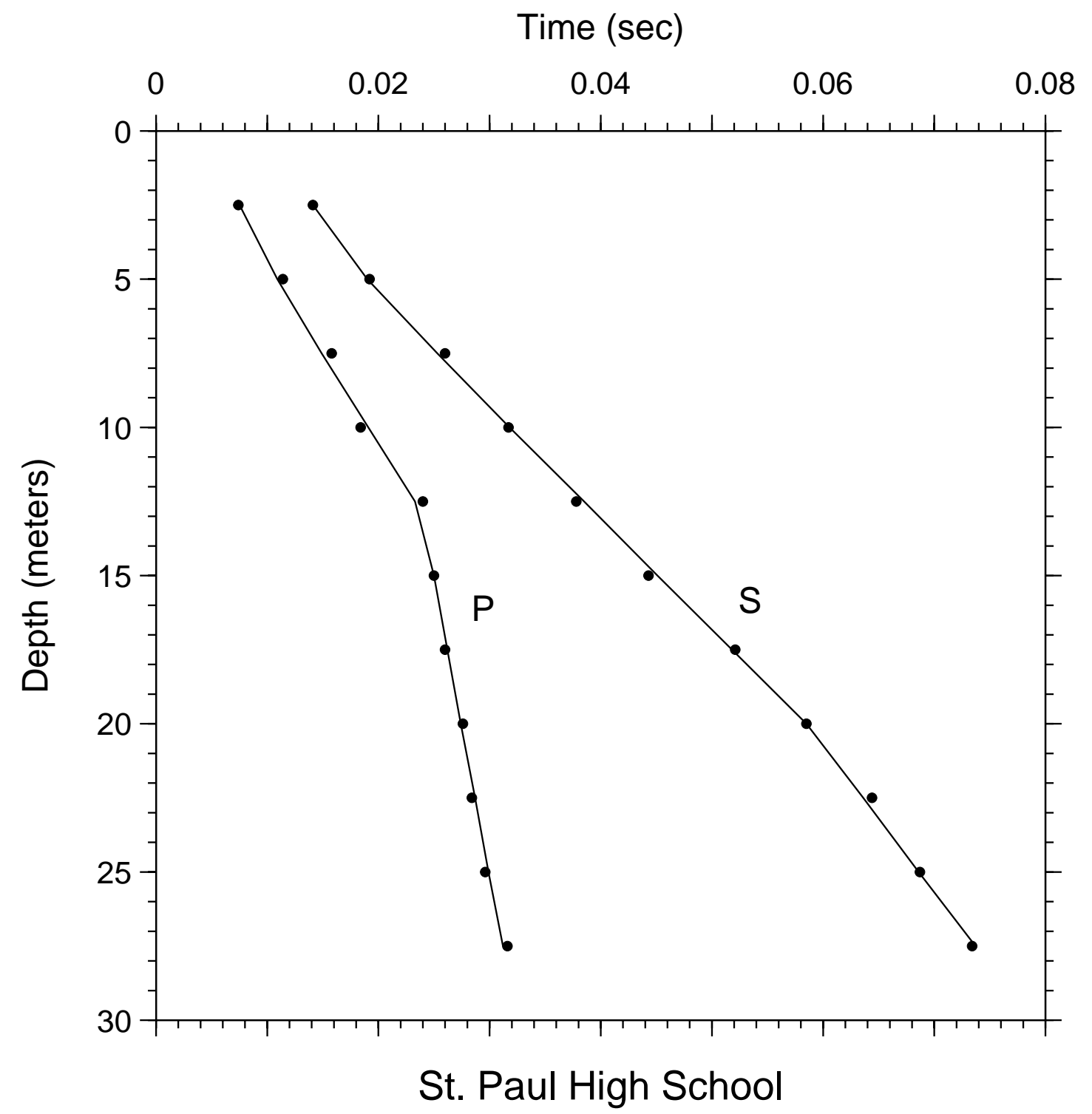

Figure A-64. Time-depth graph of P-wave and S-wave picks. Line segments are straightline interpolations of model predictions at the observation depths. The times for zero depth, not shown, are given by hoffset divided by the velocity in the uppermost layer (see accompanying tables of velocities for specific values). 


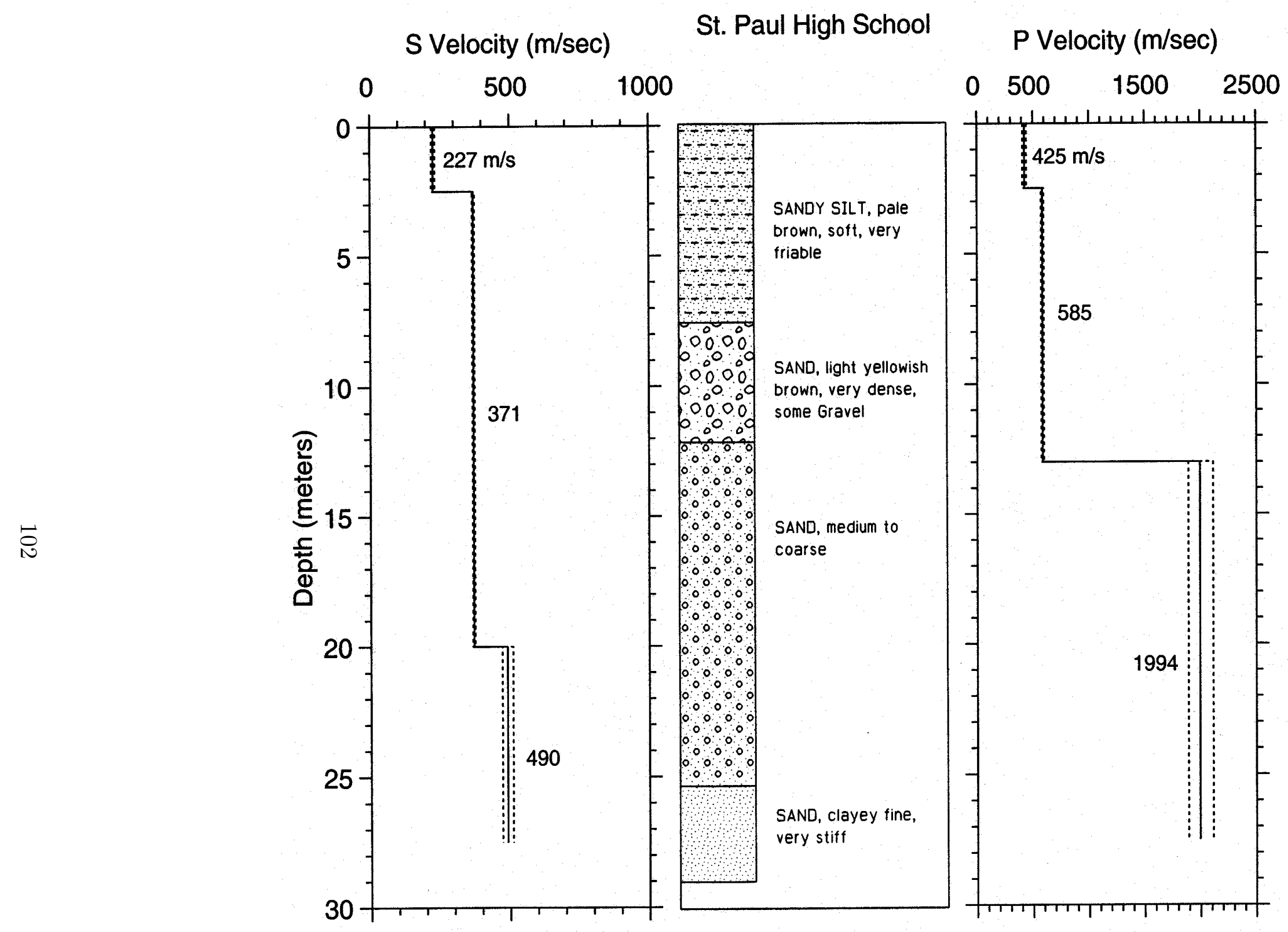

Figure A-65. S- and P-wave velocity profiles with dashed lines representing one standard deviation. Lithology is shown for correlation with velocities. 
ABLE A-25. S-wave arrival times and velocity sumaries. Location: St. Paul High School: S
hoffset $=2.00$ travel-time file:
P: $\backslash$ STP $\backslash$ STPS.TT

$\begin{array}{rrrcccr}d(\mathbb{I}) & d(f t) & t s 1(s) & \text { tvrt }(s) & \text { vavg }(\mathbb{I I} / s) & \text { sig } & \text { rsdl (sec) } \\ 2.5 & 8.2 & 0.0141 & 0.0110 & 227 & 1 & 0.0000 \\ 5.0 & 16.4 & 0.0192 & 0.0178 & 282 & 1 & 0.0001 \\ 7.5 & 24.6 & 0.0260 & 0.0245 & 306 & 1 & 0.0007 \\ 10.0 & 32.8 & 0.0317 & 0.0312 & 320 & 1 & -0.0001 \\ 12.5 & 41.0 & 0.0378 & 0.0380 & 329 & 1 & -0.0007 \\ 15.0 & 49.2 & 0.0443 & 0.0447 & 336 & 1 & -0.0008 \\ 17.5 & 57.4 & 0.0521 & 0.0514 & 340 & 1 & 0.0003 \\ 20.0 & 65.6 & 0.0585 & 0.0582 & 344 & 1 & 0.0000 \\ 22.5 & 73.8 & 0.0644 & 0.0633 & 356 & 1 & 0.0008 \\ 25.0 & 82.0 & 0.0687 & 0.0684 & 366 & 1 & 0.0001 \\ 27.5 & 90.2 & 0.0734 & 0.0735 & 374 & 1 & -0.0003\end{array}$

$33.95158-118.05369$ Hole_Code: 307 nlayers $=3$

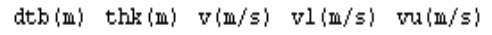

\begin{tabular}{|c|c|c|c|c|c|c|c|c|}
\hline 2.5 & 227 & 221 & 233 & 8.2 & 8.2 & 744 & 723 & 766 \\
\hline 17.5 & 371 & 367 & 375 & 65.6 & 57.4 & 1216 & 1203 & 1230 \\
\hline .5 & 990 & 471 & 510 & 90.2 & 24.6 & 1608 & 1546 & 1674 \\
\hline
\end{tabular}

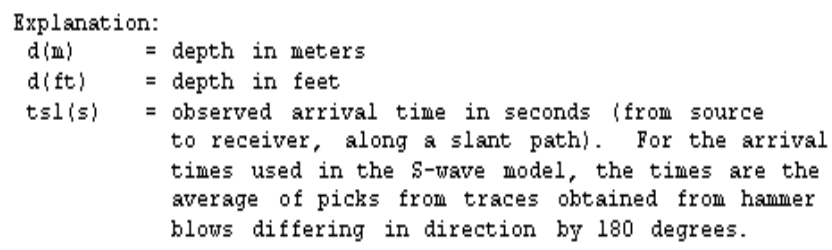


ABLE A-26. P-wave arrival times and velocity sumaries. Location: St. Paul High School: P
hoffset $=2.00$ travel-time file: $\quad$ P: $\backslash$ STP $\backslash$ STPP.TT

\begin{tabular}{|c|c|c|c|c|c|c|}
\hline (iin) & $d(\mathrm{ft})$ & $1(s)$ & $\mathrm{vr}$ & $\operatorname{vavg}(\mathbb{m} / \mathrm{s})$ & $\operatorname{sig}$ & \\
\hline 2.5 & 8.2 & 0.0074 & 0.0059 & 425 & r & -0.0001 \\
\hline 5.0 & 16.4 & 0.0114 & 0.0102 & 492 & 2 & 0.0005 \\
\hline 70 & 24.6 & 0.0158 & 0.0144 & 530 & $s$ & 0.0009 \\
\hline 0.0 & 32.8 & 0.0184 & 0.0 & 535 & 3 & -0.0007 \\
\hline .5 & 41.0 & 0.0240 & 0.0230 & 544 & 2 & 0.0007 \\
\hline & 49. & 0.0250 & 0.0 & & & \\
\hline 7.5 & 57.4 & 0.0260 & 0.0 & 67 & 1 & -0.0002 \\
\hline & 65.6 & 0.0276 & 0.0 & 7. & 1 & 0.0002 \\
\hline & 73.8 & 0.0284 & 00 & $0^{2}$ & 1 & -0.0003 \\
\hline. & 82.0 & 0.0296 & 0.0298 & 838 & 1 & -0.0003 \\
\hline & 90.2 & 0.0316 & 0.0311 & 884 & & 0.0004 \\
\hline
\end{tabular}

$33.95158-118.05369$ Hole_Code: 307 nlayers $=3$

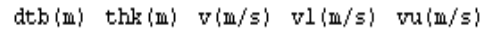

dtb $(\mathrm{ft}) \quad \mathrm{thk}(\mathrm{ft}) \quad \mathrm{v}(\mathrm{ft} / \mathrm{s}) \quad \mathrm{vl}(\mathrm{ft} / \mathrm{s}) \quad \mathrm{vu}(\mathrm{ft} / \mathrm{s})$

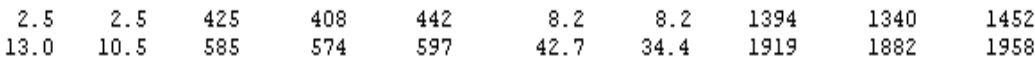

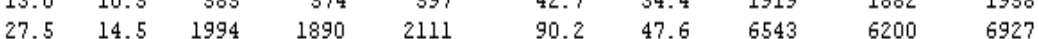

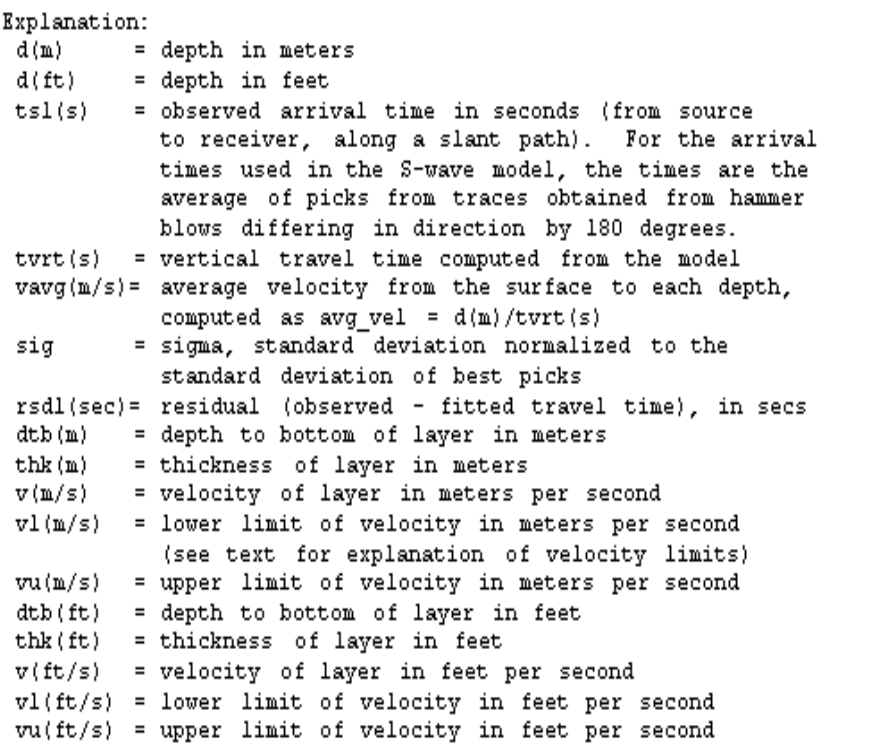


APPENDIX - B

Poisson's Ratios 
Table B-1. Poisson's ratio calculated from $\mathrm{P}$ - and $\mathrm{s}$-wave velocity models fo: the Cerritos College Gymnasium site.

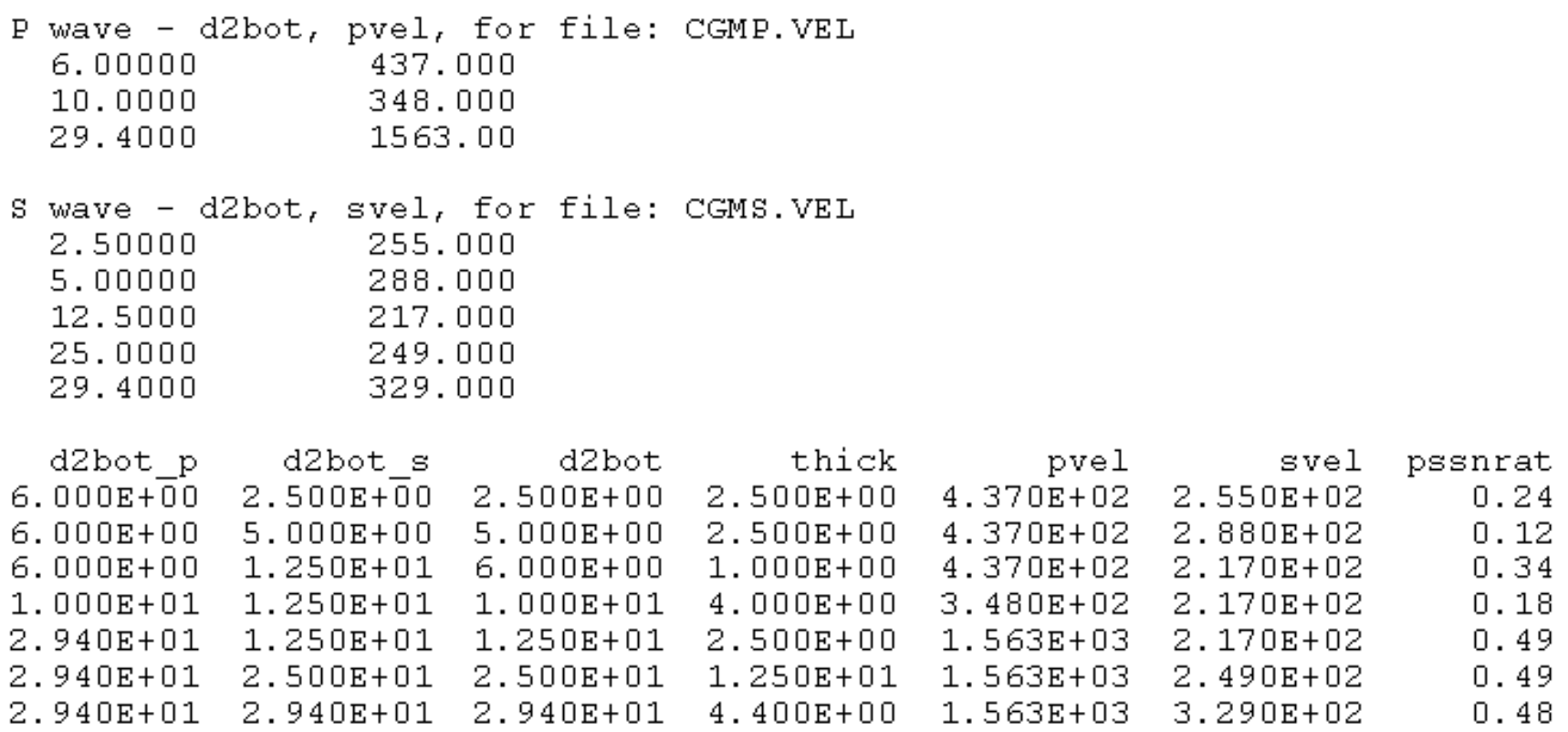


Table B-2. Poisson's ratio calculated from $\mathrm{P}-$ and $\mathrm{s}$-wave velocity models for the Cerritos College physical sciences Building site.

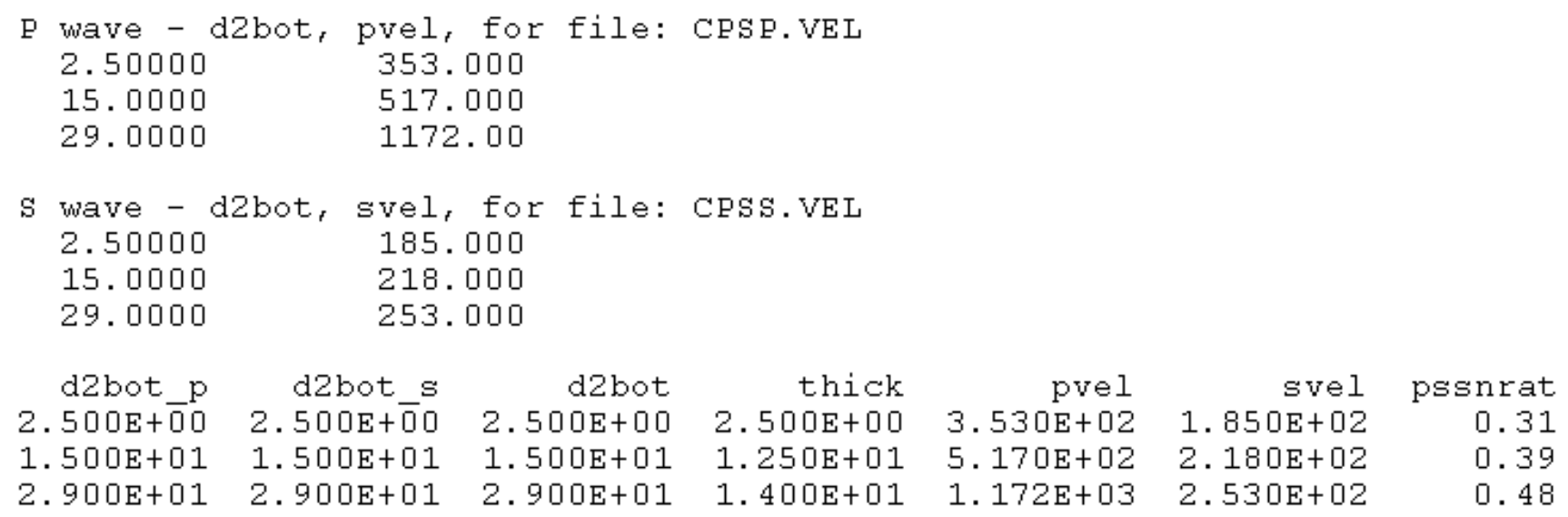


Table B-3. Poisson's ratio calculated from $\mathrm{P}-$ and $\mathrm{s}$-wave velocity models for the Cerritos College police Building site.

P wave - d2bot, pvel, for file: CPBP.VEL

$\begin{array}{ll}9.00000 & 359.000 \\ 12.5000 & 734.000 \\ 89.8000 & 1739.00\end{array}$

s wave - d2bot, svel, for file: CPBs.veL 3.00000 229.000

9.00000 204.000

23.0000 259.000

$32.0000 \quad 295.000$

46. $0000 \quad 348.000$

$78.0000 \quad 418.000$

$89.8000 \quad 450.000$

d2bot_p d2bot_s d2bot thick pvel svel pssnrat
9. $000 \mathrm{E}+\overline{0} 0$
3. $000 \mathrm{E}+\overline{0} 0$
3. $000 \mathrm{E}+00$
$3.000 \mathrm{E}+00$
$3.590 \mathrm{E}+02$
6. $000 \mathrm{E}+00$
3. $590 \mathrm{E}+02$
2. $290 \mathrm{E}+02$
0.16
9. $000 \mathrm{E}+00$
9. $000 \mathrm{E}+00$
9. $000 \mathrm{E}+00$
7. $340 \mathrm{E}+02$
2. $040 \mathrm{E}+02$
0.26
1. $250 \mathrm{E}+01$
2. $300 \mathrm{E}+01$
1. $250 \mathrm{E}+01$
3. $500 \mathrm{E}+00$
2. $590 \mathrm{E}+02$
0. 43
8. $980 \mathrm{E}+01$
2. $300 E+01$
2. $300 \mathrm{E}+01$
1. $050 \mathrm{E}+01$
1. $739 \mathrm{E}+03$
2. $590 \mathrm{E}+02$
9. $000 \mathrm{E}+00$
1. $739 \mathrm{E}+03$
2. $950 \mathrm{E}+02$
0.49
8. $980 \mathrm{E}+01$
4. $600 \mathrm{E}+01$
$3.200 E+01$
1. $400 \mathrm{E}+01$
1. $739 \mathrm{E}+03$
3. $480 \mathrm{E}+02$
0.49
7. $800 \mathrm{E}+01$
3. $200 E+01$
1. $739 \mathrm{E}+03$
4. $180 \mathrm{E}+02$
0.48
8. $980 \mathrm{E}+01$
8. $980 \mathrm{E}+01$
8. $980 \mathrm{E}+01$
1. $180 \mathrm{E}+01$
1. $739 \mathrm{E}+03$
4. $500 \mathrm{E}+02$
0. 47
0. 46 
Table B-4. Poisson's ratio calculated from the $\mathrm{P}-$ and $\mathrm{s}$-wave velocity models for the Corps of Engineer's site.

P wave - d2bot, pvel, for file: NARP.VEL

$6.00000 \quad 362.000$

$12.0000 \quad 798.000$

$22.0000 \quad 1409.00$

S wave - d2bot, svel, for file: NARS.VEL

$6.00000 \quad 241.000$

$12.0000 \quad 212.000$

$22.0000 \quad 381.000$

$\begin{array}{rrrrrrr}\text { d2botp } & \text { d2bot s } & \text { d2bot } & \text { thick } & \text { pvel } & \text { svel } & \text { pssnrat } \\ 6.000 \mathrm{E}+00 & 6.000 \mathrm{E}+00 & 6.000 \mathrm{E}+00 & 6.000 \mathrm{E}+00 & 3.620 \mathrm{E}+02 & 2.410 \mathrm{E}+02 & 0.10 \\ 1.200 \mathrm{E}+01 & 1.200 \mathrm{E}+01 & 1.200 \mathrm{E}+01 & 6.000 \mathrm{E}+00 & 7.980 \mathrm{E}+02 & 2.120 \mathrm{E}+02 & 0.46 \\ 2.200 \mathrm{E}+01 & 2.200 \mathrm{E}+01 & 2.200 \mathrm{E}+01 & 1.000 \mathrm{E}+01 & 1.409 \mathrm{E}+03 & 3.810 \mathrm{E}+02 & 0.46\end{array}$


Table B-5. Poisson's ratio calculated from $\mathrm{P}-$ and $\mathrm{s}$-wave velocity models for the Hoover school site.

$P$ wave - d2bot, pvel, for file: HOOP2.VEL

$7.50000 \quad 680.000$

$25.0000 \quad 1283.00$

s wave - d2bot, svel, for file: Hoos2. VEL

$7.50000 \quad 470.000$

$25.0000 \quad 790.000$

d2bot_p d2bot_s d2bot thick pvel svel pssnrat

$\begin{array}{llllllll}7.500 \mathrm{E}+\overline{0} 0 & 7.500 \mathrm{E}+\overline{0} 0 & 7.500 \mathrm{E}+00 & 7.500 \mathrm{E}+00 & 6.800 \mathrm{E}+02 & 4.700 \mathrm{E}+02 & 0.04\end{array}$

$\begin{array}{lllllll}2.500 \mathrm{E}+01 & 2.500 \mathrm{E}+01 & 2.500 \mathrm{E}+01 & 1.750 \mathrm{E}+01 & 1.283 \mathrm{E}+03 & 7.900 \mathrm{E}+02 & 0.19\end{array}$ 
Table B-6. Poisson's ratio calculated from $\mathrm{P}-$ and $\mathrm{S}$-wave velocity models for the Lincoln school site.

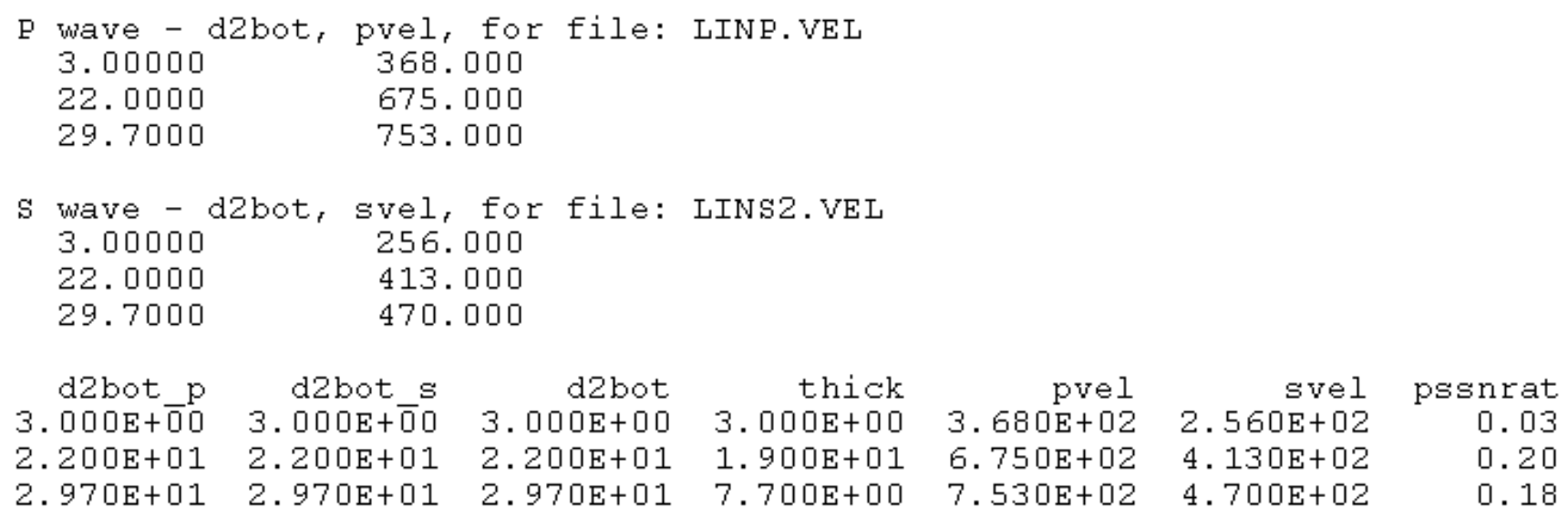


Table B-7. Poisson's ratio calculated from $\mathrm{P}$ - and s-wave velocity models for the Lincoln school whittier site.

P wave - d2bot, pvel, for file: wLBVERT.VEL

$1.50000 \quad 224.000$

$7.50000 \quad 1382.00$

$18.5000 \quad 517.000$

S wave - d2bot, svel, for file:

$1.50000 \quad 139.000$

$18.5000 \quad 347.000$

d2bot_p d2bot_s d2bot thick pvel svel pssnrat

$\begin{array}{lllllll}1.500 \mathrm{E}+\overline{0} 0 & 1.500 \mathrm{E}+\overline{0} 0 & 1.500 \mathrm{E}+00 & 1.500 \mathrm{E}+00 & 2.240 \mathrm{E}+02 & 1.390 \mathrm{E}+02 & 0.19\end{array}$

$\begin{array}{lllllll}7.500 \mathrm{E}+00 & 1.850 \mathrm{E}+01 & 7.500 \mathrm{E}+00 & 6.000 \mathrm{E}+00 & 1.382 \mathrm{E}+03 & 3.470 \mathrm{E}+02 & 0.47\end{array}$

$\begin{array}{lllllll}1.850 \mathrm{E}+01 & 1.850 \mathrm{E}+01 & 1.850 \mathrm{E}+01 & 1.100 \mathrm{E}+01 & 5.170 \mathrm{E}+02 & 3.470 \mathrm{E}+02 & 0.09\end{array}$ 
Table B-8. Poisson's ratio calculated from $\mathrm{P}-$ and $\mathrm{s}$-wave velocity models for the Los Alisos Adult school site.

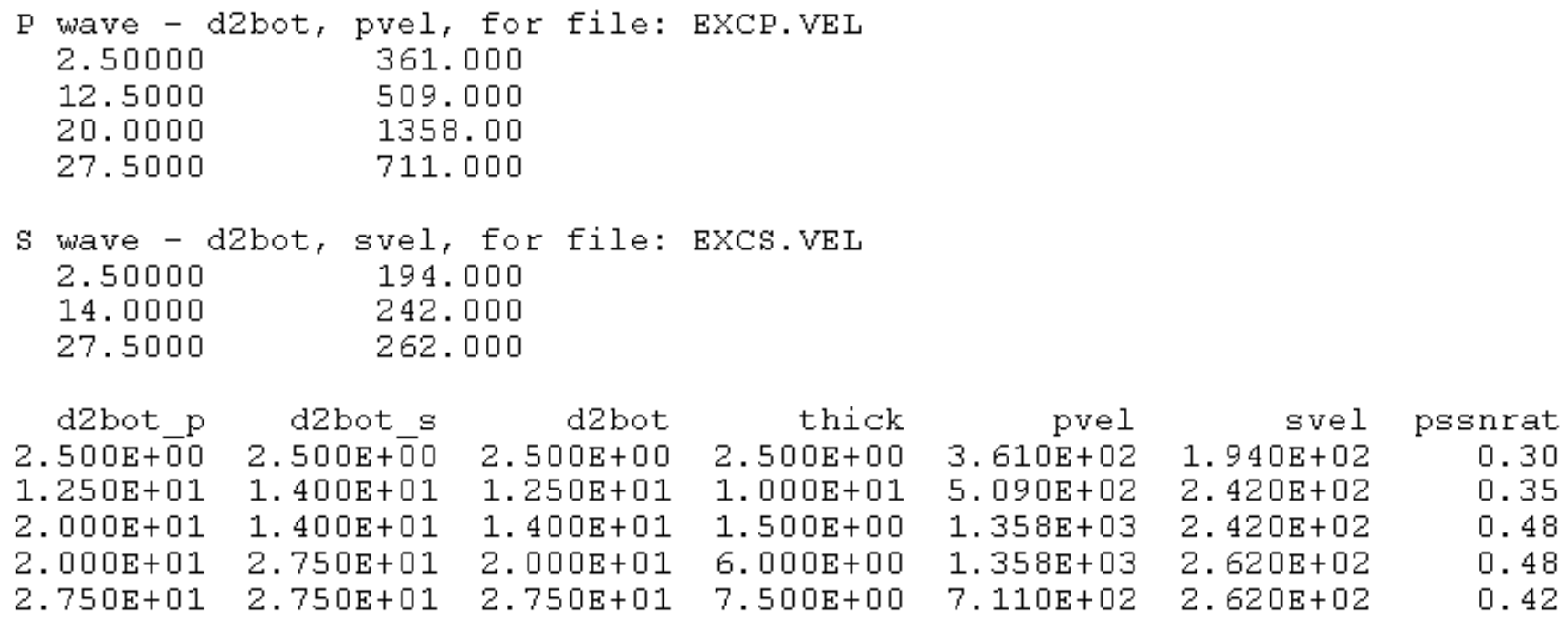


Table B-9. Poisson's ratio calculated from $\mathrm{P}-$ and $\mathrm{s}$-wave velocity models for the olive Junior High school site.

P wave - d2bot, pvel, for file: OLVP.VEL

$1.50000 \quad 309.000$

$11.0000 \quad 786.000$

$16.8000 \quad 983.000$

s wave - d2bot, svel, for file: OLVS.VEL

$1.50000 \quad 209.000$

$11.0000 \quad 527.000$

$16.8000 \quad 636.000$

d2bot p d2bot $s$ d2bot thick pvel svel pssnrat

$\begin{array}{lllllll}1.500 \mathrm{E}+\overline{0} 0 & 1.500 \mathrm{E}+\overline{0} 0 & 1.500 \mathrm{E}+00 & 1.500 \mathrm{E}+00 & 3.090 \mathrm{E}+02 & 2.090 \mathrm{E}+02 & 0.08\end{array}$

$\begin{array}{lllllll}1.100 \mathrm{E}+01 & 1.100 \mathrm{E}+01 & 1.100 \mathrm{E}+01 & 9.500 \mathrm{E}+00 & 7.860 \mathrm{E}+02 & 5.270 \mathrm{E}+02 & 0.09\end{array}$

$\begin{array}{lllllll}1.680 \mathrm{E}+01 & 1.680 \mathrm{E}+01 & 1.680 \mathrm{E}+01 & 5.800 \mathrm{E}+00 & 9.830 \mathrm{E}+02 & 6.360 \mathrm{E}+02 & 0.14\end{array}$ 
Table B-10. Poisson's ratio calculated from $\mathrm{P}$ - and s-wave velocity models for the San Bernardino Fire station site.

P wave - d2bot, pvel, for file: sBlp.VEL

$8.50000 \quad 430.000$

$16.2000 \quad 936.000$

$70.0000 \quad 1647.00$

$90.0000 \quad 2094.00$

S wave - d2bot, svel, for file: sBls.veL

$3.70000 \quad 297.000$

$16.2000 \quad 299.000$

$41.2000 \quad 365.000$

$59.5000 \quad 408.000$

$78.0000 \quad 460.000$

$90.0000 \quad 556.000$

d2bot_p d2bot_s d2bot thick pvel svel pssnrat

$\begin{array}{lllllll}8.500 \mathrm{E}+00 & 3.700 \mathrm{E}+00 & 3.700 \mathrm{E}+00 & 3.700 \mathrm{E}+00 & 4.300 \mathrm{E}+02 & 2.970 \mathrm{E}+02 & 0.04 \\ 8.500 \mathrm{E}+00 & 1.620 \mathrm{E}+01 & 8.500 \mathrm{E}+00 & 4.800 \mathrm{E}+00 & 4.300 \mathrm{E}+02 & 2.990 \mathrm{E}+02 & 0.03 \\ 1.620 \mathrm{E}+01 & 1.620 \mathrm{E}+01 & 1.620 \mathrm{E}+01 & 7.700 \mathrm{E}+00 & 9.360 \mathrm{E}+02 & 2.990 \mathrm{E}+02 & 0.44 \\ 7.000 \mathrm{E}+01 & 4.120 \mathrm{E}+01 & 4.120 \mathrm{E}+01 & 2.500 \mathrm{E}+01 & 1.647 \mathrm{E}+03 & 3.650 \mathrm{E}+02 & 0.47 \\ 7.000 \mathrm{E}+01 & 5.950 \mathrm{E}+01 & 5.950 \mathrm{E}+01 & 1.830 \mathrm{E}+01 & 1.647 \mathrm{E}+03 & 4.080 \mathrm{E}+02 & 0.47 \\ 7.000 \mathrm{E}+01 & 7.800 \mathrm{E}+01 & 7.000 \mathrm{E}+01 & 1.050 \mathrm{E}+01 & 1.647 \mathrm{E}+03 & 4.600 \mathrm{E}+02 & 0.46 \\ 9.000 \mathrm{E}+01 & 7.800 \mathrm{E}+01 & 7.800 \mathrm{E}+01 & 8.000 \mathrm{E}+00 & 2.094 \mathrm{E}+03 & 4.600 \mathrm{E}+02 & 0.47 \\ 9.000 \mathrm{E}+01 & 9.000 \mathrm{E}+01 & 9.000 \mathrm{E}+01 & 1.200 \mathrm{E}+01 & 2.094 \mathrm{E}+03 & 5.560 \mathrm{E}+02 & 0.46\end{array}$


Table B-11. Poisson's ratio calculated from the $\mathrm{p}-$ and $\mathrm{s}$-wave velocity model for the santa Anita Golf Course site.

\begin{tabular}{|c|c|c|c|c|c|c|}
\hline $\begin{array}{l}\text { wave - } \\
7.50000 \\
17.4000 \\
25.0000 \\
29.6000\end{array}$ & $\begin{array}{r}\text { d2bot, pvel, } \\
411 . \\
609 . \\
752 . \\
1136\end{array}$ & $\begin{array}{l}\text { for } \\
000 \\
000 \\
000 \\
00\end{array}$ & & & & \\
\hline $\begin{array}{l}\text { S wave - } \\
7.50000 \\
17.4000 \\
25.0000 \\
29.6000\end{array}$ & $\begin{array}{r}\text { d2bot, svel, } \\
284 . \\
343 . \\
439 . \\
553 .\end{array}$ & $\begin{array}{l}\text { for file: } \\
000 \\
000 \\
000 \\
000\end{array}$ & SAGS_RE . VEL & & & \\
\hline $\begin{array}{r}\text { d2bot_p } \\
7.500 \mathrm{E}+00 \\
1.740 \mathrm{E}+01 \\
2.500 \mathrm{E}+01 \\
2.960 \mathrm{E}+01\end{array}$ & $\begin{array}{r}\text { d2bot_s } \\
7.500 \mathrm{E}+00 \\
1.740 \mathrm{E}+01 \\
2.500 \mathrm{E}+01 \\
2.960 \mathrm{E}+01\end{array}$ & $\begin{array}{r}\mathrm{d} 2 \mathrm{bot} \\
7.500 \mathrm{E}+00 \\
1.740 \mathrm{E}+01 \\
2.500 \mathrm{E}+01 \\
2.960 \mathrm{E}+01\end{array}$ & $\begin{array}{r}\text { thick } \\
7.500 \mathrm{E}+00 \\
9.900 \mathrm{E}+00 \\
7.600 \mathrm{E}+00 \\
4.600 \mathrm{E}+00\end{array}$ & $\begin{array}{r}\text { pvel } \\
4.110 \mathrm{E}+02 \\
6.090 \mathrm{E}+02 \\
7.520 \mathrm{E}+02 \\
1.136 \mathrm{E}+03\end{array}$ & $\begin{array}{r}\text { svel } \\
2.840 \mathrm{E}+02 \\
3.430 \mathrm{E}+02 \\
4.390 \mathrm{E}+02 \\
5.530 \mathrm{E}+02\end{array}$ & $\begin{array}{r}\text { pssnrat } \\
0.04 \\
0.27 \\
0.24 \\
0.34\end{array}$ \\
\hline
\end{tabular}


Table B-12. Poisson's ratio calculated from $\mathrm{P}$ - and $\mathrm{s}$-wave velocity models for the South Wiestern Academy site.

P wave - d2bot, pvel, for file: SWAP.VEL 4.00000 437.000

$15.0000 \quad 641.000$

$29.5000 \quad 849.000$

S wave - d2bot, svel, for file: STIS RE.VEL

4.00000 233.000

$15.0000 \quad 329.000$

$29.5000 \quad 527.000$

d2bot_p d2bot_s d2bot thick pvel svel pssnrat $\begin{array}{lllllll}4.000 \mathrm{E}+\overline{0} 0 & 4.000 \mathrm{E}+\overline{0} 0 & 4.000 \mathrm{E}+00 & 4.000 \mathrm{E}+00 & 4.370 \mathrm{E}+02 & 2.330 \mathrm{E}+02 & 0.30\end{array}$

$\begin{array}{lllllll}1.500 \mathrm{E}+01 & 1.500 \mathrm{E}+01 & 1.500 \mathrm{E}+01 & 1.100 \mathrm{E}+01 & 6.410 \mathrm{E}+02 & 3.290 \mathrm{E}+02 & 0.32\end{array}$

$\begin{array}{lllllll}2.950 \mathrm{E}+01 & 2.950 \mathrm{E}+01 & 2.950 \mathrm{E}+01 & 1.450 \mathrm{E}+01 & 8.490 \mathrm{E}+02 & 5.270 \mathrm{E}+02 & 0.19\end{array}$ 
Table B-13. Poisson's ratio calculated from $\mathrm{P}$ - and $\mathrm{s}$-wave velocity models for the st. Paul High school site.

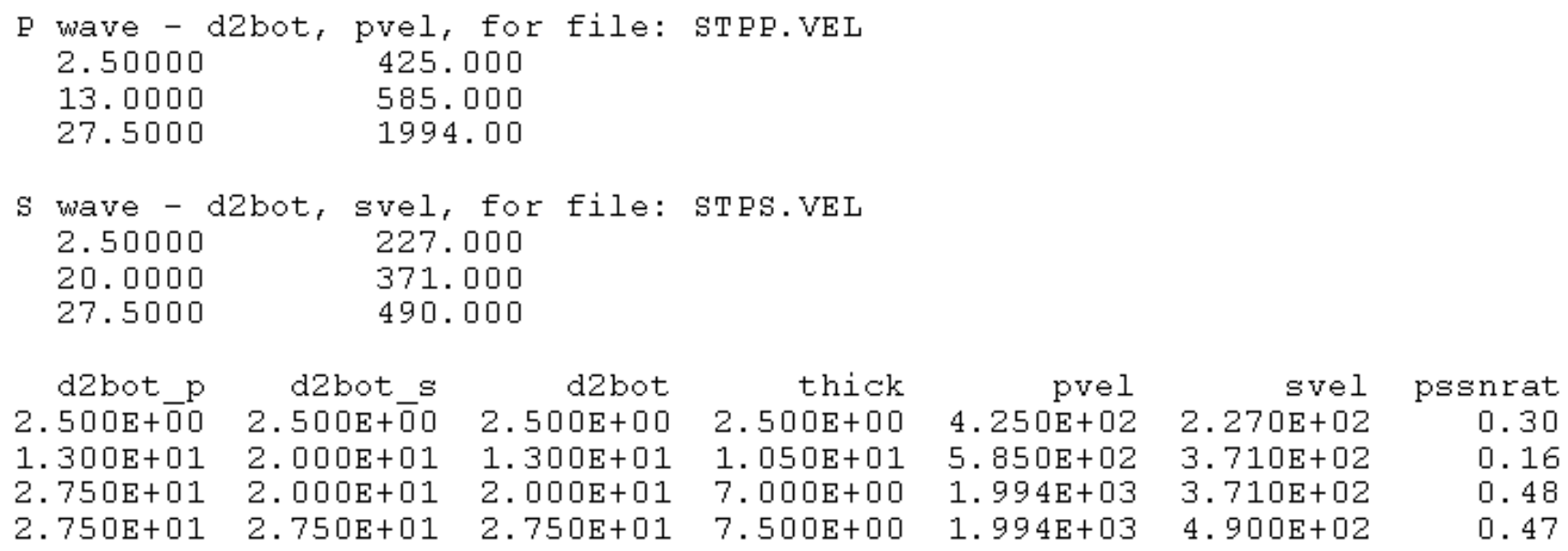

